$\sin =\sin$

NASA CR-137888

Available to the Public

NUMERICAL STUDIES OF THREE-DIMENSIONAL

BREAKDOWN IN TRAILING VORTEX WAKES

(NASA-CR-137888) = NOUERICAI STUDIES OF :

N77-10014.

THREE-DIUENSIONAL BREAKDOWN. IN TRAIIING

VORTEX WAKES: (Lockheed-Georgias Co...

Marietta.), $100=\mathrm{p}$ HC A05/MF AOF, CSCI $20 \mathrm{D}$

Unclas

by

P. F. Evans

J. E. Hackett

JUNE 1976

Distribution of this report is provided in the interest of information exchange. Responsibility for the contents resides in the authors or organization that prepared it.

Prepared under Contract No. NAS2-8651 by

LOCKHEED-GEORGIA COMPANY

Marietta, Georgia

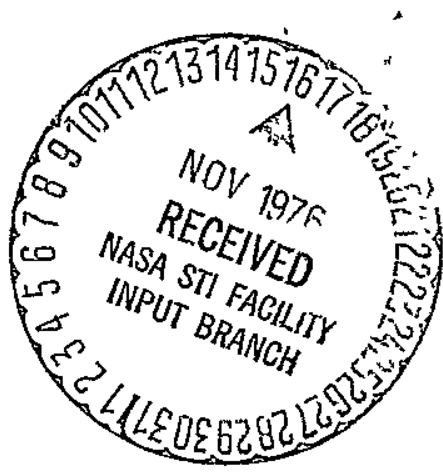

for Ames Research Center

NATIONAL AERONAUTICS AND SPACE ADMINISTRATION 
NASA CR-137888

Available to the Public

NUMERICAL STUDIES OF THREE-DIMENSIONAL

BREAKDOWN IN TRAILING VORTEX WAKES

by

P. F. Evans

J. E. Hackett

JUNE 1976

Distribution of this report is provided in the interest of information exchange. Responsibility for the contents resides in the authors or organization that prepared it.

Prepared under Contract No. NAS2-8651 by

LOCKHEED-GEORGIA COMPANY

Marietta, Georgia

for Ames Research Center

NATIONAL AERONAUTICS AND SPACE ADMINISTRATION 


\section{TABLE OF CONTENTS}

Page

FIGURE INDEX ............................. . . . . iv

LIST OF SYMBOLS ........................... . . . . . . . .

SUMMARY . . . . . . . . . . . . . . . . . . . . . ix

1. INTRODUCTION . . . . . . . . . . . . . . . . . . . . . . . 1

2. THEORETICAL APPROACH . . . . . . . . . . . . . . . . . 3

2.1 General Discussion . . . . . . . . . . . . . . . . 3

2.2 Velocity Calculation . . . . . . . . . . . . . . . 3

2.3 Time-History Calculation . . . . . . . . . . . . . 7

2.4 Time-to-loop . . . . . . . . . . . . . . . . 8

3. SINGLE-PAIR LOOPING . . . . . . . . . . . . . . . . . . . . . . 9

3.1 Significant Parameters . . . . . . . . . . . . . . . . . 9

3.2 Effect of Perturbation Amplitude . . . . . . . . . . . . . 9

3.3 Effect of Vortex Core Radius . . . . . . . . . . . . . . 10

3.4 Effect of Perturbation Wavelength . . . . . . . . . . . 10

4. TWIN-FAIR CONVERGENCE . . . . . . . . . . . . . . . . . . 12

4.1 The Generation and Disruption of Spiral Waves. . . . . . . . 12

4.2 Twin-Pair, Parametric Study . . . . . . . . . . . . . 16

4.3 Three-Dimensional Effects . . . . . . . . . . . . . 17

4.4 Notes on Computing . . . . . . . . . . . . . . . 17

5. MULTIPLE-PAIR CALCULATIONS . . . . . . . . . . . . . . . . 20

5.1 Introduction . . . . . . . . . . . . . . . . 20

5.2 General Approach . . . . . . . . . . . . . . . . . 21

5.3 Three-Dimensional Relaxation of the Near Wake . . . . . . . . 22

5.4 Three-Pair Cases . . . . . . . . . . . . . . . . . . . 23

5.5 "Jumbo-Jet" and "Sawtooth" Cases . . . . . . . . . . . . . 23

5.6 The Sliding-Frame, Aircraft-Fixed Calculation Method . . . 24

6. CONCLUSIONS AND RECOMMENDATIONS . . . . . . . . . . . . . . . 25

7. REFERENCES . . . . . . . . . . . . . . . . . . . 27

APPENDIX: VORTEX LOOPING COMPUTER PROGRAM OPERATION . . . . . . . . 28

FIGURES ........................... . . . 55 


\section{FIGURE INDEX}

Figure No.

Title

Page

2.1 Two-Dimensional Twin Vortex Pair Trajectories

2.2 Definition of the Onset of Looping Instability

58.

3.1 Previous and Present Calculations of the

Distortion of a Vortex Pair

59

3.2 Dependence of Time-to-Loop Upon Initial

Perturbation Amplitude

60

3.3 Effect of Vortex Core Radius on the Distortion of a Vortex Pair

3.4 Dependence of Time-to-Loop Upon Vortex Core Radius

3.5 Dependence of Time-to-Loop Upon Perturbation Wavelength

3.6 End View of Vortex-Pair Distortion Towards Looping, $\lambda=7.8$

3.7 End View of Vortex-Pair Distortion at Sub-looping Wave leng th, $\lambda=5.0$

3.8 Mechanism for Opposing-Sense Vortex and Wave Rotations

3.9 Lower-Limit Wavelengths for Vortex Looping to Occur

3.10 Time-to-Loop as a Function of Perturbation Wavelength and Initial Amplitude

4.1 The Distinction Between Looping and Convergence

4.3 Cross-Sectional Trace for the Convergence Point on Twin Vortex Systems as a Function of $n_{F}$

4.4 Cross-Sectional Trace for the Convergence Point on Twin Vortex Systems as a Function of $\Gamma_{T} / \Gamma_{F}$ 
4.5 Examples of Flap- and Tip-Vortex Convergence

4.6

Twin Pair Parametric Study: Identification of the Convergent Vortex

4.7 Twin Pair Parametric Study: Time-to-Converge, $\lambda=5.375$, and Wavelength/Loading Parameter

4.8

Twin Pair Parametric Study: Time-to-Converge, $C_{L} / A R=0.25$

Twin Pair Parametric Study: Vortex Strengths

Effect of Integration Step Size on Time-toConverge

5.2 Calculation Regions for a Multiple-Pair Vortex Wake

5.5 Three-Dimensional Relaxation to a Steady-State Aircraft Fixed

5.6 Near-Cyclic and Transient Three-Pair Cases

5.7 Effect of Outboard Flap and Landing Gear on the Near Wake of a Boeing 747 on Approach. 


\section{LIST OF SYMBOLS}

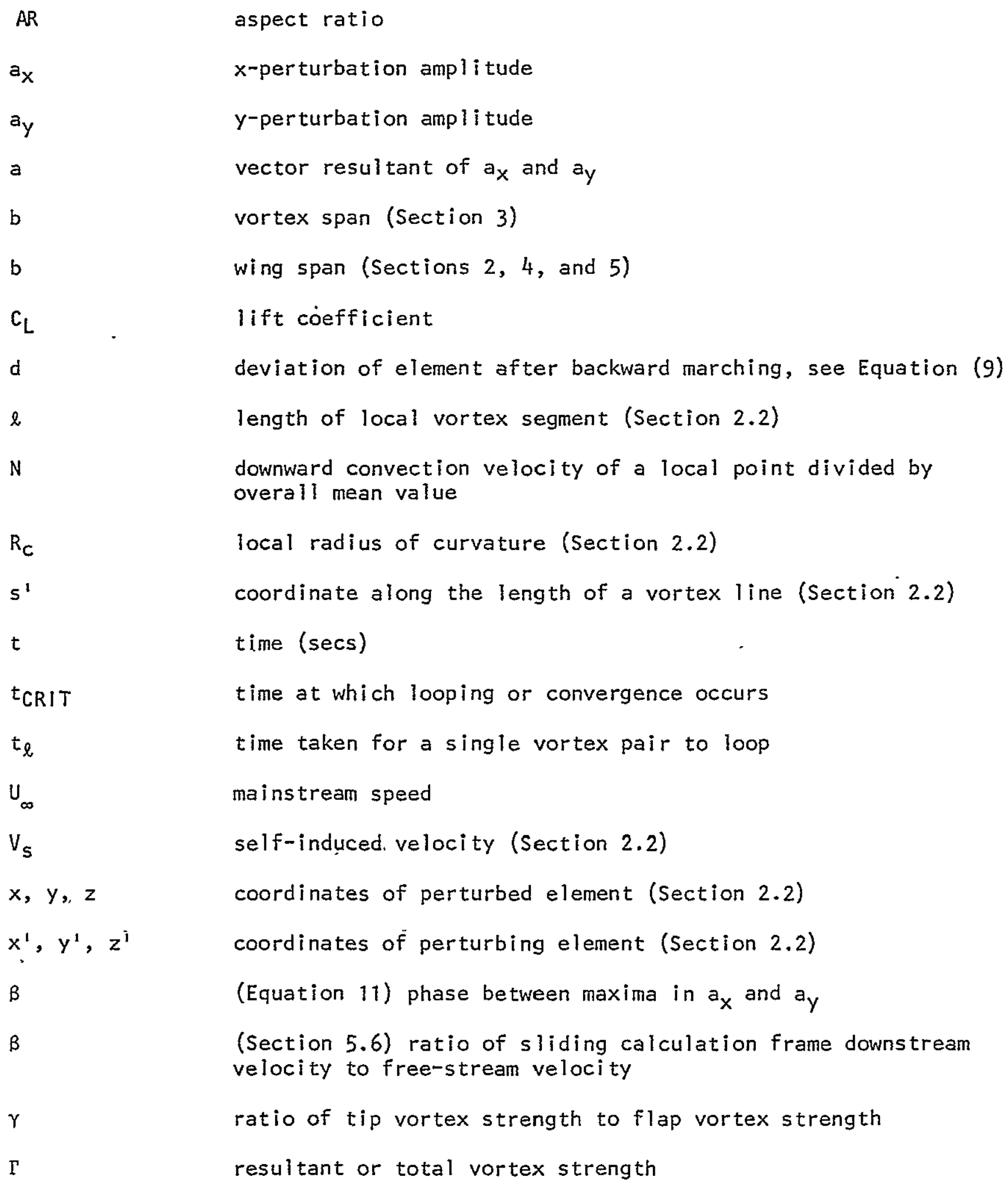

AR

$a_{x}$

${ }^{a} y$

a

b

b

$c_{L}$

d

$\ell$

N

$R_{C}$

$5^{1}$

$t$

${ }^{t}$ CRIT

$t_{\ell}$

$\mathrm{U}_{\infty}$

$V_{S}$

$x, y, z$

$x^{1}, y^{1}, z^{i}$

B

$\beta$

$\gamma$

$\Gamma$

aspect ratio

$x$-perturbation amplitude

$y$-perturbation amplitude

vector resultant of $a_{x}$ and $a_{y}$

vortex span (Section 3)

wing span (Sections 2, 4, and 5)

lift coefficient

deviation of element after backward marching, see Equation (9)

length of local vortex segment (Section 2.2)

downward convection velocity of a local point divided by overall mean value

local radius of curvature (Section 2.2)

coordinate along the length of a vortex line (Section 2.2)

time (secs)

time at which looping or convergence occurs

time taken for a single vortex pair to loop

mainstream speed

self-induced. velocity (Section 2.2)

coordinates of perturbed element (Section 2.2)

coordinates of perturbing element (Section 2.2)

(Equation 11) phase between maxima in $a_{x}$ and $a_{y}$

(Section 5.6) ratio of sliding calculation frame downstream velocity to free-stream velocity

ratio of tip vortex strength to flap vortex strength

resultant or total vortex strength 


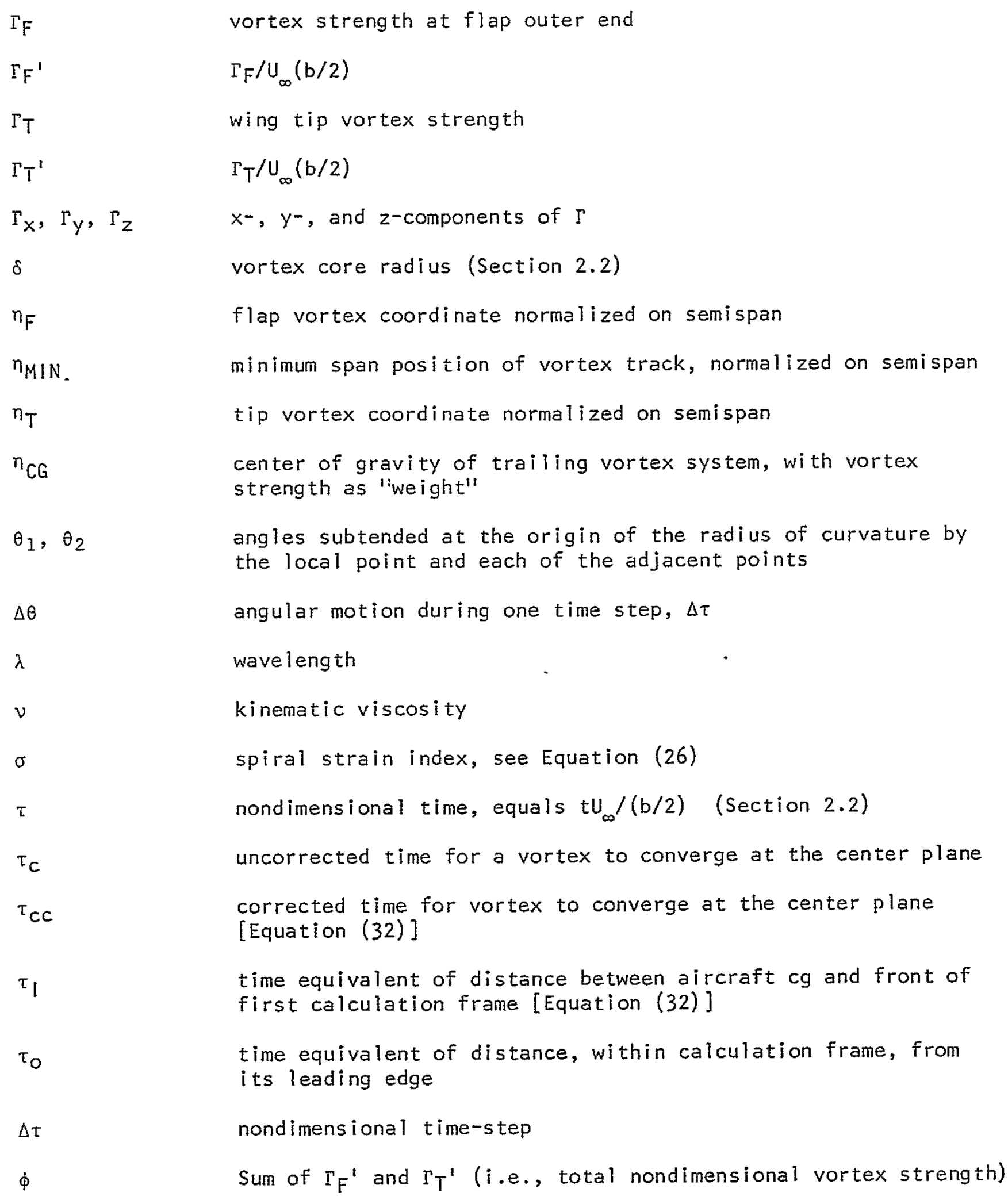




\section{Subscripts}

DUM

$f$

i

$L$

N

$\circ$ intermediate dummy point, see Section 2.2

see Equation (9)

see Equation (9)

denotes influencing point (see Section 2.2)

denotes influenced point (section 2.2)

see Equation (9) 


\title{
NUMERICAL STUDIES OF THREE-DIMENSIONAL BREAKDOWN IN TRAILING VORTEX WAKES
}

\author{
By P. F. Evans and J. E. Hackett \\ Lockheed-Georgia Company
}

\section{SUMMARY}

Finite element, three-dimensional relaxation methods are used to calculate the development of vortex wakes behind ai rcraft for a considerable downstream distance. The inclusion of a self-induction term in the solution, dependent upon local curvature and vortex core radius, permits calculation of finite lifetimes for systems for which infinite life would be predicted twodimensionally. The present report describes the associated computer program together with single-pair, twin-pair, and multiple-pair studies carried out using it. It is found, in single-pair studies, that there is a lower limit to the wavelengths at which the "Crow"-type of instability can occur. Below this limit, self-induction effects cause the plane of the disturbance waves to rotate counter to the vortex direction. Self induction in two dimensionally generated twin spiral waves causes an increase in axial length which becomes more marked with decreasing initial wavelength. The time taken for vortex convergence toward the center plane is correspondingly increased. The limited parametric twin-pair study performed suggests that time-toconverge increases with increasing flap span. Limited studies of Boeing 747 configurations show correct qualitative response to removal of the outer flap and to gear deployment, as compared with wind tunnel and flight test experience. 


\section{INTRODUCTION}

During the past several years, strenuous efforts have been made to devise means for breaking up prematurely and permanently the trailing vortices behind large transport aircraft so as to reduce or eliminate their hazard to following aircraft. Many of the attempts aimed at modifying individual vortices have met with indifferent success or have been impractical or too costly to apply.

One of the more reliable "natural" modes of vortex pair decay is vortex looping. This form of instability was analyzed first by crow (ref. 1), using linear methods. Shortly thereafter, his results were extended numerically by Hackett and Theisen (ref. 2), who calculated the progression from Crow's sinuous shapes toward the familiar "looped" form sometimes observed in flight (ref. 3) or in water tank experiments (refs. 2 and 4). The calculations used finite-element, time-dependent techniques applied to single vortex pairs. A limited study was made of the effect of the initial amplitude and wavelength of a "Crow"-type perturbation. Because these initial quantities must be specified, there are difficulties in specifying time-to-loop.

It is the objective of the present work to provide methods for deepening the previous single-pair studies and extending the methods to multiple-pair configurations including both wakes typical of current jumbo jets and experimental cases such as "sawtooth" loading suggested by Rossow (ref. 5) for promoting early wake disruption. Appropriate Fortran algorithms have been written and documented which achieve the above objectives. The program was delivered to NASA-Ames in April 1976 and implemented there on both the IBM 360-67 and CDC-7600 computers. Program development was carried out at the Lockheed-Georgia Company on a MAC-16 mini-computer, where CRT graphics are available and on a Univac 1106 terminal system for longer runs. The program and its documentation form Appendix $A$ of the present report.

in any study like the present one, it is very desirable to make comparisons with experimental results. Though documented flow investigations in the near-field are quite numerous and despite the wealth of longer-time measurements at discrete downstream planes, it has proved impossible to find quantitative experiments which give adequate details of vortex looping flow.

Experiments with towed models are the most suitable for this and al though looping has been noted with a small model size relative to the test region cross section (ref. 2), the majority of scale models tested have had larger relative size and looping has not occurred. In some cases, wall constraints may have inhibited looping; in others the vortices have encountered a horizontal surface before looping had time to occur. Even with the looping flow properly generated, good measurements are difficult.

In carrying out numerical calculations of the vortex wake, a choice is available between a space-fixed and an aircraft-fixed reference system. During the present work, it became apparent that while vortex looping is an unsteady space-fixed event, spiral multiple-vortex development is steady in an aircraftfixed frame of reference. Though computing considerations made space-fixed analysis mandatory for a very long wake, it was found in practice that an 
aircraft-fixed analysis was essential to provide proper initial conditions for multiple vortex arrays. An extended version of the program was therefore developed for this purpose, which is compatible with the main, space-fixed algorithm.

Sufficient experience has now been obtained with the program that the proper initialization procedures, step sizes, etc. are in hand and the significance of each input variable is well appreciated. These factors are documented herein. However, since some of the quoted twin and multiple-pair examples lie part-way up the learning curve, the corresponding results should not be regarded as definitive but rather as a qualitative guide for future application of the program.

Section 2 will outline the theoretical approach, velocity calculation methods, self-induction effects, stepping procedures, etc. used throughout the present work. Section 3 is devoted to single vortex pair events and further examines the vortex looping phenomenon. In Section 4 the distinction between looping and spiral center-plane convergence is discussed and the effects of twin pair variables are reviewed. The computational details and requirements for such calculations are also included.

In Section 5 pilot calculations are described involving multiple vortex pair wakes for actual aircraft configurations. Conclusions are given in Section 6. 


\section{THEORETICAL APPROACH}

\subsection{General Discussion}

When dealing with single-pair decay, there is little option but to impose an initial perturbation either arbitrarily or via a knowledge of a specific turbulence environment for aircraft flight. For multiple pairs, however, this situation is relieved by the fact that in some cases vortex helices form naturally, starting in the near wake. In other cases, vortex strengths and positions may be such that the pattern disperses rapidily without helices forming. Figure 2.1 shows an example of each type, in end view, for twin vortex pairs. Bilanin et al (ref. 6) have produced classification charts showing the relative vortex strengths and relative spans associated with each type of motion.

In analyzing single-pair deformations, a space-fixed frame of reference is perfectly adequate. However, it is evident from the above that an aircraftfixed analysis is needed for multiple pairs, at least to start the calculation. Accordingly, a variant of the original program has been written, in an aircraft-fixed frame of reference, which includes the near-field effect of the wing. The resulting program solves the steady-state problem in three dimensions by a time relaxation of an initial two-dimensional, Trefftz plane solution.

Once the initial, transient motions are complete there may remain a number of vortex pairs arranged in helices. It is not practical to continue to analyze the subsequent motion in aircraft-fixed axes, because of the prohibitive number of points involved, which must ail be perturbed starting from zero time. A change is-therefore made into space-fixed axes and a regular cyclic geometry is assumed. If this change is made at a distance corresponding to $N$ miles aft of the aircraft and the subsequent time-to-looping is $T$ seconds, the total distance to looping is given by $\left(N+U_{\infty} T\right)$ miles.

It must be remarked that the above transient-plus-cyclic distinction is arbitrary, since divergence may require many cycles in some cases. However, the distinction is useful and practical except possibly for borderline, nearcyclic cases.

\subsection{Velocity Calculations}

Given a constant-strength vortex, strength $I$, radius $\delta$, which follows an arbitrary curved path in three dimensions from point $A\left(x_{A}, y_{A}, z_{A}\right)$ to point $B\left(x_{B}, y_{B}, z_{B}\right)$, we seek the three velocity components, $u, v$, and $w$, at an arbitrary point $N(x, y, z)$ on the vortex. $L\left(x^{\prime}, y^{\prime}, z^{\prime}\right)$ is a typical element which influences $N$. 


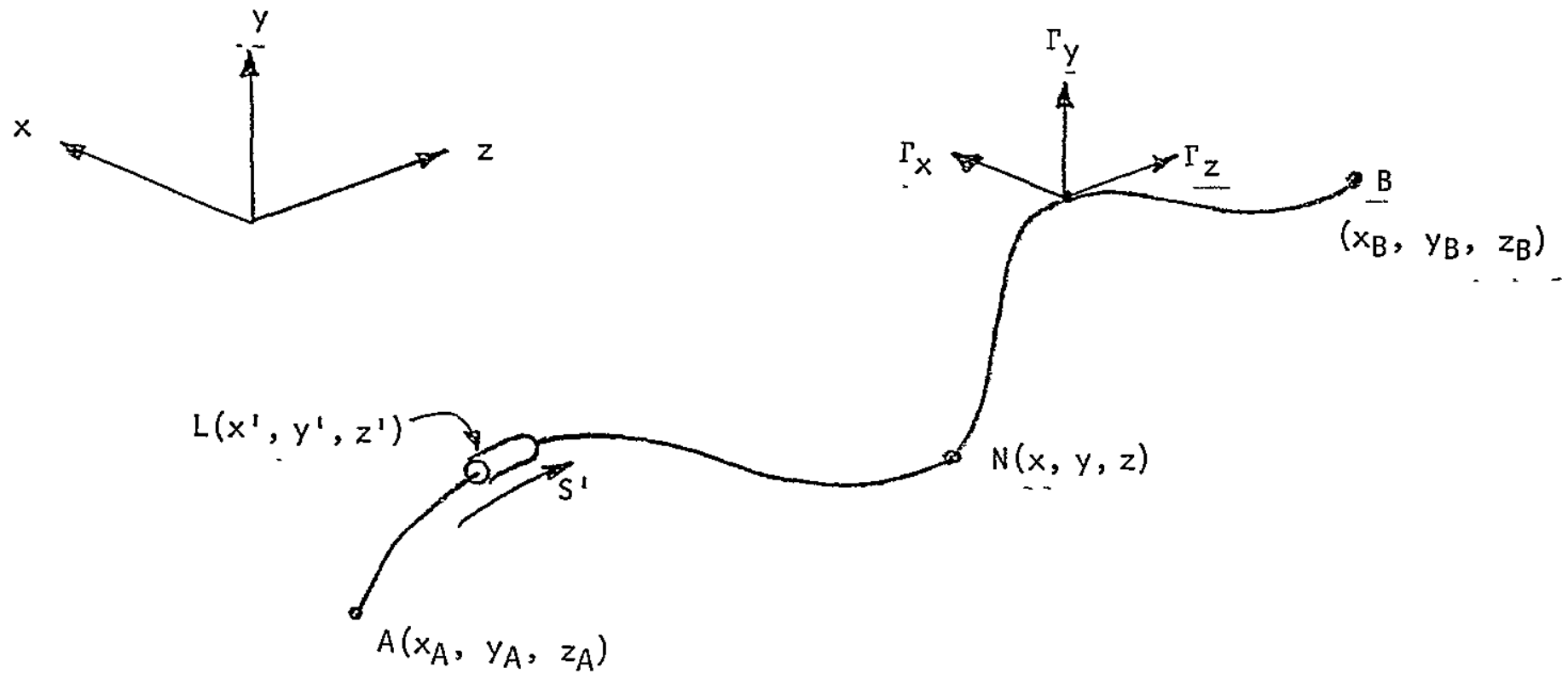

The calculation is divided into three parts: the near, intermediate, and far fields. The near field concerns the effect of an element on itself, while the far field concerns elements sufficiently remote that their detailed structure is not significant at point $N$. All other points are classified as intermediate.

When studying the periodic vortex cases, it is convenient to use imaging techniques such that only one side of the aircraft wake and only one-half wave (one full wave for spiral perturbations) are actually perturbed and the periodic part of the wake vortex system is represented by various reflections. In the case of aircraft-fixed analysis, there is no periodic geometry, but half-plane symmetry still applies.

Near-field: - The self-induced velocity is determined in a manner similar to that of Leonard (ref. 8). The self-induced velocity $\left(V_{s}\right)$ is in a direction normal to the local plane of curvature and is given by the relation

$$
V_{S}=\frac{\Gamma}{4 \pi R_{c}}\left[\log _{e}\left\{\frac{8 R_{c}}{\delta}\right\}-0.558-\frac{1}{2} \log _{e}\left\{\cot \frac{\theta_{1}}{4} \cot \frac{\theta_{2}}{4}\right\}\right]
$$

where:

$$
\begin{aligned}
& R_{c}=\text { local radius of curvature } \\
& \theta_{1}, \theta_{2}=\text { angles between the local point and each of the adjacent } \\
& \text { points based on the origin of the radius of curvature } \\
& \delta=\text { core radius, derived from } \\
& \frac{d \delta^{2}}{d t}=4 \nu-\frac{\delta^{2}}{l} \frac{d l}{d t} \\
& \ell=\text { length of local segment }
\end{aligned}
$$


The terms in the time-dependent expression for core radius, $\delta$, yield respectively the viscous growth and vortex stretching effects. Since molecular viscosity has been used in the present calculations, viscous growth is very small. Equation (la) is evaluated in finite-difference form.

Intermediate field. - We resolve the total strength $\mathrm{P}$ into orthogonal components $\Gamma_{z}, \Gamma_{z}$, and $\Gamma_{z}$ at $x^{\prime}, y^{\prime}, z^{\prime}$ so that

$$
\Gamma_{X}^{2}+\Gamma_{y}^{2}+\Gamma_{z}^{2}=\Gamma^{2}
$$

and

$$
\Gamma=\frac{\Gamma_{x}}{d x^{\prime} / d s^{\prime}}=\frac{\Gamma_{y}}{d y^{\prime} / d s^{\prime}}=\frac{\Gamma_{z}}{d z^{\prime} / d s^{\prime}}
$$

where

$$
d x^{\prime 2}+d y^{\prime 2}+d z^{\prime 2}=d s^{2}
$$

Reference 7 gives:

$$
\begin{aligned}
u & =\frac{\Gamma}{4 \pi} \int_{A}^{B}\left\{\frac{d y^{\prime}}{d s^{\prime}} \frac{z-z^{\prime}}{r}-\frac{d z^{\prime}}{d s^{\prime}} \frac{y-y^{\prime}}{r}\right\} \frac{d s^{3}}{r^{2}} \\
v & =\frac{\Gamma}{4 \pi} \int_{A}^{B}\left\{\frac{d z^{\prime}}{d s^{\prime}} \frac{x-x^{\prime}}{r}-\frac{d x^{\prime}}{d s^{3}} \frac{z-z^{3}}{r}\right\} \frac{d s^{\prime}}{r^{2}} \\
w & =\frac{\Gamma}{4 \pi} \int_{A}^{B}\left\{\frac{d x^{\prime}}{d s^{\prime}} \frac{y-y^{\prime}}{r}-\frac{d y^{\prime}}{d s^{\prime}} \frac{x-x^{3}}{r}\right\} \frac{d s^{\prime}}{r^{2}}
\end{aligned}
$$

where $A B$ is the vortex $l$ ine and $r^{2}=\left(x-x^{1}\right)^{2}+\left(y-y^{1}\right)^{2}+\left(z-z^{1}\right)^{2}$.

Taking the u component as typical

$$
u=\int_{A}^{B} \frac{d y^{\prime}}{d s^{\prime}} \frac{\Gamma}{4 \pi r^{3}}\left(z-z^{1}\right) d s^{\prime}-\int_{A}^{B} \frac{d z^{\prime}}{d s^{\prime}} \frac{\Gamma}{4 \pi r^{3}}\left(y-y^{\prime}\right) d s^{\prime}
$$

Now

$$
\Gamma_{y}=\frac{d y^{\prime}}{d s^{\prime}} \Gamma \text { and } \Gamma_{z}=\frac{d z^{\prime}}{d s^{\prime}} \Gamma
$$


So

$$
u=\int_{A}^{B} \frac{\Gamma y}{4 \pi r^{3}}\left(z-z^{\prime}\right) d s^{\prime}-\int_{A}^{B} \frac{\Gamma_{z}}{4 \pi r^{3}}\left(y-y^{\prime}\right) d s^{\prime}
$$

Writing Equation (5), at a point $N$, as a sum over LTOTAL finite elements:

$$
\left.\begin{array}{l}
u_{N}=\sum_{L=1}^{L T O T A L} \frac{(\Gamma \Delta y)}{4 \pi r_{N L}^{3}}\left(z_{N}-z_{L}\right)-\sum_{L=1}^{L T O T A L} \frac{(\Gamma \Delta z)}{4 \pi r N L^{3}}\left(y_{N}-y_{L}\right) \\
v_{N}=\sum_{L=1}^{L T T A L} \frac{(\Gamma \Delta z)}{4 \pi r_{N L}^{3}}\left(x_{N}-x_{L}\right)-\sum_{L=1}^{L T O T A L} \frac{(\Gamma \Delta x)}{4 \pi r_{N L}^{3}}\left(z_{N}-z_{L}\right) \\
w_{N}=\sum_{L=1}^{L} \frac{(\Gamma \Delta x)}{4 \pi r_{N L}^{3}}\left(y_{N}-y_{L}\right)-\sum_{L=1}^{L} \frac{L}{L O T A L} \frac{(\Gamma \Delta y)}{4 \pi r_{N L}^{3}}\left(x_{N}-x_{L}\right)
\end{array}\right\}
$$

where $\Delta x, \Delta y$, and $\Delta z$ are the components of a straight, finite element which represents a segment of the vortex $A B$. When $N=L$, Equation (1) is used. In implementing Equation (6) for $N \frac{1}{\tau} \mathrm{L}$, a "short element" concept is applied, in which the products, $\Gamma \Delta x_{L}, \Gamma \Delta y_{L}$, and $\Gamma \Delta z_{L}$ are defined as the short element strength components. Dummy points (XDUM, YDUM, ZDUM) are situated midway between the perturbed points along each vortex line. Therefore:

$$
\begin{aligned}
& \Delta x_{L}=X D U M_{L+1}-X D U M_{L} \\
& \Delta x_{L}=Y D U M_{L+1}-\text { LDUM }_{L} \\
& \Delta z_{L}=Z_{L U M_{L+1}}-\text { ZDUM }_{L}
\end{aligned}
$$

By using this short element method the effects of vortex stretching in the intermediate field can be considered, without changing the total number of points, by suitable modifications to $\Gamma \Delta x_{L}, \Gamma \Delta y_{L}$, and $\Gamma \Delta z_{L}$.

Far field. - Beyond one or two complete wavelengths, each side of a perturbed point the vortex system may be extended by horizontal, straight-line elements. These are added at both ends of periodic geometries (space fixed) and at the downstream end of aircraft-fixed geometries. 


\subsection{Time-History Calculation}

A one-step, second-order predictor-corrector computation is used in which the desired element $x$-displacement, for example, is defined as the midpoint of the resultant of two Euler displacement vectors, $u(t) d t$ and $u(t+d t) d t$. $u(t)$ is the induced velocity field based on the positions of the vortex element system at the time $t$ and $u(t+d t)$ is the induced velocity based on the positions of the system at time $(t+d t)$. The general relation for displacement using predictor-corrector stepping is:

$$
x^{\prime}(t+d t)=\frac{1}{2}[x(t)+x(t+d t)+u(t+d t) \cdot d t]
$$

where

$$
\begin{aligned}
x(t+d t) & =x(t)+u(t) \cdot d t \text { (Euler value at }(t+d t)) \\
x(t) & =\text { value at } t \\
x^{3}(t+d t) & =\text { predictor-corrector value at } t+d t
\end{aligned}
$$

The other two components are treated similarly.

In two-dimensional calculations the accuracy of the time-integration may be checked fairly readily by determining the vortex center of gravity, moments, and the Kirkoff-Routh path function (see ref: 5). Such checks are generally not available for three-dimensional motions. In principle, checks should be made that conservation laws are obeyed, but the necessary volume integrations are more difficult and more numerically hazardous than the original calculation. The only check found feasible so far is to employ a reversed-marching technique to find the degree of accuracy to which the system returns to its original state. At the end of a series of time steps, the sign of the time increment is reversed and the program marches back to the initial time. The deviation for each vortex element is then defined as:

$$
d_{i}=\left[\left(x_{i_{f}}-x_{i_{0}}\right)^{2}+\left(y_{i_{f}}-y_{i_{0}}\right)^{2}+\left(z_{i_{f}}-z_{i_{0}}\right)^{2}\right]^{\frac{1}{2}}
$$

where $f$ denotes position after backward marching, and o denotes initial position. The arithmetic

$$
\left(=\frac{1}{N} \sum_{i=1}^{N} d_{i}\right)
$$

and geometric

$$
\left(=\left(d_{1} d_{2} \cdots d_{N}\right)^{1 / N}\right)
$$

means are then determined. Some typical results are illustrated in the following, table: 
TABLE 1. - ACCURACY OF TIME-STEPPING METHODS (SINGLE PAIR, 51 STEPS)

\begin{tabular}{|c|c|c|}
\hline Calculation Method & Arith. Mean & Geom. Mean \\
\hline Predictor-Corrector & .00319 & .00271 \\
\hline Euler & .801 & .696 \\
\hline
\end{tabular}

The predictor-correlator method, which takes little more than twice the running time of the simple Euler stepping method, provides a 250-fold accuracy gain in this particular example. In practice, a part of such benefits is used to reduce computer run time.

\subsection{Time-to-Loop}

The formation of long loops along the track of an originally parallel trailing vortex pair is a well-known occurrence. The development from the initial sinuous perturbation (ref. 1) towards the near-ring shapes may be calculated (ref. 2) by finite element methods. The next stage, the formation of loops, is not well understood. In real flows, the point of nearest approach, across the plane of symmetry, starts to convect downwards; but almost immediately there is a "click" and the vortices break, change partners, and then link across the center-plane to form loops. This event is a very fundamental one since it also typifies the cascading process by which large eddies break down to smaller ones in turbulent flows. Nevertheless, there is little detailed knowledge of the mechanism, and no criterion exists which defines when the "click" occurs.

Fortunately, it is sufficiently accurate for present purposes to define the start of looping as the time at which the point of closest approach is convected downward at ten times the mean downdrift velocity for the whole wake. This defines the start of looping sufficiently sharply. for use in long-time solutions. (See Figure 2.2.) 


\section{SINGLE-PAIR LOOPING}

\subsection{Significant Parameters}

Reference 2 gives examples which extend numerically the linearized analysis by Crow (ref. 1). As indicated above, these space-fixed calculations have been improved, both numerically and by the addition of the element self-induction term of Equation (1). As a result, the tendency to a fine-grain waviness instability on each individual loop has been reduced very considerably. The use of the higher precision. CDC-7600 computer for many of the runs reported here gave additional numerical improvements and permitted longer time histories to be calculated. Figures 3.1 (a), (b), and (c) show, respectively, plan views of the initial condition, a later geometry using results from a reference 2 type of calculation and the corresponding result from the present program.

For a given vortex spacing (b), the time to loop $\left(t_{\ell}\right)$, as defined in Section 2.4 , is a function of the vortex strength $(\Gamma)$; the amplitudes $\left(a_{x}, a_{y}\right)$, phase $(\beta)$, and wavelength $(\lambda b)$ of the initial perturbation and the vortex core radius $(\delta)$. The relationships take the nondimensional form:

$$
\frac{\Gamma t_{\ell}}{b^{2}}=f\left\{\lambda, \frac{a x}{b}, \frac{a y}{b}, \beta, \frac{\delta}{b}\right\}
$$

\subsection{Effect of Perturbation Amplitude}

In Section 3, the initial perturbations $a_{x}$ and $a_{y}$ usually will be equal and in phase, giving the classical $45^{\circ}$-inclined plane wave shown in Figure 3.1(a). In Figure 3.2, it is evident that there is an approximately inverse relationship between time-to-loop and initial amplitude. The constant of proportionality is, of course, dependent upon vortex core radius and wavelength. As perturbation amplitude increases, the drift velocity of the point-of-closeapproach will increase. Eventually, this will reach ten times the mean (straight vortex) drift velocity as used to define the onset of looping. This produces the intercept with the horizontal axis. In potential flow, this would correspond approximately to the condition when the distance of closest approach was $10 \%$ of the mean vortex spacing.

Limited checks have also been made of the effect of using spiral and planar initial conditions. Labelled points in Figure 3.2 show that the use of a spiral initial condition increases the time-to-loop by nearly $40 \%$. This is important because multiple vortex pairs develop spiral modes. Clearly, the "Crow" mode is more efficient at driving the system towards looping. It is interesting to note that, if the $x$ - and $y$-perturbations are applied separately, the $x$-perturbation on $i$ ts own is very inefficient in this regard. However, applying solely y-perturbation increases time-to-loop by only $20 \%$ over the combined perturbation. 
These results suggest that the present methods could be used to determine optimum configurations for deliberately induced initial perturbations in real flows.

\subsection{Effect of Vortex Core Radius}

Figure 3.3 shows the results of three calculations made for the same total time. The first uses current numerics, but omits the self-induction effect on each element. Figures $3.3(b)$ and $(c)$ show corresponding results for medium and large-sized cores. As might be expected, increase in core size has a damping effect on the overall motion. It is also interesting to note that the omission of curvature effects produces a result intermediate to the other two; i.e. an effective core radius has been implied. This is a somewhat similar situation to the use of a cut-off radius by Crow (ref. 1). In the present case, the effective radius is determined (if self-terms are neglected) by the number of elements per wave.

Figure 3.4 returns to the full equations and shows a gradual increase in time-to-loop with vortex core radius. It should be noted that the right hand part of this curve becomes less valid because the small radius assumptions inherent in Equation (1) are violated.

A number of investigators have suggested means for diffusing individual vortices to reduce their hazard to following aircraft. The present result suggests that such diffusion may actually increase the time to ultimate decay, by delaying looping. It is therefore important to be certain that such attempts to accelerate diffusion have a sufficient effect.

\subsection{Effect of Perturbation Wavelength}

Though time-to-loop decreased with decrease in wavelength, as previously (ref. 2), an unexpected upturn in the curve occurred at short wavelengths in the present calculations (see Figure 3.5)..

To better understand the mechanisms, let us first examine in more detail the progression towards "conventional" looping. Figure 3.6 shows, at zero time, an end view of the right-hand vortex, inclined outward at 45 degrees. The individual points are calculation points on a half-wave. As time progresses the whole array drifts downward, largely maintaining its 45 -degree inclination. At the two longest times shown, the lower points are moving downward and inward at an increasing rate: a nascent looping stage has been reached.

Figure 3.7 has essentially the same conditions as previously, but a shorter wavelength. Initially, the innermost vortices move inward as before but at a faster rate, as would be expected from previous experience concerning wavelength effects. However, the 45-degree inclination is not retained as before. A clockwise rotation, opposing the direction of the vortex, develops. 
In consequence, the distance-of-closest approach starts to increase markedly and looping is forestalled.

The suggested mechanism for the above motion is illustrated in Figure 3.8(a). At any wavelength, there is a tendency for the crests of each sine wave to self-convect like part of a vortex ring. The corresponding motion is outward and downward at the top of the wave, inward and upward at the bottom, and zero at the inflection points. This motion increases as the wavelength is reduced. Counterbalancing this effect is the velocity gradient due to the opposite vortex, which is approximately constant. Thus, the outward rotation due to self-induction, which is dependent upon the ratio of amplitude to wavelength, is offset against the velocity gradient effect, which depends only upon amplitude. An illustrative velocity vector diagram is shown in Figure $3.8(b)$.

Boundaries have been found, in an amplitude wavelength domain, which define the lower $1 \mathrm{imit}$ for looping. Figure 3.9 shows the results of a number of numerical calculations, for two core radii. As might be expected from coredring convection properties, the larger vortex core radi cases are less likely to self-rotate.

To each point on the boundaries shown in Figure 3.9, there corresponds a (different) time-to-loop. These are minima, as was noted in Figure 3.5 . Figure 3.10 illustrates the nature of time-to-loop contours in the same coordinates as the previous figure. 


\section{TWIN PAIR CONVERGENCE}

\subsection{The Generation and Disruption of Spiral Waves}

It was noted previously that single spiral waves were noticeably more stable than plane waves of the "Crow" type. In this section we shall examine the properties of spiral waves with emphasis on the mechanisms and flow geometries which lead to vortex destruction. Though the same calculation procedures and divergence criteria may be used for each, there is a very important distinction between the instability of a spiral wave and that of a plane wave of the "Crow"type. Figure 4.1 illustrates this distinction from the viewpoint of an observer fixed in space. When a vortex trail with plane waves reaches a sufficient age, this observer sees "necking" start to occur, followed by the aforementioned "click" when looping occurs. Thereafter, the looping point remains fixed in space. In contrast, the spiral wave is fed continuously towards the center plane. Prior to the critical time, the fixed-observer sees a convergence event moving towards him in the direction of aircraft motion. The "time-to-converge" corresponds to its passage directly in front of him. In contrast to the previous case, the event is steady as seen from an aircraft frame of reference. These considerations in no way preclude space-fixed events due to atmospheric disturbance or other modes of instability. However, to underline the distinction, the distruction of spiral waves by meeting at the center plane will be referred to as "convergence" and "looping" will be reserved for the space-fixed event.

Definition of variables. The spiral wavelength for a twin-pair trail is determined by aircraft forward speed, as well as vortex details and it must be included in the normalization scheme. Thus, if primed quantities are dimensionless and the kinematic variables are as shown in Figure 4.2, we define

$$
\Gamma_{F}^{\prime}=\frac{\Gamma_{F}}{U_{\infty} b / 2} \quad \Gamma_{T}^{\prime}=\frac{\Gamma_{T}}{U_{\infty} b / 2}
$$

with a total

$$
\phi=\Gamma_{F^{\prime}}+\Gamma_{T}{ }^{\prime}
$$

For convenience, we write.

$$
\gamma=\frac{\Gamma_{T}}{\Gamma_{F}}=\frac{\Gamma_{T}^{\prime}}{\Gamma_{F}}
$$

The following relationships are noted:

$$
\begin{aligned}
& I_{F}^{\prime}=\frac{\phi}{1+\gamma} \quad I_{T^{\prime}}=\frac{\gamma \phi}{1+\gamma} \\
& n_{F}+\gamma \eta_{T}=(1+\gamma) \eta_{C G} . \\
& C_{L} / A R=\phi n_{C G}
\end{aligned}
$$


We normalize time $t$ (secs), after aircraft passage, via

$$
\tau=\frac{t U_{\infty}}{b / 2}
$$

For multiple vortex arrays and because of the possibility of c.g. variations under viscous, three-dimensional conditions, we measure wavelength of spiral motions, etc., in aircraft spans, as $\lambda b$. It may be shown that the time per revolution of $\Gamma_{F}$ and $\Gamma_{T}$ about their c.g., in two dimensions, is given by

$$
\tau / \text { rev. }=-4 \pi^{2} \frac{(1+\gamma)^{2}}{\phi}\left(n_{T}-n_{C G}\right)^{2}
$$

or in aircraft variables

$$
\tau / \text { rev. }=4 \pi^{2} \frac{A R}{C_{L}} \frac{\left(n_{F}+\gamma n_{T}\right)}{(1+\gamma)}\left(n_{T}-n_{F}\right)^{2}
$$

and we note also that the wavelength,

$$
\lambda=\frac{1}{2} \tau / \text { rev. }
$$

Conditions for convergence. Figures 4.3 and 4.4 show the effects, on the calculated development towards convergence, of varying the relative span positions and strengths of the vortices. The vortex wavelength, after threedimensional relaxation, is a function of these variables and both end view and time-to-loop follow from this. Vortex radius also becomes a significant variable.

With a wing tip vortex somewhat weaker than the flap vortex, Figure 4.4 indicates that small flap spans lead to early convergence of the tip vortex. As the flap span increases, the spiral amplitude is reduced, and the trajectories approach the center plane less closely. Consequently, convergence is delayed. A simple geometric analysis shows that when the flap vortex is the stronger, the tip vortex will intersect the center plane after 180-degrees or less of travel provided that

$$
n_{F} \leq \frac{1-\gamma}{2}, \quad(\gamma \leq 1) \text {. }
$$

Equation (21) is a particular case of a geometric expression for minimum centerplane clearance by the tip vortex, i.e.

$$
n_{M I N}=\frac{2}{1+\gamma}\left(n_{F}+\gamma\right)-1, \quad(\gamma \leq 1)
$$

Figure 4.4 illustrates the consequences of Equation (22). With flap-span constant, a case with $\gamma$ less than one [Figure 4.4(a)] clearly converges much more quickly than with equal strength [Figure 4.4(b)]. We note here that since the two spirals are of equal amplitude, they each have an equal chance of converging. 
Once $\gamma>1$, the flap vortex (now weaker) forms the outermost spiral and consequently $\eta_{M I N}$ clearly cannot be less than $n_{F}$. This explains the range of validity of Equation (22).

The velocity condition for convergence. Equations (21) and (22), being based solely upon geometric considerations, do not reflect the velocity condition used to define convergence in the present calculations. The effect is to move the critical point outward from the center plane by an amount

$$
\Delta \eta=\frac{\gamma\left(\eta_{F}+\gamma\right)}{N(\gamma+1)^{2}}
$$

which is added to Equation (22) to give

$$
n_{M I N}=\frac{1}{1+\gamma}\left(n_{F}+\gamma\right)\left\{2+\frac{\gamma}{N(\gamma+1)}\right\}-1 \quad \gamma \leq 1
$$

Since $\gamma \leq 1$ and an $N$-value of 10.0 is used, the effect of the added term is evidently smal1. A corresponding analysis modifies Equation (21) to the form

$$
\eta_{F}-\frac{(\gamma-1)^{2} N}{(2(\gamma+1) N-\gamma)}-\gamma \quad(\gamma \leq 1)
$$

This reduces to Equation (21) for $N \rightarrow \infty$ or $\gamma \rightarrow 0$. Again, the differences are small within the range of validity. The differences between results derived from (24) and (25) and those from the simpler expressions are not sufficient to justify the added complexity in most applications.

The spiral strain index, $\sigma$. Equations (21) and (25) describe the conditions under which the flap vortex drives the tip vortex into, or near to the the center plane after half a revolution or less of travel. The tip vortex, being the weaker, forms the outermost spiral, which converges. However, it is evident from Figures 4.3 and 4.4 that, for cases with several revolutions prior to convergence, it is also possible for the inner, more intense, spiral to converge first. Figure 4.5 shows a further example of each type.

Figure 4.6 is a parametric map with flap span as ordinate and relative vortex strength as abscissa. Each plotted point depicts one computer run in a parametric study which will be described in Section 4.2. Triangles and circles represent, respectively, flap and tip vortex convergence as the first event. As might be expected, tip vortex convergence occurs in the vicinity of the lower-left boundary [Eqns. (21) and (25)], but then flap vortex convergence becomes more likely as $\eta_{F}$ is increased. There is also a change on passing through $\gamma=1.0$ (i.e. flap and tip strengths equal). To understand the structure of these domains, we shall examine the distortion mechanism which leads ultimately to convergence.

On passing through its innermost position, each vortex receives an extra. downward impulse induced by the opposite-hand vortex at a distance $2 n_{M I N}$ away. This causes an outward-spiraling tendency which, continued long enough, leads to convergence at the center plane. We may quantify this tendency in terms 
of the nondimensional velocity increment causing the effect. Thus, we define spiral-strain indices:

$$
\text { and } \begin{aligned}
\sigma_{F} & =\frac{\Gamma_{F}^{\prime}}{n_{M I N F}} \\
\text { and } & \sigma_{T}=\frac{\Gamma_{T}^{\prime}}{n_{M I N T}}
\end{aligned}
$$

We now hypothesize that the vortex which has the larger $\sigma$ value will converge first. Thus, for the tip vortex to converge first requires that

$$
\sigma_{T}>\sigma_{F}
$$

Using Equation (22) and noting that $\eta_{M I N F}=\eta_{F}$, this yields

$$
\frac{\Gamma_{F^{\prime} \gamma}(1+\gamma)}{2 \eta_{F_{C R I T}}-(1-\gamma)}>\frac{\Gamma_{F}^{\prime}}{\eta_{F_{C R ! T}}}
$$

for the tip vortex to converge first.

A borderline case may be found by using an equality in Equation (27) which readily yields

$$
\eta_{F_{C R I T}}=\frac{1}{\gamma+2}
$$

This line partitions the data in Figure 4-5 quite successfully though not perfectly because of the simplifying assumptions. However, resolution of the flap- or tip-convergent regions requires some care. written

Provided that $n_{\text {CRIT }}$ and $\Gamma_{F}$ are both positive, Equation (27) may be

$$
\frac{N}{D}=\frac{\eta_{F_{C R I T}} \gamma(1+\gamma)}{2 \eta_{F_{C R I T}}-(1-\gamma)}>1
$$

We may clear this further, by multiplying by $D$, only if

$$
2 n_{\text {FRIT }}-(1-\gamma)>1
$$

i.e. if $\gamma>1-2 n_{\text {CRIT }}$. Thus,

This defines a region in Figure 4.5 above the line marked "Eqn. (21)." 


$$
n_{F_{C R I T}} \gamma(1+\gamma)>\left(2 n_{F_{C R I T}}-(1-\gamma)\right), \quad \gamma>\left(1-2 n_{F_{C R I T}}\right)
$$

giving

$$
n_{F_{C R I T}}\left(\gamma^{2}+\gamma-2\right)>(\gamma-1), \quad \gamma>\left(1-2{ }^{n} F_{C R I T}\right)
$$

Since both sides change sign at $\gamma=1$, the cases $\gamma \$ 1$ must be considered separately. This yields, eventually

$$
n_{\text {FRIT }}>\frac{1}{\gamma+2}, \quad \gamma>1
$$

and

$$
\text { - } \quad n_{F_{C R I T}}<\frac{1}{\gamma+2}, \quad\left(1-2 n_{F_{C R I T}}\right)<\gamma<1
$$

for the tip vortex to converge first. This agrees with most of the test cases in Figure 4.6. More elaborate treatments are obviously possible, using Equation (25), for example, or taking spiral diameter into account. However, the simplicity of Equation (30) is attractive.

\subsection{Twin-Pair, Parametric Study}

By suitable choices of flap span and loading, it is possible to reduce the vortex hazard for any specific aircraft at a given weight and speed. Recent studies (ref. 11) have demonstrated the hazard reduction benefits of reduced flap span, for example. In order to provide a basis for quantifying the trades between flap span and setting angle, convergence times have been calculated over a wide range of $n_{F}$ and $\Gamma_{T} / \Gamma_{F}$. Boundaries for rapid convergence have been published previously (ref. 6), but no attempt has been made to calculate the time taken for cases within such limits. This is only possible if three-dimensional, self-induction effects are included.

Figure 4.7a shows contours of the dimensionless time-to-converge [see Equation (17)]. For computational consistency a constant wave-length $\lambda=5.375$, was employed for the whole figure, the implicit assumption being that $\lambda$ is a weak parameter. However, subsequent studies (see Section 4.3) have shown this to be untrue. Consequently, the loading parameter $\left(C_{L} / A R\right)$ cannot be merged with nondimensional time $\tau$, as was originally hoped. It follows that a map like Figure 4.7 a should be prepared for each of a series of constant values of $C_{L} / A R$. In the present case the relevant $C_{L} / A R$ value for any specific point may be found from Figure $4.7 b$ using the fact that $\lambda=5.375$ in the previous figure. Figure 4.8 presents the results of a limited study (for $C_{L} / A R=0.25$ ) using proper wavelengths for each run. An increase in timeto-converge with increasing flap span is indicated. In view of the significance of other parameters (notably vortex radius), there is a very real question whether it is preferable to treat even twin pair cases on an individual, rather than a parametric basis. 
When convergent vortices reach the center plane, they merge and dissipate. However, it is possible and even probable, in still air, that the surviving vortex will still represent a hazard. Figure 4.9 shows that, for short convergence times near the lower hatched boundary, the surviving vortex is undesirably strong. At any given flap span (>.333b) it is evident that the surviving vortex strength is maximized if flap- and tip-vortex strengths are equal. It is possible to adopt a systems approach to define a minimum hazard condition and then determine optimum flap spans and vortex strengths for a given flight condition with both the surviving vortex strength (Figure 4.9) and time-to-converge (Figure 4.7a) taken into account. However, Figure 4.7a should be revised to include values of spiral wavelength consistent with the relevant $C_{L} / A R$ condition before such studies are undertaken.

\subsection{Three-Dimensional Effects}

As indicated above, changes in spiral wavelengths were found to have a significant effect on time-to-converge. Figure 4.10, which was calculated on the same space-fixed basis as the previous study, shows an increase in timeto-converge as wavelength is increased. When two-dimensional calculations were attempted, to provide a limiting case, convergence was not obtained even well beyond $\tau$ values corresponding to the six-mile limit generally applied. This indicates agreement between the present numerical result and Bilanin's (ref. 6) analytical result which predicts infinite time for two-dimensional cases outside the lower boundary.

The detailed reasons for wavelength dependence are not understood. However, it is noted that the self-convection effect on an element is similar in nature in a spiral to that found previously for plane waves; there is a tendency to slow down the gross rotation rate. Another distinction concerns "axial pumping." A tightly-wound spiral vortex has transverse vorticity components, which correspond to "ring" vorticity. In the core of the spiral, these vorticity components induce a velocity away from the generating wing and a proportion is felt by the spiral itself, tending to lengthen it. It follows that any vortex spiral geometry generated on the basis of quasi-two-dimensional assumptions will have too short a wavelength. Figure 4.11 quantifies this effect. Vortex spirals were first calculated two-dimensionally, then relaxed three-dimensionally in a wing-fixed frame of reference. In view of the $\lambda$ dependence of time-to-converge (Figure 4.10 ), it is clearly desirable to determine a 3-D relaxed wavelength, rather than one derived simply from twodimensional considerations, before embarking on detalled or definitive studies.

\subsection{Notes on Computing}

Initialization. We saw in the preceding section that a spiral wavelength assigned on the basis of two-dimensional considerations [Eqns. (19) and (20)] will not be in equilibrium axially. It has also been necessary to assign unit 
value to the tip vortex span, in the interest of reducing the number of variables. To circumvent such difficulties, it is now clear that any space-fixed calculation involving more than one vortex pair should at least start with a corresponding aircraft-fixed calculation which determines vortex spans and wavelengths, which are in self-equilibrium and which are consistent with the span loading. The two-dimensions-plus-time type of calculation provides a suitable starting geometry for three-dimensional relaxation of an appropriate (i.e. more than one-full wavelength) length of wake.

Since it is unfeasible to relax the equivalent of several miles of wake simultaneously, in aircraft-fixed axes, a transition to a single-wave, spacefixed calculation is made. A suitable whole wavelength is selected* which is downstream of the direct influence of the wing; this is assigned time value $\tau=0$ in the space-fixed calculation. Figure 4.12 shows how subsequent time steps march down the wake. There are, thus, three computational components to the distance of any particular point from the wing. These are: $x_{1}$, the distance from the wing to the leading edge of the frame $\tau=0$; $\tau$, which defines the distance travelled by the leading edge of the frame; and finally $x_{0}$, the axial distance within the frame $\tau$, measured from its leading edge.

The trajectory of a particular point. Since the point which converges is of predominant interest in the present study, we shall use this as an example. At the start of the calculation, it cannot be known which point will converge. Consequently, all points on every frame must be output. Once the convergent point has been identified, however, its complete trajectory may be determined, frame-by-frame, back to the point $x_{0}$ on the initial frame. The examples in Figure 4.5 were plotted in this way. $\tau_{0}$, which corresponds to the position on the original frame, is given by

$$
\tau_{0}=\frac{2 N-1}{\text { NTOT }} 2 \lambda
$$

where $\mathrm{N}$ is the vortex element number and NTOT is the number of elements per wave. The total time-to-converge is thus given by

$$
\tau_{c c}=\tau_{1}+\tau_{0}+\tau_{c}
$$

where ' $\tau_{1}$ equals $x_{1} / U_{\infty}$ and $\tau_{c}$, as before, is the time step (space fixed) at which convergence occurred. Any other specific point may be treated in the same way.

Conjugate runs. In twin-pair parametric studies, advantage may be taken of the fact that, after the first half wavelength, the flap and tip vortices interchange. If these are now renamed and suitable changes are made to the length scales, a new point on the parametric may may be obtained. If a tilde is used to signify the conjugate run, we may write

$$
\tilde{\gamma}=1 / \gamma
$$

*For multiple-pair cases, see Section 5. 
and

$$
\frac{\tilde{n}_{F}}{\tilde{n} T}=\frac{{ }^{2 n_{C G}}-n_{T}}{{ }^{2 n_{C G}}-n_{F}}
$$

The assumption implicit above, that true spiral form is retained for the first half wave, has been confirmed in practice. However, attempts to carry out a similar procedure further down the wake have shown that true conjugates are not obtained; distortion of the spiral has become too significant.

Size of time-step. The angle, $\Delta \theta$, through which the joining line between two vortices rotates during one time step, $\Delta \tau$, is given approximately by:

$$
\Delta \theta=\frac{\left(\Gamma_{1}^{\prime}+\Gamma_{2}{ }^{\prime}\right)}{2 \pi\left(n_{T}-n_{F}\right)^{2}} \Delta \tau \text { radians }
$$

For consistent accuracy, it is fairly obvious that the value of the stepping index, $\Delta \theta$, must be maintained at an appropriately small level for all cases. it is also evident that as $n_{F}$ or loading becomes large, a large number of small time steps will be required. Typical step sizes, $\Delta \tau$, in the previous study have ranged from $1 / 3$ to 2.0 , depending upon flap span, at a $\left(C_{L} / A R\right)$ value of 0.1714 . It is generally found that the longer running cases (larger $n_{F}$ ) also require the smaller values of time-step. This affects computation time severely..

Figure 4.13 shows the effect of step size on time-to-converge for four flap and tip vortex geometries. Quite good accuracy is obtained provided the stepping index is maintained at 8 -degrees or below. Thereafter, the larger $n_{F}$ cases degrade fairly rapidly. This occurs not only because longer times result at larger $\overline{n_{F}}$ but also because errors are larger at any given step size. Figure 4.14, which shows the relative distance between two vortices after 50 time units, demonstrates this. In the absence of center-plane images, an ideal stepping system would maintain constant spacing indefinitely. Figure 4.14 shows that deviations from this trend increase with $n_{F}$. 


\section{MULTIPLE-PAIR CALCULATIONS}

\subsection{Introduction}

For each vortex pair in a multi-component system, there are at least three independent variables, even if a horizontal, unswept trailing edge is assumed. These are: vortex strength, span position, and core radius. Effective viscosity is a possible fourth candidate. For $\mathrm{N}$ multiple vortices there are thus $3 \mathrm{~N}$ or $4 \mathrm{~N}$ minus one variables. (Length scale may be embedded in the core Reynolds number.) For a twin vortex system, a two variable map like Figure 4.7 must be repeated at least over a parametric range of $C_{L} / A R$, for example, even for constant core properties.

It is evident that it is not profitable to attempt to extend generalized results beyond twin-pair cases. A number of realistic, multiple-pair cases have therefore been selected, as examples for the present section, involving up to seven vortex pairs. These cases, defined in Figure 5.1, will be described further in later sections.

An additional motivation for treating cases individually concerns the cálculation itself. For example, if a 4-pair rather than a two-pair case is considered, point density must be increased along each vortex, by a factor of two, giving four times as many points and a factor of 16 on the number of velocity calculations required. Yet a further factor of two is-needed to properly relate the time step-size to vortex spacing*, yielding an overall computation increase of 32-times, or $2^{5}$. Computer run time considerations are much more critical than core storage requirements, which vary only as the square of the number of vortex pairs.

For these reasons, and because of the relative cost-per calculation, three-pair runs and up were carried out on Lockheed-Georgia computers only as necessary for program checkout, i.e. one or two time steps. The multiple pair runs reported in this section were made on the NASA-Ames CDC 7600 computer during a three-week period which included program checkout. Part of the development of the aircraft-fixed algorithms was also carried out at this time. This was sufficient to complete a number of test cases and to establish preferred methods and guidelines. Owing to the "one-shot" nature of the runs it was not possible, however, to produce completed calculations which may be regarded as totally definitive. The Ames results, plus subsequent studies, indicate that the program does have the required capability, however.

*This is mandatory for aircraft-fixed calculations, but some relief is possible in space-fixed cases. 


\subsection{General Approach}

The decay of a complex, multi-vortex wake involves successive reductions in the number of vortices, either via convergence at the center plane (see Section 4 ) or by merging of two vortices into one (see ref. 10). Figure 5.2 shows how the length of the wake may be partitioned on this basis. In the present context, the terms "near-," "middle-," or "far-wake" relate only to the number of vortices present and have no specific counterparts in miles.

Starting at the downstream end, we see that the "far" wake contains just one vortex pair. Since this has the potential for very long life under calm conditions, it is important to design the initial wake configuration in such a way that this remaining pair is a weak one. (We saw in Section 4 that, with suitable starting conditions, earlier convergence of stronger pairs is possible).

The "middle" wake involves a twin pair. This may be dealt with either using parametric plots or on a specific basis depending upon whether the geometry is near-spiral or has suffered noticeable distortion in the near wake*. In the latter case, space-fixed calculation methods may be used, as described in Section 4.

It is the "near" wake which is of principal concern in this section. Considering briefly the simplest, three-vortex case, it is noted that an embedded twin-pair wavelength will no longer characterize the motion. In most cases, this will prevent effective use of a space-fixed analysis, because the wavelength over which the motion is cyclic (assuming no convergences) is impractically long. It follows that an aircraft-fixed analysis must be used.

The dependence of computer run time upon the fifth power of the number of vortices (see Section $5 . \overline{1}$ ) makes it highly desirable to el iminace from the calculation those vortices which converge early and to merge those which spiral together in close proximity. Figure 5.3 shows examples of both types. This particular case concerns a "sawtooth" loading configuration designed by Rossow (ref. 5). The reduction from seven to four vortex pairs is clearly most desirable, computationally.

The results shown in Figure 5.3 were obtained using an aircraft-fixed two-dimensional calculation. A corresponding three-dimensional relaxation was attempted but proved unstable. Since complicated 3-D relaxation cases are likely to require individual treatment to overcome stability problems and are liable to be very expensive to run, it may be acceptable to rely on a simpler method for the early wake provided that the convergence or merging events are strongly indicated. If this is so, the predictive accuracy of the overall calculation will probably be acceptable.

*If specific core details are to be used, the parametric approach is likely to be precluded. 
In summary, the overall approach includes the following steps, given vortex dispositions along the trailing edge and their strengths:

(1a) Use 2-D, reference 9, or (preferably, if stable) 3-D relaxation methods to identify and treat each vortex merging and convergence to the center plane. This will usually reduce the wake to no more than three or four vortex pairs.

(1b) Continue to treat the remaining wake using aircraft-fixed methods until two pairs remain.

(2) If the remaining twin pair is largely undistorted and individual core details are not available, a parametric map may be consulted. A recommendation for the preparation of such plots is included later in this report.

(3) Once one member of the twin pair has either converged on the center plane or merged with its mate, the ensuing single pair wake cannot be calculated by present methods because it is straight and undisturbed in still air. If it is possible to postulate a periodic disturbance, the methods of Section 3 may be used.

\subsection{Three-Dimensional Relaxation of the Near Wake}

- Before starting a three-dimensional relaxation, it is desirable to determine a reasonably close approximation to the final result. This reduces computer time and may prevent instability problems in some cases. Either a two-dimensional or a quasi-two-dimensional (ref. 9) initial run may be used. Figure 5.4 shows an example of each type, together with a fully-relaxed, three dimensional run for the same case. Both of the two-dimensional methods display wake geometries very similar to the fully-relaxed, three-dimensional method. The fully-relaxed run does not extend as far downstream as the two-dimensional runs due to computer limitations.

The three-dimensional relaxation process is well behaved for all the three-pair wakes reviewed. Disturbances originating near the trailing edge propagate away at approximately mainstream speed (see Figure 5.5) leaving a stable vortex system whose transverse velocities are of order $10^{-5}$ or $10^{-6}$ the limit of computer resolution. The vertical arrows in Figure 5.5 depict the downstream end of the converged region.

Attempts were made to converge 4-, 5-, 6-, and 7-pair cases in the same manner as above, with decreasing degrees of success. However, all of these cases employed a swept trailing edge starting line which caused elements on adjacent vortices to be staggered relative to each other. It is believed that this destabilized the relaxation calculation. 


\subsection{Three-Pair Cases}

Certain three-pair wakes may be described as near-cyclic [see Figure 5.6(a)], while in others [Figure 5.6(b)] the third vortex causes irregularities in the shorter wavelength which gives the overall motion a more transient nature. In such cases, there is, therefore, no prospect for using a spacefixed analysis which uses reflection techriques based on the shortest. wavelength.

In near-cyclic cases [Figure 5.6(a)], an attempt was made to simplify the problem by assuming that beyond the initial relaxation region, the third vortex remains straight:* A space-fixed calculation at the shorter wavelength was then carried out with the full streamwise image system, as used previously for twin pairs. As for the corresponding aircraft-fixed case, the innermost vortices travelled together as they were convected downward and outward by the main flap vortex. However, a rapid spiraling then occurred which is uncharacteristic of the aircraft-fixed result. The straight-vortex approximation was therefore unsuccessful.

Recognizing the inherent aircraft-fixed nature of the expected phenomena, the above data were scanned as if by an observer moving at aircraft speed. This produced improvements in some cases, but cyclic instabilities which occurred at frame passage frequency prevented useful results from being obtained. However, deeper investigation suggested a hybrid aircraft-fixed/ space-fixed calculation technique which appears promising. This will be described in Section 5.6 .

\section{5 "Jumbo-Jet" and "Sawtooth" Cases}

The Boeing 747 runs outlined in Figure 5.1 were selected in the light of recent wind tunnel and flight wake hazard test experience at NASA concerning the effect of removing the outer flap and using the inner flap alone to restructure the vortex wake, and concerning the effect of landing gear deployment on such results. In the present calculations the representation of the gear is via its estimated effect upon span load: this omits all viscous wake considerations. Nonetheless, the gear-related span-load changes are found to have a significant impact upon the vortex wake structure. Pre-runs, to identify convergence, merging, etc. (see Section 5.2), permitted simplifications to the distributions shown in the upper part of Figure 5.1 such that three-pair loading configurations could be used as depicted in the lower part of that figure. Three-dimensional relaxation of these three-pair configurations to a steady state then produced the wake structures shown in Figure 5.7 .

The upper two cases of Figure 5.7 correspond to early flight tests which were carried out, gear-up, for full flaps and for inboard flaps only. Wider vortex dispersion is evident in the latter case, which should be beneficial. Lowering the gear reduced the strength of the (anti-lift) inboard flap vortex in both cases. With outer flaps present, lowering the gear caused a much less regular periodic near-wake solution. The reduction in inboard vortex

*Though not fixed. 
strength, due to the gear, also greatly reduced the tendency of the flap and tip vortices to spread apart. A more hazardous wake is therefore implied with the gear down. This corresponds to flight test observations.

The results of Figure 5.7, and other "jumbo"-jet runs not reported here because of step-size problems, have not been extended to determine time-toconverge. Two-dimensional extensions of the B747 runs have been made which indicate that convergence is occurring in the cases shown, but such runs can give no further information because curvature terms, which lead to convergence, are lacking.

It is urged that the "jumbo"-jet demonstration runs started during the present study, are completed and compared with corresponding flight and wind tunnel test results. The use of the sliding-frame calculation method (see below) should make such studies more economical than was envisaged previously.

\subsection{The Sliding-Frame, Aircraft-Fixed Calculation Method}

In previous sections of this report, space-fixed and aircraft-fixed calculations have been regarded as quite separate approaches for solving the far vortex wake. However, a review of results, particularly the moving observer, space-fixed study of Section 5.4, has suggested a hybrid method which is best regarded from an aircraft-fixed point of view.

In Figure 5.5, the history of an aircraft-fixed three-dimensional relaxation is dominated by a disturbance wave which propagates downstrean at approximately mainstream speed and leaves behind the desired steady solution. Let us define the wave propagation speed as $\beta$ times aircraft speed. We may choose a time, in the aircraft-fixed calculation, at which direct wing influence is no longer felt at the position of the disturbance front: "TIME $=20$ " is suitable (Figure 5.5). A calculation frame, stretching, say from Station 16 to Station 28, is now drawn around the disturbance front. If this frame is slid along the wake at $\beta$ times mainstream speed, the disturbance front in Figure 5.5 remains centered and converged wake is always contained in the left half of the frame. If suitable precautions are taken with conditions at the ends of the frame, particularly upstream, this provides an attractive and economical basis for a wake solution. In fact, if $\beta=1$, the method reduces to a spacefixed calculation. The corresponding $(\beta=1)$ program changes are trivial.

Treatment of the downstream end of the frame presents little difficulty: a two-dimensional type of initial solution will clearly suffice, as for the aircraft fixed case. At the upstream end care must be taken both to ensure that a sufficient length of converged wake is included and to provide sufficiently realistic upstream conditions that the converged wake within the box is not contaminated. Such questions could be resolved by comparison with existing cases run with the current aircraft-fixed algorithms.

Since the program changes involved are minor, and test examples exist, the above modifications and checkout appear to be highly worthwhile. 


\section{CONCLUSIONSS AND RECOMMENDATIOINS}

A computer program has been developed which uses finite-element threedimensional relaxation methods to calculate the development of vortex wakes behind aircraft for a considerable downstream distance. The program is now operational at NASA-Ames. The inclusion of a self-induction term in the solution, dependent upon local curvature and vortex core radius, permits calculation of finite lifetimes for systems for which infinite life would be predicted two dimensionally. The present report describes the program together with single-pair, twin-pair, and multiple-pair studies carried out using it. Though "jumbo" aircraft wake examples are included, these have not been carried far enough for other than qualitative conclusions to be drawn.

The wake studies have lead to the following conclusions:

Single-Pair Wakes

(1) There is a lower limit to the wavelengths at which the "Crow"-type instability can occur. Below this limit, self-induction effects cause the plane of the disturbance waves to rotate counter to the vortex direction.

(2) Disturbance waves in a horizontal plane are several times more stable than the classic, 45-degree "Crow" case. Vertical and spiral waves are slightly more stable.

Twin-Pair Wakes

(3) There is an important distinction between single-and twin-pair vortex convergence at the center plane. The first is usually a space-fixed event, but the latter moves at aircraft speed.

(4) The self-induction term causes the time taken for vortices to converge at the center plane to be a strong function of wavelength (see Figure 4.10). Either vortex can converge first (Figure 4.6).

(5) In the limited twin-pair studies performed for constant $C_{L} / A R$, time-to-converge increases with increasing flap span.

(6) Choosing equal strengths for flap (for $\eta_{F}>333$ ) and tip vortices maximizes the strength of the vortex which survives after one has converged at the center plane (see Figure 4.9).

\section{Multiple-Pair Wakes}

(7) Though space-fixed axial-imaging techniques can be used for singleand twin-pair wakes, they are not applicable directly to multiple pairs. 
Calculations must at least start in an aircraft-fixed coordinate system. Thereafter, a "sliding-frame" method is suggested which has potential for applying three-dimensional relaxation methods economically to the far wake. The method is very similar to a space-fixed calculation but is controlled differentiy (see Section 5.6).

(8) Pilot studies of Boeing 747 configurations show correct qualitative response to removal of the outer flap and to gear deployment, as compared with wind tunnel and flight test experience (see Figure 5.7).

\section{Recommendations}

(1) More detailed studies should be undertaken of the separate effects of vortex core radius and relaxed wavelength on the time to attain centerplane convergence in twin-pair cases. This should provide useful design guidance for low-hazard wakes.

(2) Further time-to-converge, twin-pair parametric studies (see Section 4.2) should be carried out, holding $C_{L} / A R$ constant for each of several maps like Figure $4.7 \mathrm{a}$ and 4.8 .

(3) The "sliding frame" calcultion method should be implemented and used to complete the present jumbo-jet studies.

(4) More comprehensive studies should be made concerning wakes designed for early disruption, e.g. the "sawtooth" design. This requires more detailed aircraft fixed runs for near wake, including an improved rationale for vortex merging. If significant vortices then remain, the "sliding frame" method should d-be applied. 


\section{REFERENCES}

1. Crow, S. C.: Stability Theory for a Pair of Trailing Vortices. AIAA Joumal, vol. 8, no. 12, December 1970, pp. 2172-2179.

2. Hackett, J.E.; and Theisen, J. G.: Vortex Wake Development and Aircraft Dynamics. Aircraft Wake TurbuZence and Its Detection. Edited by J. Olsen, A. Goldberg, and M. Rogers, Plenum Press, 1971.

3. Chevalier, H.: Flight Test Studies of the Formation and Dissipation of Trailing Vortices. J. Aireraft, vol. 10, no. 1, January 1973, pp. 14-18.

4. Widnall, S. E.; Bliss, D. B.: and Zalay, A.: Theoretical and Experimental Study of the Stability of a Vortex Pair. Aircraft Wake Turbulence and Its Detection, edited by J. Olsen, A. Goldberg, and M. Rogers, Plenum Press, 1971.

5. Rossow, V. J.: Theoretical Study of Lift-Generated Vortex Wakes Designed to Avoid Roll-Up. AIAA Journat, vol. 13, no. 4, April 1975, pp. 476-484.

6. Bilanin, A. J.; Donaldson, C. duP., and Snedeker, R. S.: An Analytical and Experimental Investigation of the Wakes Behind Flapped and Unflapped Wings. AFFDL-TR-74-90, May 1974.

7. Lamb, Sir Horace: Hydrodynamics. Sixth ed. Dover Publications, 1945.

8. Leonard, A.: Numerical Simulation of Interacting, Three-Dimensional Vortex Filaments. Proc. of the Fourth International Conference on Numerical Methods in Fluid Dynamics, Spring-Verlag, 1974.

9. Hackett, J. E.; and Evans, M. R.: Vortex Wakes Behind High-Lift Wings. J. Aircraft, vol. 8, no. 5, May 1971, pp. 334-340.

10. Rossow, V. J.: Convective Merging of Vortex Cores in Lift-Generated Wakes. AIAA Paper No. 76-415.

11. Proceedings, NASA Symposium on Wake Vortex Minimization, Washington, February 25-26, 1976. 
APPENDIX

VORTEX LOOPING COMPUTER PROGRAM OPERATION

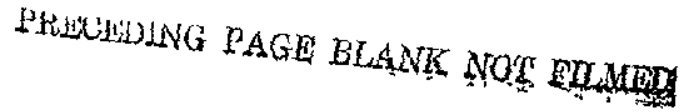




\section{GENERAL PROGRAM OPERATION}

A computer program has been developed to apply the previous methods to multiple vortex pair instabilities. The program has been implemented on the U1106 computer and the MAC-16 mini-computer at Lockheed-Georgia and the IBM 360-67 and the CDC-7600 computers at NASA-Ames.

The program has the capability to analyze up to seven vortex pairs and up to ninety points per wavelength in the cyclic, space fixed option and ninety points per vortex in the aircraft-fixed option. In the space-fixed option each point is perturbed at each time step and a new geometry is calculated for the next time step. Since the frame of reference is fixed in space, no velocity is imparted to the system except that induced on each point by the system. The calculations are continued until the vortex system becomes unstable.

The aircraft-fixed option is more complex. An initial two-dimensional time dependent, space-fixed calculation is performed. Time is then converted to downstream distance to set up the initial geometry for a three-dimensional time relaxation. In the aircraft-fixed frame of reference the free stream velocity must be added to the induced velocities. The free stream velocity causes the points to move downstream. To keep the frame of reference fixed with the aircraft, the points must be reordered at each time step. Each point, $N$, at time, $t$, becomes the point, $N+1$, at time, $t+\Delta t$. The points on the wing are fixed with time, and the trailing edge point is used twice at $t+\Delta t$; once fixed on the wing and once shed downstream. The last downstream point is removed at each time step. The calculation continues until equilibrium is reached. This normally requires that the number of time steps equal the number of points per vortex considered.

\section{INPUT}

The general input parameters to the vortex looping program are:

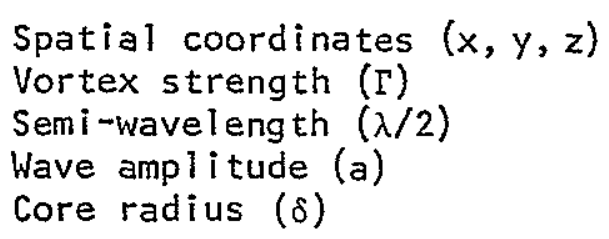

A description of the input parameters is given in Täble $A-1$. Allvariables used in the vortex looping program are normalized on wing semispan (b/2) and free stream velocity $\left(U_{\infty}\right)$. If the subscript, $s$, denotes normalized value used in the program and the subscript, $o$, denotes dimensional values, then: 


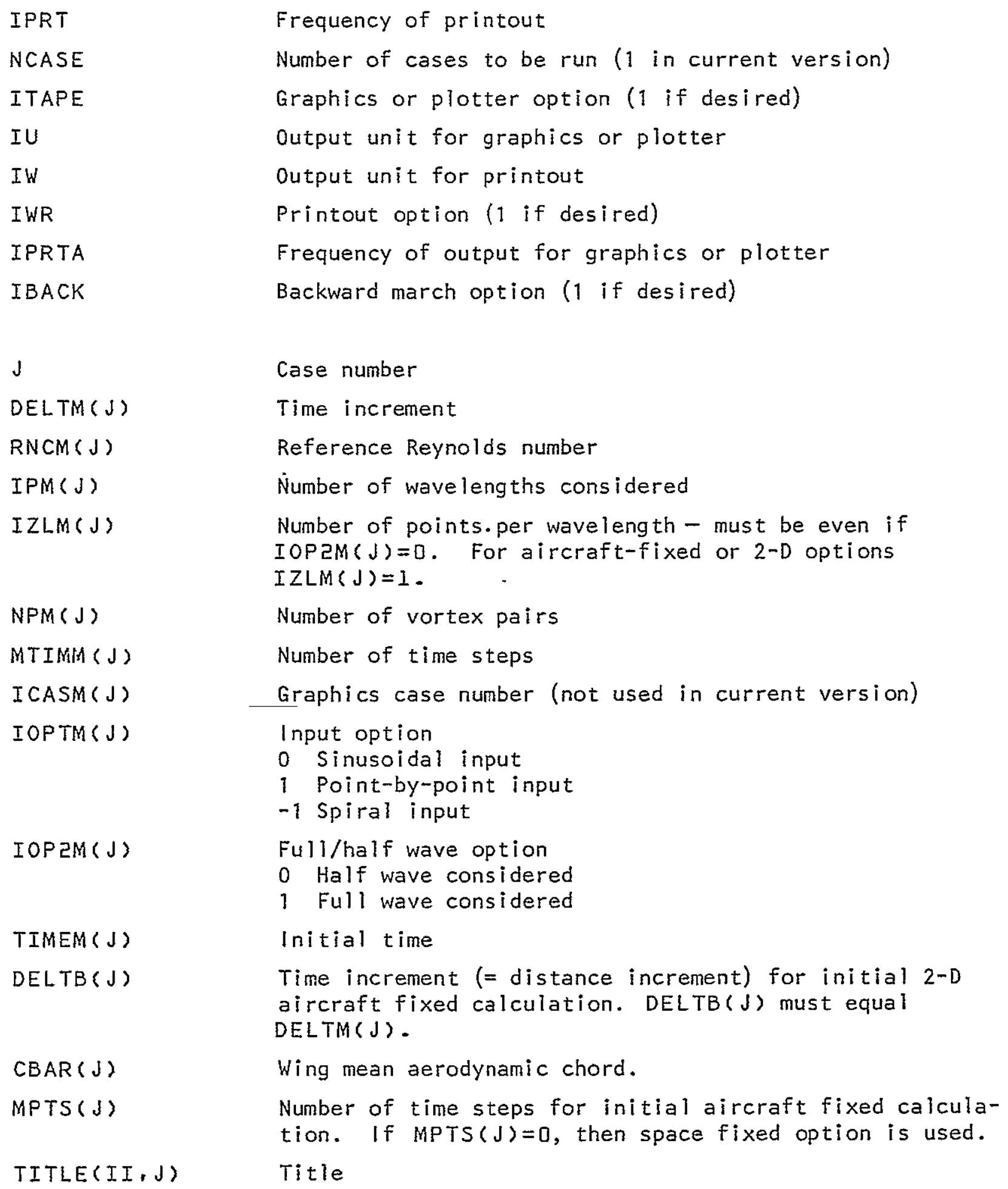


TABLE A-1. INPUT PARAMETERS (Concluded)

I

$\operatorname{NPAM}(I, J)$

$\operatorname{CIRCM}(I, J)$

$W V L M(I, J)$

$\operatorname{AMPXM}(I, J)$

$\operatorname{AMPYM}(I, J)$

$\operatorname{RCORM}(I, J)$

$\operatorname{sSM}(I, J)$.

$H I N M(I, J)$

PHASM(I,J)

$\mathrm{N}$

$X I N(N, I, J)$

$Y I N(N, I, J)$

$\operatorname{ZIN}(N, I, J)$

$\operatorname{RCOMI}(N, I, J)$
Vortex pair number. For aircraft fixed calculations, vortices must be input from inboard to outboard.

Vortex reference number

Vortex strength

Wavelength/2.0. Must be the same for all vortices.

Amplitude of sinusoidal/spiral disturbance in $x$-direction

Amplitude of sinusoidal/sprial disturbance in y-direction

Core radius.

Initial spanwise location of vortex

Initial vertical location of vortex

Phase shift of disturbance. Must be the same for all vortices.

Point number for point-by-point input

$X$ location of $N$

$Y$ location of $N$

$Z$ location of $N$

Core radius of $\mathrm{N}$ 


$$
\begin{aligned}
x_{i_{s}} & =x_{i_{0}} /(b / 2) \\
\Gamma_{s} & =\Gamma_{0} /\left(u_{\infty} b / 2\right) \\
v_{i_{s}} & =v_{i_{0}} /\left(u_{\infty}\right) \\
t_{s} & =t_{0}\left(u_{\infty} / b / 2\right) \\
\lambda_{s} & =\lambda_{0} /(b / 2) \\
a_{s} & =a_{0} /(b / 2) \\
\delta_{s} & =\delta_{0} /(b / 2)
\end{aligned}
$$

The input format is summarized in Table A-2. Note that $x$ is the spanwise coordinate, $y$ is the vertical coordinate, and $z$ is the downstream coordinate.

\section{MAIN PROGRAM/SUBROUTINE FUNCTIONS}

The principal functions performed by the main program and each subroutine are:

SUBROUTINE

Main Program

\section{FUNCTIONS}

1. Case data input.

2. Initial geometry for one side of each vortex pair determined using sinusoidal or spiral distribution or point-by-point input.

3. Other parameters used in calculations determined $\left(x_{i}\right.$ DUM, $C x_{i}=\Gamma \Delta x_{i}$, etc. $)$.

4. Displacements and new geometry for each time step calculated.

5. Geometry at each time step output.

1. Induced velocities summed.

2. Element compression applied. 
TABLE A-2. INPUT FORMAT

\begin{tabular}{|c|c|c|}
\hline Card & Format & Input Parameters \\
\hline 1 & 914 & $\begin{array}{l}\text { IPRT, NCASE, ITAPE, IU, IW, IWR, IPRTA, } \\
\text { IBACK }\end{array}$ \\
\hline 2 & $2 F 10.0 .794$ & $\begin{array}{l}\operatorname{DELTM}(J), \operatorname{RNCM}(J), \operatorname{IPM}(J), \operatorname{IZLM}(J), \operatorname{NPM}(J) \\
\operatorname{MTIMM}(J), \operatorname{ICASM}(J), \operatorname{IOPTM}(J), \operatorname{IOP} \operatorname{IOM}(J)\end{array}$ \\
\hline 3 & $\exists F 10.0 .14$ & $\begin{array}{l}\operatorname{TIMEM}(J), \operatorname{DELTB}(\mathrm{J}), \operatorname{CBAR}(\mathrm{J}), \operatorname{MPTS}(\mathrm{J}) \\
\text { (If aircraft fixed calculations are not to be done, } \\
\text { then MPTS }(J)=0 .)\end{array}$ \\
\hline 4 & $20 A 4$ & $\operatorname{TITLE}(I I, j)$ \\
\hline
\end{tabular}

If $\operatorname{IOPTM}(J)=0$

$5 \quad I 4,8 F 9.0 \quad \operatorname{NPAM}(I, J), \operatorname{CIRCM}(I, J), W V L M(I, J), \operatorname{AMPXM}(I, J)$, $\operatorname{AMPYM}(I, J), \operatorname{RCORM}(I, J), \operatorname{SSM}(I, J), \operatorname{PHASM}(I, J)$, $\operatorname{HINM}(I, J)$

If $\operatorname{IOPTM}(J)=1$

5.

$I 4,8 F 9.0$

$\operatorname{NPAM}(I, J), \operatorname{CIRCM}(I, J), \operatorname{WVLM}(I, j)$

6

$4 F 10.0$

$\operatorname{XIN}(N, I, J), \operatorname{YIN}(N, I, J), \operatorname{ZIN}(N, I, J)$, $R \operatorname{COMI}(N, I, J)$

(Repeat card 6 for each point $(N=1$, INPI) on a single vortex pair (one side only).)

(Note: Calculations are performed on the points $N=2$, INPI-1.)

(Repeat cards 5 and 6 for each vortex pair.) 
FAAVEL

1. Induced velocities from each point determıned.

2. Imaging techniques used to determine the effects of up and downstream points and the vortices shed from the other wing.

CURV

1. Local radius of curvature calculated.

2. Core radius at time, $t$, calculated.

3. Self-induced velocity calculated.

STAT

1. Final geometry at end of backward march compared to initial geometry.

\section{PROGRAM LISTING}

A listing of the vortex looping program as used on the U1106 computer follows. 


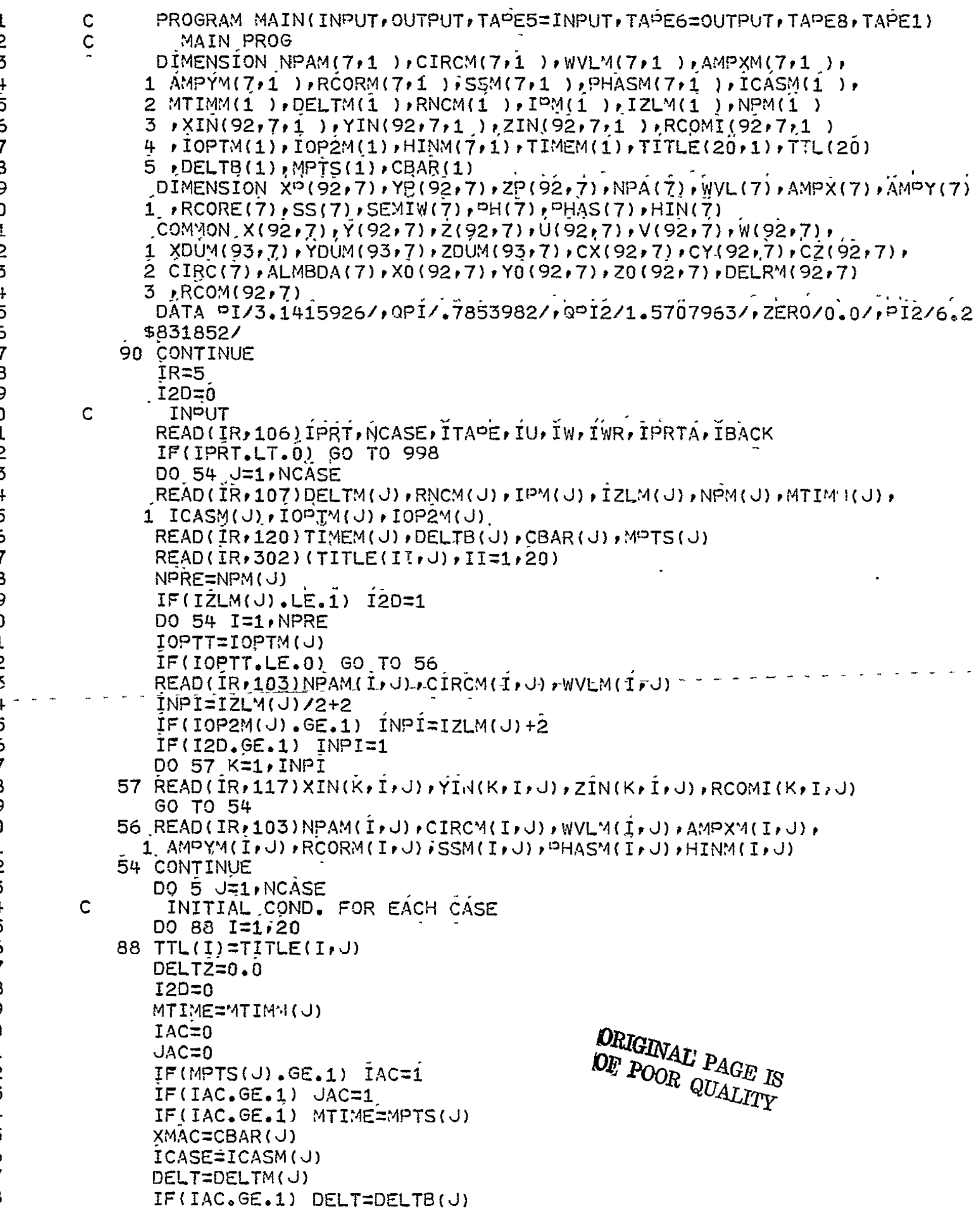




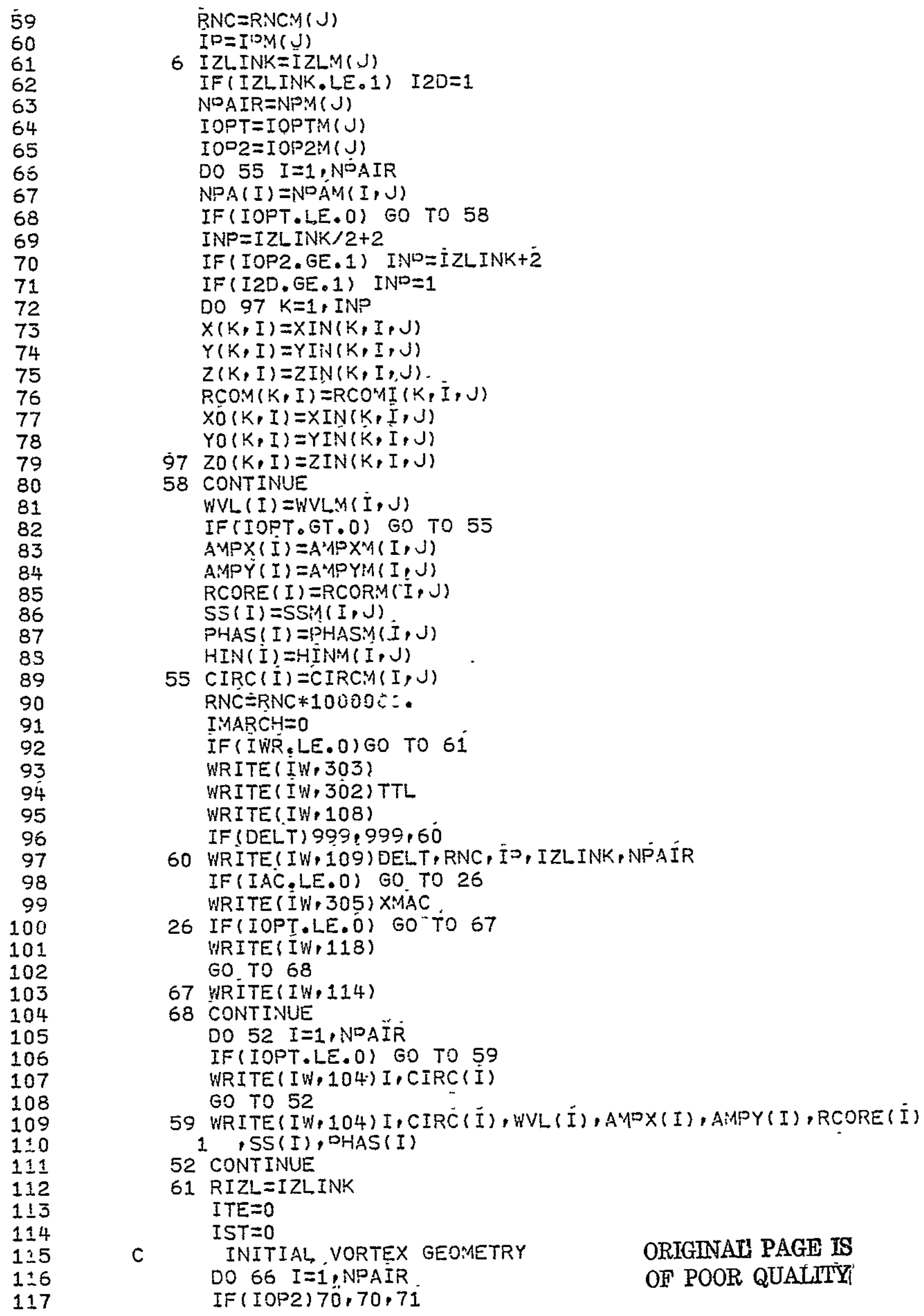

59

60

61

62

63

64

65

65

67

68

69

70

71

72

73

74

75

76

77

78

79

80

81

82

83

84

85

86

87

89

89

90

91

92

93

94

95

96

97

98

99

100

101

102

103

104

105

105

107

108

109

$1 \pm 0$

111

112

113

114

125

$1 \pm 6$

117

BNC=RNCM U)

$I D=I P M(J)$

$6 \operatorname{IZLINK}=I Z L M(J)$

IF (IZLINK.LE。1) I $2 D=1$

NOAIR=NPMA(U)

$I O P T=I D P T M(U)$

$I O D 2=I O P 2 M(J)$

DO 55 I=1,NDAIR

$N P A(I)=N D A M(I, U)$

IF (IOPT.LE. O) GO TO 58

$I N P=I Z L I N K / 2+2$

IF (IOP2.GE.1) IND $=I Z L I N K+2$

IF (I2D.GE.1) IND=1

DO $97 K=1$, INP

$X(K, I)=X I N(K, I, J)$

$Y(K, I)=Y I i N(K, I, J)$

$Z(K, I)=Z I N(K, I, J)$.

$R \operatorname{ROM}(K, I)=\operatorname{RCOMI}(K, I, J)$

$X O(K, I)=X I N(K, I, J)$

$Y O(K, I)=Y I N(K, I, J)$

$97 Z 0(K, I)=Z I N(K, I, J)$

58 CONTINUE

$W V L(I)=W V L M(I, J)$

IF (IOPT.GT.O) GD TO 55

$A M P X(I)=A^{2} 1 P X M(I, J)$

$A M P Y(I)=A \star A P Y M(I ! J)$

$\operatorname{RCORE}(I)=R C O R M(I, U)$

$S S(I)=S \operatorname{Sil}(I, J)$

PHAS (I) $=$ PHASM $(\dot{I}, J)$

$H I N(I)=H I N M(I, J)$

$55 \operatorname{CIRC}(I)=\operatorname{CIRCM}(I, J)$

RNC $=$ RNC $* 10000:$.

IMARCH $=0$

IF (IWR.LE.O)GO TO 61

WRITE (IW, 303)

WRITE (IW, 302) TTL

WRITE $(I W: 108)$

IF (DELT) $999,999,60$

60 WRITE(IW, 109 ) DELT, RNC, ID, IZLINK, NPAIR

IF (IAC,LE.O) GO. TO 26

WRITE (IW, 305) XMAC

26 IF (IOPT.LE.O) GO TOO 67

WRITE (IW, 118)

GO TO 68

67 WRITE (IW, 114)

68 CONTINUE

DO $52 I=1$, NDA $I R$

IF (IOPT.LE.O) GO TO 59

WRITE(IW, 104) I, CIRC (I)

GO TO 52

59 WRITE (IW, 104)I, CIRC (I),WVL(I), AMDX(I), AMPY (I),RCORE(I)

1 ,SS(I), DHAS(I)

52 CONTINUE

61 RIZL $=I Z L I N K$

ITE $=0$

$I S T=0$

C INITIAL VORTEX GEOMETRY

ORIGINATI PAGE IS

DO $66 \quad I=1$,NPAIR.

IF (IOP2) $70.70,71$

OF POOR QUALTIYI 


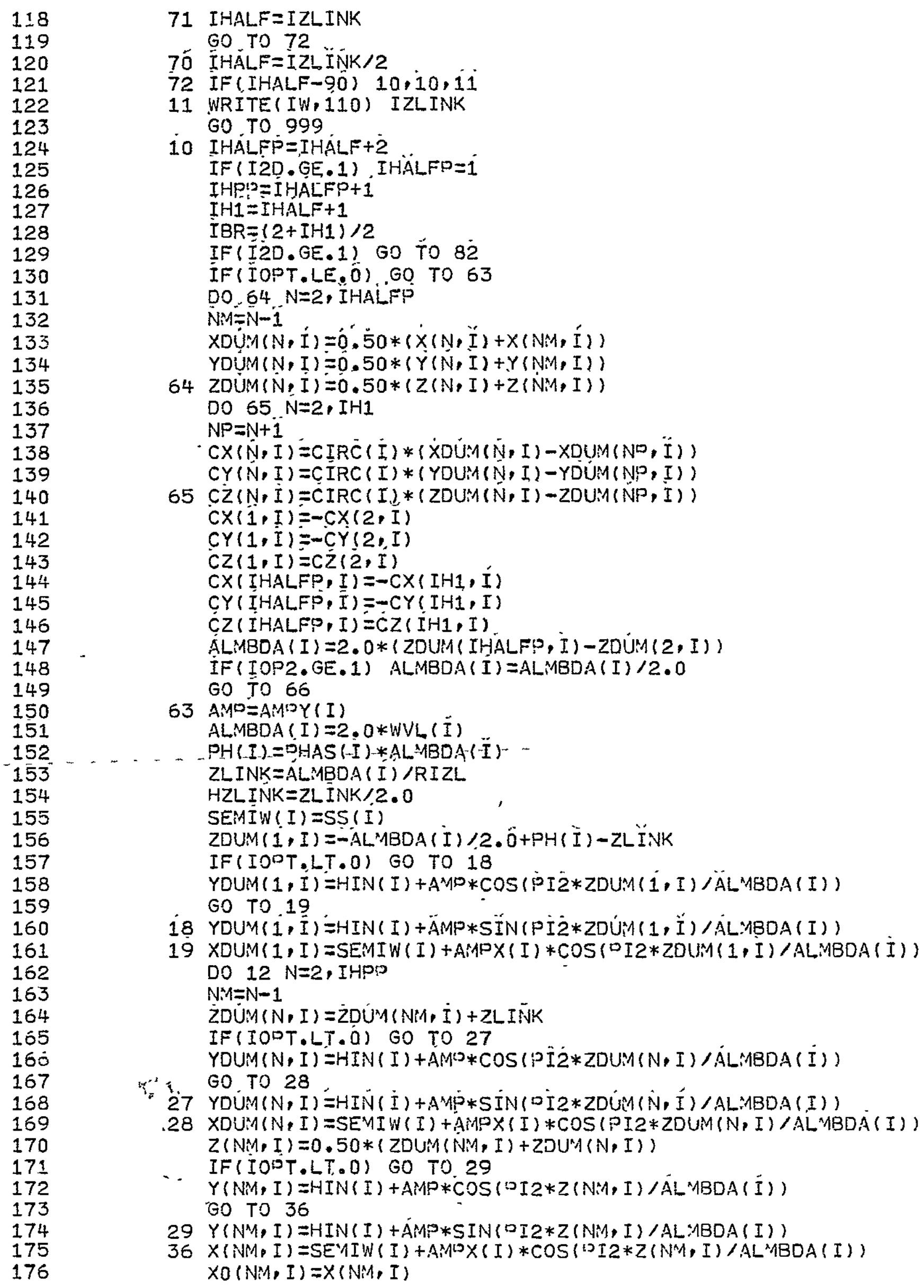

71 IHALF $=I Z L I N K$

GO TO 72

$70 ́$ IHALF $=I Z I O I N K / 2$

72 IF (IHALF-90) $10,10,11$

11 WRITE (IW,110) IZLINK GO TO 999

io IHALFP $=$ IHALF+2 IF ( I $2 D, G E, 1)$ I IHALLF $=1$

IHP'? $=$ IHALFP+1

$I H 1=I H A L F+1$

IBR $\equiv(2+I H 1) / 2$

IF (I2D.GE.1) GO To 82

IF (IOPT.LE.O) .GO TO 63

DO. $64 . \mathrm{N}=2$, IHALF'

$N M=N-1$

$X D U M M(N, \dot{I})=\dot{0}, \dot{5} 0 *(\dot{X}(N, \ddot{I})+X(N M, I))$

$Y D \cup M(N, I)=0.50 *(Y(N, I)+Y(N M, I))$

$64 Z \operatorname{ZUM}(\hat{N}, I)=0.50 *\left(Z(N, I)+Z\left(N_{M} M, I\right)\right)$

DO $65 . \mathrm{N}=2, \mathrm{IH} 1$

$N P=N+1$

- $C X(\ddot{N}, I)=C I R \bar{C}(\dot{I}) *(\dot{X D U M}(\bar{N}, I)-X D U M(N D, \ddot{I}))$

$C Y(N, I)=C \operatorname{IRC}(I) *(Y D U A(N, I)-Y D U M(N P, I))$

$65 C Z(N, I)=C I R C(I) *(Z D U M(N, I)-Z D U M(N P, I))$

$C X(1, I)=-C X(2, I)$

$C Y(1, I)=-C Y(2, I)$

$C Z(1, I)=C Z(2, I)$

$C X(I H A L F P, I)=-C X(I H 1, I)$

$C Y(I H A L F P, I)=-C Y(I H I, I)$

$C Z$ (IHALFO,I) $=\dot{C Z}(I H 1, I)$.

$A L M B D A(I)=2.0 *(Z D U M(I H A L F(I), Z D U M(2, I))$

IF (IOP2.GE.1) ALMBDA (I) $=A L M B D A(I) / 2.0$

GO TO 66

63 AMD $=A M P Y(I)$

ALMBDA (I) $=2.0 * W V L$ (I)

$A L M B D A(I)=2.0 * W V L(I)$
$P H(I)=O H A S(I)+A L M B D A(\ddot{I})$

ZLINK =ALSABDA (I) $/ R I Z L$

HZLINK $=Z L I N K / 2.0$

SEAIW(I) $=S S$ (I)

ZDUM $(I, I)=-A L M B D A(I) / 2.00+P H(I)-Z L I N N K$

IF (IOOT.LT.O) GO TO 18

$\operatorname{YDUM}(1, I)=H I N(I)+A^{M} D * \operatorname{COS}(P I 2 * Z D U M(i, I) / \ddot{A L} M B D A(I))$

GO TO 19

is YDUM(I, I) $=H I N(I)+\ddot{A M P} * S I N(P I 2 * Z D U ̈ 1, \ddot{I}) / A L: M B D A(I))$

$19 \operatorname{XDUM}(1, I)=\operatorname{SEMIW}(I)+A M P X(I) * \operatorname{COS}(D I 2 * Z D U M(1, I) / A L M B D A(I))$

DO $12 \mathrm{~N}=2$, IHP O

$N * 1=N-1$

ZDUMM $(N, I)=Z D U M M(N M, \dot{I})+Z L I N K_{K}$

IF(IODT.LT.QA) GO TO 27

$\operatorname{YDUM}(N, I)=H I N(I)+A M O \operatorname{COS}(P I 2 * Z D U M(N, I) / \dot{A} L M B D A(I))$

Y. GO. TO 28

$27 \operatorname{YDUM}(N, I)=H I N(\dot{I})+A M P * S I N(D I 2 * Z D U M(\dot{N}, \dot{I}) / A L * 1 B D A(I))$

$.28 \times D U M(N, I)=S E M I W(I)+\operatorname{ANPX}(I) * \operatorname{COS}(P I 2 * Z D U M(N, I) / A L M B D A(I))$

$Z(N M, I)=0.50 *\left(Z D U M(N M, I)+Z D U^{M}(N, I)\right)$

IF IIOTT.LT.OO) GO TO. 29

$\because \quad Y(N M, I)=H I N(I)+A M P * \operatorname{COS}(0 I 2 * Z(N * 1, I) / A L M B D A(\bar{I}))$ GO TO 36

$29 Y\left(N^{M}, I\right)=H I N(I)+A M P * S I N(O I 2 * Z(N M, I) / A L: M B D A(I))$

$36 \times(N M, I)=S E M I W(I)+A M(O) X(I) * \operatorname{COS}(0 I 2 * Z(N M, I) / A L M B D A(I))$ $X O(N M, I)=X(N, M, I)$ 
$Y O(N M, I)=Y(N \times 1, \bar{I})$

$Z O(N M, I)=Z(N M, I)$

CX(NM,I) $=C \operatorname{IRC}(I) *(X D U M(N * A, I)-X D U \cup M(N, I))$

$C Y(N M, I)=C I R C(I) *(Y D U M A(N M, I)-Y D U U: A(N, I))$

$12 C Z(N * A, I)=C I R C(I) *(Z D U M(N \wedge, I)-Z D U M(N, I))$

GO TO 65

$82 C X(1, I)=0$.

$C Y(1, I)=0$.

$C Z(1, I)=-C \operatorname{IRC}(I)$

ALMBDA $(I)=2.0 * W V L(I I)$

66 CONTINUE

IF (I2D.GE. I) GO TO 87

$\operatorname{IF}(\operatorname{ID} 2, G E$. 1) DELTZ $=(Z(I H 1,1)+Z(2,1)) / 2.0$

$D E L T Z O=D E L T Z$

CONTINUE

WRITE(IW,111)

$I O T I=0$

IDTA $=0$

TIME $=$ TIMEM ( U)

DO $25 I=1$,NDAIR

- DO $25 \quad N=1$, IHALFP

$X P(N, I)=X(N, I)$

$Y \bar{P}(N, I)=Y(N, I)$

$Z P(N, I)=Z(N, I)$

IF (IOPT.GT.O) GO. TO 25

$R \operatorname{COM}(N, I)=R \operatorname{CDRE}(I)$

25 DELRM $(N, I)=0.0$

$X B A R=0.0$

YBAR $=0.0$.

DELTYB $=0.0$

23 CONTINUE

IF (IAC.LE.O), GO TO 2

$F A C=0.75 * X . M A C / 2.0$

$F A C 1=F A C$

DO $80 \quad I=1$, NDAIR

$K K=1$

$\operatorname{RCOMI}(I, I, J)=\operatorname{RCON}(1, I)$

$Y I N(1, I, J)=Y O(1, I)$

$Z I N(1, I, U)=Z 0(1, I)-2.0$ *FACI

IF(I.EQ.1), GO TO.81

$X I N(1, I, J)=X 0(1, I-1)$

GO TO 80

$81 X I N(1, I, U)=0.0$.

80 WRITE $(I W, 112) K k, X I \dot{N}(1, I, J), Y \dot{I} N(i, \dot{I}, J), Z I N(i, \tilde{I}, J)$

DO 3 I=I,NPAIR

Do $3, K=2,3$

$R \operatorname{COMI}(K, I, J)=R \operatorname{COM}(1, I)$

$X I N(K, I, J)=X O(I, I)$

YIN $(K, I, J)=Y O(1, T)$

ZIN $(K, I, U)=Z 0(1, I)-F A C-F A ̈ C 1$

WRITE $(I N, 112) K, X I N(K, I, J) I Y I N(K, I, J), Z I N(K, I, J)$

3 FAC $=-F A C$

2 CONTTSNUE

DO 50 IT $=1$, MTI I YE

C OUTPUT OF WAVE GEOMETRY

IF (IÁC. GE. 1) $K=I T+3$

IPCO $=0$

IF (IMARCH.GE.1) GO TO 30

IF(IWR.LE.O)GO TO 20 


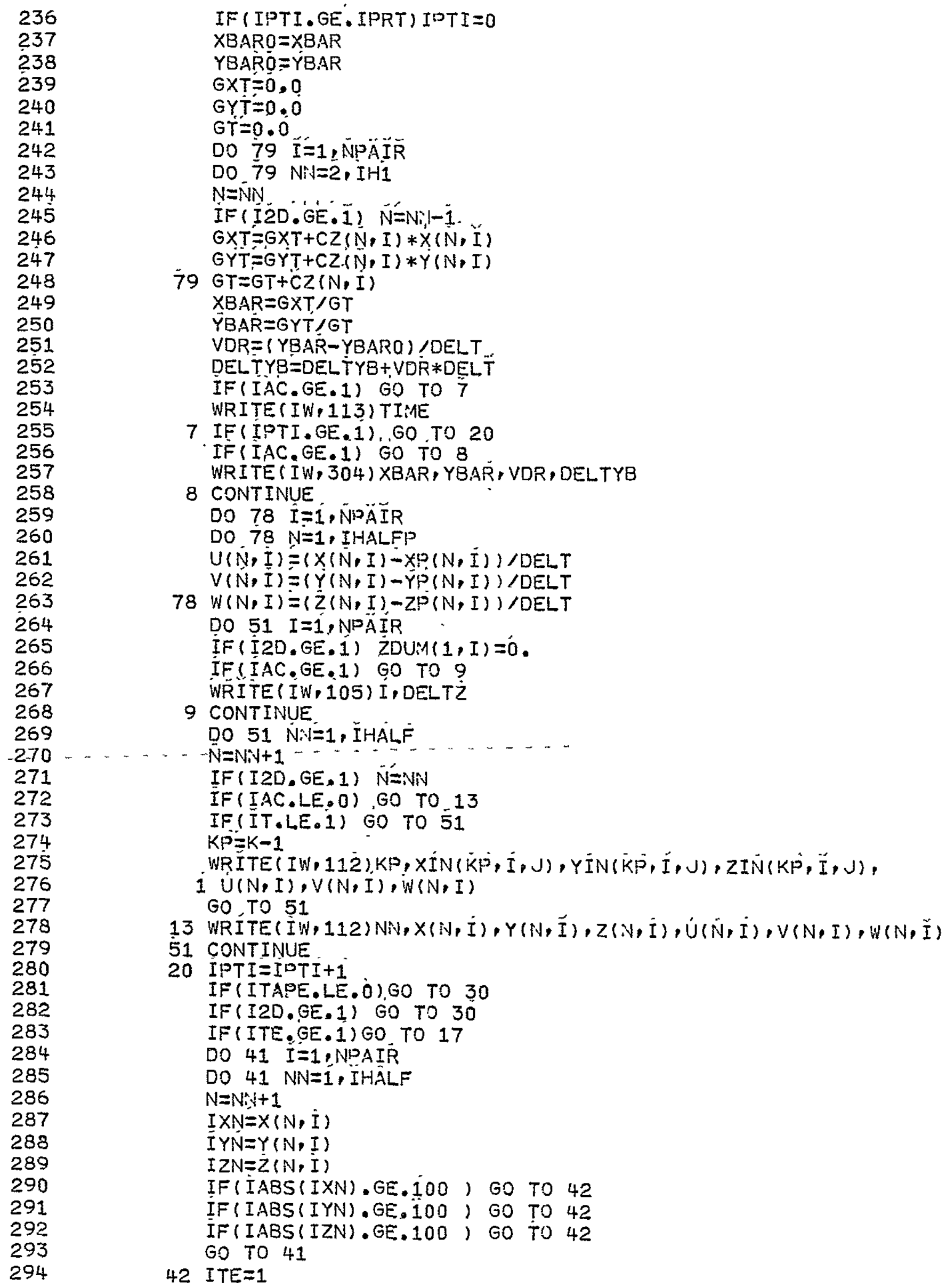

8 CONTINUE

DO $78 \quad \hat{I}=i, N$ NPÖIR

DO $78 \mathrm{~N}=1$, IHALFP

$U(\bar{N}, \bar{I}) \equiv(X(\bar{N}, I)-X P(N, \bar{I})) / D E L T$

$V(N, I)=(Y(N, I)-Y P(N, I)) / D E L T$

$78 W(N, I)=(Z(N, I)-Z P(N, I)) / D E L T$

DO 51 I=1, NPAIR

$\operatorname{IF}(I 20 . G E, 1) \quad Z D U M(1, I)=0$.

IF(IAC.GE.1) GO TO 9

WRITE (IW, 105) I, DELTZ

9 CONTINUE

DO 51 NN $Y=1, \check{I} H A L F$

$-N=N i V+1$

IF (I2D, GE, 1) N̈́NN

IF (I.AC.LE,O), GO TO 13

IF (IT.LE.1) GO TO $\overline{5} 1$

$K P=K-1$

WRITE $(I W, 11 \overline{2}) K P, X I N(\dot{K} P \dot{I}, \dot{I}, J), Y \overline{I N}(\bar{K} P, \dot{I}, J), Z I \bar{N}(K \dot{P}, \ddot{I}, J)$,

$1 U(N, I), V(N, I), W(N, I)$

GO TO 51

13 WRITE $(\dot{I} N, 112) N N, X(N, \dot{I}), Y(N, \tilde{I}), Z(N, \bar{I}), \dot{U}(\tilde{N}, \dot{I}), V(N, I), W(N, \tilde{I})$

51 CONTINUE

20 IPTI I IOTI+1

IF (ITAPE.LE.O) GO TO 30

IF (I2D.GE, 1) GO TO 30

IF (ITE.GE.1) GO TO 17

DO 41 I=1, NPAIR

DO $41 \mathrm{NN}=1$, IHALF

$N=N i+1$

$I X N=X(N, I)$

$\bar{I} Y \bar{N}=Y(N, I)$

$I Z N=Z(N, I)$

IF (IABS(IXN).GE.100) GO TO 42

IF (IABS (IYN).GE,100) GO TO 42

IF (IABS(IZN).GE.100, GO TO 42 GO TO 41

42 ITE $=1$ 
WRITE (IW, 115)

41 CONTINUE

IF (IPTA. GE, IDRTA) IOTA $=0$

IF (IDTA.GE. I) GO TO 17

IF (IT.GT.1) GO TO 86

IF(TIME.GT.0.01) GO TO 17

6 CONTINUE

DO $15 \quad \bar{I}=1$, NDAIR

DO $15 \mathrm{NN}=1$, IHALF

$N=$ iNiv+1

$Y T A P=Y(N, I)-D E L T Y B$

IF (JAC.GE.I) YTAP =Y $(N, I)$

$Z T A P=Z(N, I)-D E L T Z O$

15 WRITE (IU, 300)X(N,I), YTAD, ZTAD

17 IPTA $=$ IOTA+1

30 IF (IT.GE.MTIME) GO TO 50

DO $43 I=1$, NPAIR

DO 43 NiV $=1$, IHALF

$N=i N i v+1$

- IF(I2D.GE.1) N=NN

IF $(A B S(X(N, I))-1000000,144,45,45$

$44 \operatorname{IF}(A B S(Y(N, I))-1030000) 46,45,$.

$46 \operatorname{IF}(A B S(Z(N, I))-1000000) 43,45,$.

45 IST $=1$

WRITE (IW, 116)

GO TO 48

43 CONTINUE

CONTINUE
CALCULATION OF INDUCED VELOCITIES
DO 40 I $=1$, NDAIR

DO, $40 \mathrm{NH}=2$, IHI

$N C=N N$

$\mathrm{N}=\mathrm{NN}$

- IF (I2D, GE, $\overline{1}), \bar{N}=i N-i$

CALL PCOR(N, I, IPCO, IP, IBR, NPAIR, IHI, IHALFP, DELT, IW, IST, IOPẼ, 1 IAC, XYAC)

IF (IST,GE.1) GO TO 48

IF (I2D.GE.1) GO TO 33

IF $(J A C, G E, 1) \quad W(N, I)=W(N, \bar{I})+1: 0$

IF (JAC.LE.O) GO TO 69

IF (N.LE.3) GO TO 83

69 CONTINUE

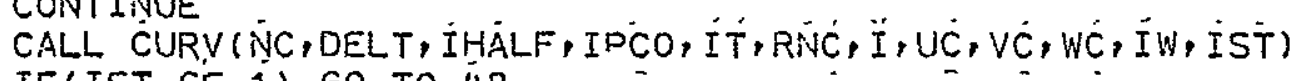

IF (IST,GE.1) GO TO 48

SO. TO 84

$83 \cup C=0$.

$V \dot{C}=0$.

$W C=0$

$84 U(\bar{N}, \bar{I})=U(\bar{N}, I)+U C$

$V(N, I)=V(N, I)+V C$

$W(N, I)=W(N, I)+W C$

40 CONTINUE
MOD. OF "INDUCED VEL. SO THAT VEL ÁLONG VORTEX $=0$

IF (JAC.LE. O) GO TO 96

IF (IAC.GE, I) GO TO 96

DO $1 I=1$, NDAIR

DO $1 \mathrm{~N}=1$, IH1

IF (N.LE.2) GO TO 93

$R X=X D U M(N+1, I)-X D U H(N, I)$ 


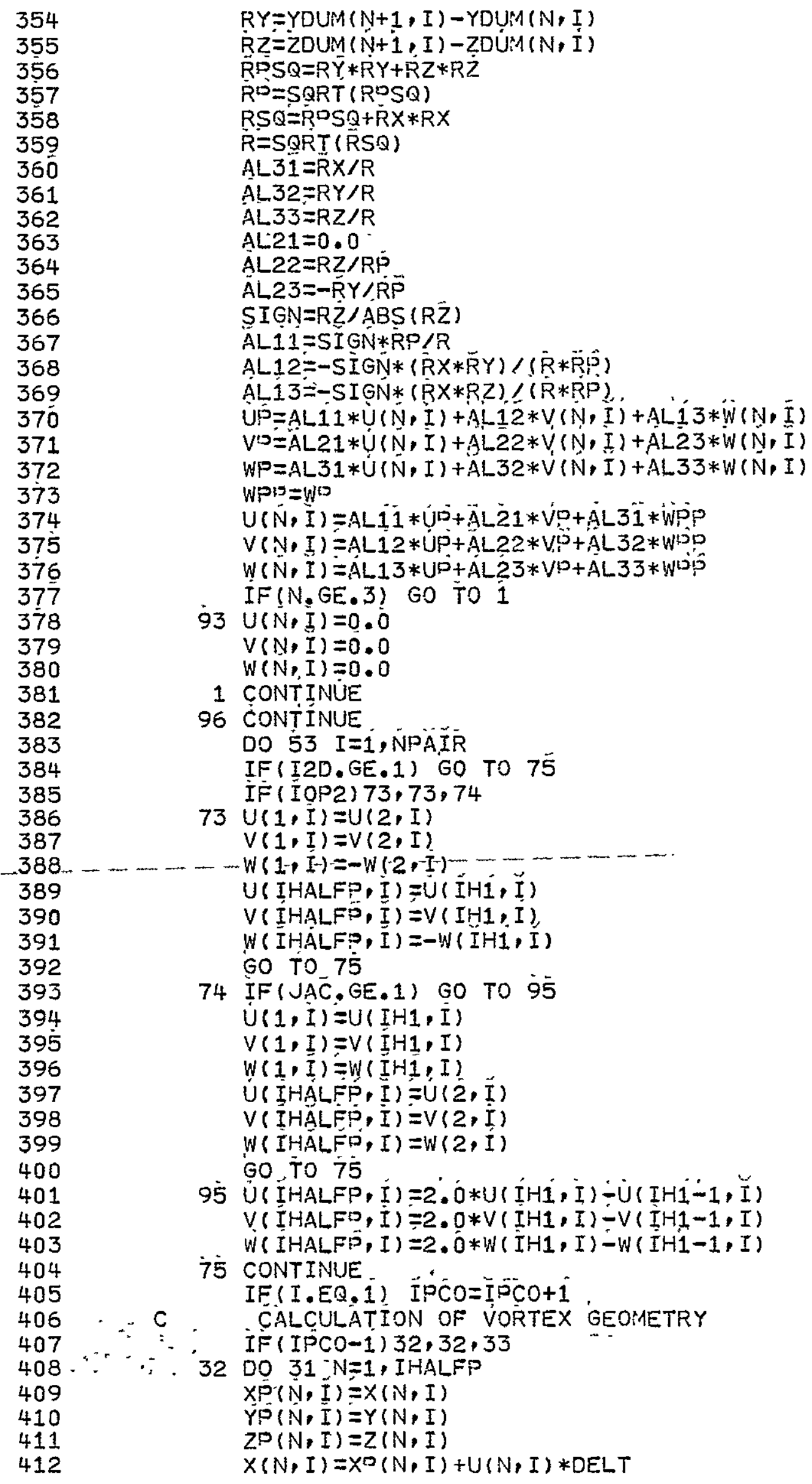

$R Y=Y D U M(N+1, I)-Y D U R(N, I)$

$\dot{R} Z=Z D U M(N+1, I)-Z D U N A(N, \dot{I})$

RिPSQR $=R+R Y+R Z * R \dot{Z}$

$R^{D}=S Q R T(R D S Q)$

$R S Q=R P S Q+R X * R X$

$R=S Q R T$ (RSQ)

$A L 31=R X / R$

AL $32=R Y / R$

$A L 33=R Z / R$

AL $21=0.0$

ÄL22 $=R Z / R P$

$A L 23=-R Y / R P$

$S I G N=R \hat{Z} / A B S(R \bar{Z})$

AL11 $=S I G N * R P / R$

AL $12=-S I G N *(\bar{R} X * \bar{R} Y) /(\bar{R} * \ddot{R}(\hat{S})$

$A L 13=-S I G N *(R X * R Z) /(R * R P)$,

UP $=A L 11 * U(N, I)+A L 12 * V(N, I)+A L I 3 * \dot{W}(N, \bar{I})$

$V^{\prime}=A L 21 * U(N, I)+A L 22 * V(N, I)+A L 23 * W(N, I)$

$W P=A L 31 * U(\hat{N}, I)+A L 32 * V(\hat{N}, I)+A L 33 * W(\bar{N}, I)$

$W P I=W[D$

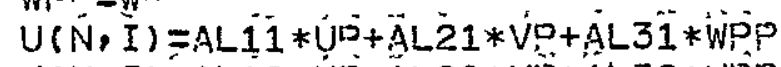

$V(N, I)=A L 12 * U P+A L 22 * V O A L 32 * W D$

$W(N, I)=A L 13 * U P+A L 23 * V P+A L 33 * W D$

$93 U(N, I)=0.010$ TO 1

$V(N, I)=0.0$

$W(N, I)=0.0$

1 CONTINUE

96 CONTÍNUE

DO 53 I=1, NPAIL

IF (I2D.GE.1) GO TO 75

İ ( IOPQ $73,73,74$

$73 U(1, I)=U(2, I)$

$V(1, I)=V(2, I)$

- - W $W(1, I)=-W(2,-T)$

$W(I, I)=-W(2,-I)$
$U(I H A L F P, I)=U(I H I, I)$

$V($ IHALFP, I $)=V(I H 1, I)$,

$W(I H A L F, I)=-W(I H I, I)$

GO TO 75

74 IF (JAC, GE.1) GO TO 95

$U(1, I)=U(I H 1, I)$

$V(1, I)=V(I, H I, I)$

$W(1, I)=W(I H I, I)$

$U(I H A L F P, I)=U(2, I)$

$V(I H A L F \bar{P}, I)=V(2, I)$

$W(I H A L F P, I)=W(2, I)$

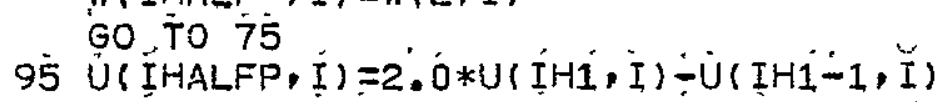

$V(I H A L F O, I)=2.0 * V(I H 1, I)-V(I H I-1, I)$

.W $W($ IHALFP, I $)=2.0 * W(I H 1, I)-W(I H 1-1, I)$

75 CONTINUE

CONTINUE

$\because C$ C CALCULATION OF VORTEX GEOMETRY

IF (IPCO-1) $32,32,33$

DO $31 \mathrm{~N}=1$. I HALFP

$X P(N, I)=X(N, I)$

$Y P(N, I)=Y(N, I)$

$Z P(N, I)=Z(N, I)$

$X(N, I)=X O(N, I)+U(N, I) * D E L T$ 
413

414

415

416

417

418

419

420

421

422

423

424

425

426

427

428

429

430

431

432

433

434

435

436

437.

438

439

440

441

442

443

444

445

446

447

448

449

450

451

452

453

454

455

456

457

458

459

460

461

462

463

464

465

466

467

468

469

470

471
$Y(\bar{N}, I)=Y \bar{P}(\bar{N}, \dot{I})+\dot{Y}(\bar{N}, \ddot{I}) * D E L T$

$31 Z(N, I)=Z P(N, I)+W(N, I) * D E L T$

GD TO 35

$33 \mathrm{DO} 34 \mathrm{~N}=1$, İHÄLP?

$X(N, I)=0.5 *(X P(\bar{N}, I)+X(N, I)+U(N, I) * D E L T)$

$Y(\bar{N}, I)=0.5 *(Y D(N, I)+Y(N, I)+V(N, I) * D E L, T)$

$34 Z(N, I)=0.5 *(Z, P(N, I)+Z(N, I)+W(N, I) * D E L T)$

IF $(J A C \cdot L E, O)$ GO TO 4

IF (IAC.GE.1), GO TO 38

DO 14 N $N=1$, IHALFP

NDE $=I H A L F P-N+1$

IF (INARCH.GE.I) NDE $=N$

IF (NDE.LE.2) GO TO 4

$I D P=1$

IF (IMARCH.GE.1) IDP $=-1$

$X(N D E+I D P, I)=X(N D E, I)$

$Y(N D E+I D P, I)=Y(N D E, I)$

$14 Z(N D E+I D D, I)=Z(N D E, I)$

4 CONTINUE

IF (JAC.LE. Ó) GO TO 38

- DO $399^{\circ} N=1,3$

$X(N, I)=X D(N, I)$

$Y(N, I)=Y \bar{N}(\bar{N}, \bar{I})$

$39 Z(N, I)=20(N, I)$

IF (IMARCH.LE.0), GO TO 38

$X(I H A L F P, I)=X P$ (IHALFP, I)

$Y(I H A L F P, I)=Y P(I H A L F P, I)$

$Z(I H A L F P, I)=Z^{D}$ (IHALFP, I)

38 CONTINUE

IF (IAC.LE, 0 ), Gंo to 35

IF(IMARCH。GE.1), GO TO 35

$X X_{N}(K, I ; U)=X(1, I)$

$Y I N(K, I, J)=Y(1, I)$.

$Z I N(K, I, U)=Z I N(K-I, I, J)+D E L T$

35 CONTINUE

IF (I2D.GE. i) GO TO 53

DELTZ $=(Z(I H 1, I)+Z(\dot{2}, I)) / 2.0$

$\operatorname{IF}(I 0.2 . L T .1)$ DELTZ $=0.0$

IF (JAC.LE. 0) $Z(1, I)=Z(1, I)-D E L T Z$

DO $47^{\circ} \mathrm{N}=2$, I HALFP

$\mathrm{N} M=\mathrm{N}-1$

IF $(J A C \cdot L E \cdot O), Z(N, I)=Z(N, I)-D E L T Z$

XOUMA $(N, I)=0.50 *(X(N, I)+X(N * 1, I))$

$\dot{Y D U M}(\tilde{N}, I)=0.50 *(Y)(N, I)+Y(N), I))$

$47 Z D U M(N, I)=0.50 *(Z(N, I)+Z(N M, I))$

DO $49 \mathrm{~N}=2$, IH1

$N P=N+1$

$C X(N, \bar{I})=C \operatorname{IRC}(I) *(X D U N(N, I)-X D U M A(N P, I))$

$C Y(N, I)=C I R C(I) *(Y D U N A(N, I)-Y D U M(N P, I))$

$49 \mathrm{CZ}(N, I)=C \operatorname{IRC}(I) *(Z D U M(N, I)-Z D U M(N D, I))$

53 CONTINUE

IF (IDCO.LE. I) GO TO $30^{\circ}$

TIME $=$ TIMUE+DELT

50 CONTINUE.

IF (I.AC.GE.1) GO TO 92

DO $85 I=1$,NPAIR

85 WRITE $(I W, 119) I, N, X(N, \bar{I}), Y(N, I), Z(\bar{N}, I), R C O M(\bar{N}, \bar{I})$
ORIGINAI' PAGE IS

OF POOR QUALITY 


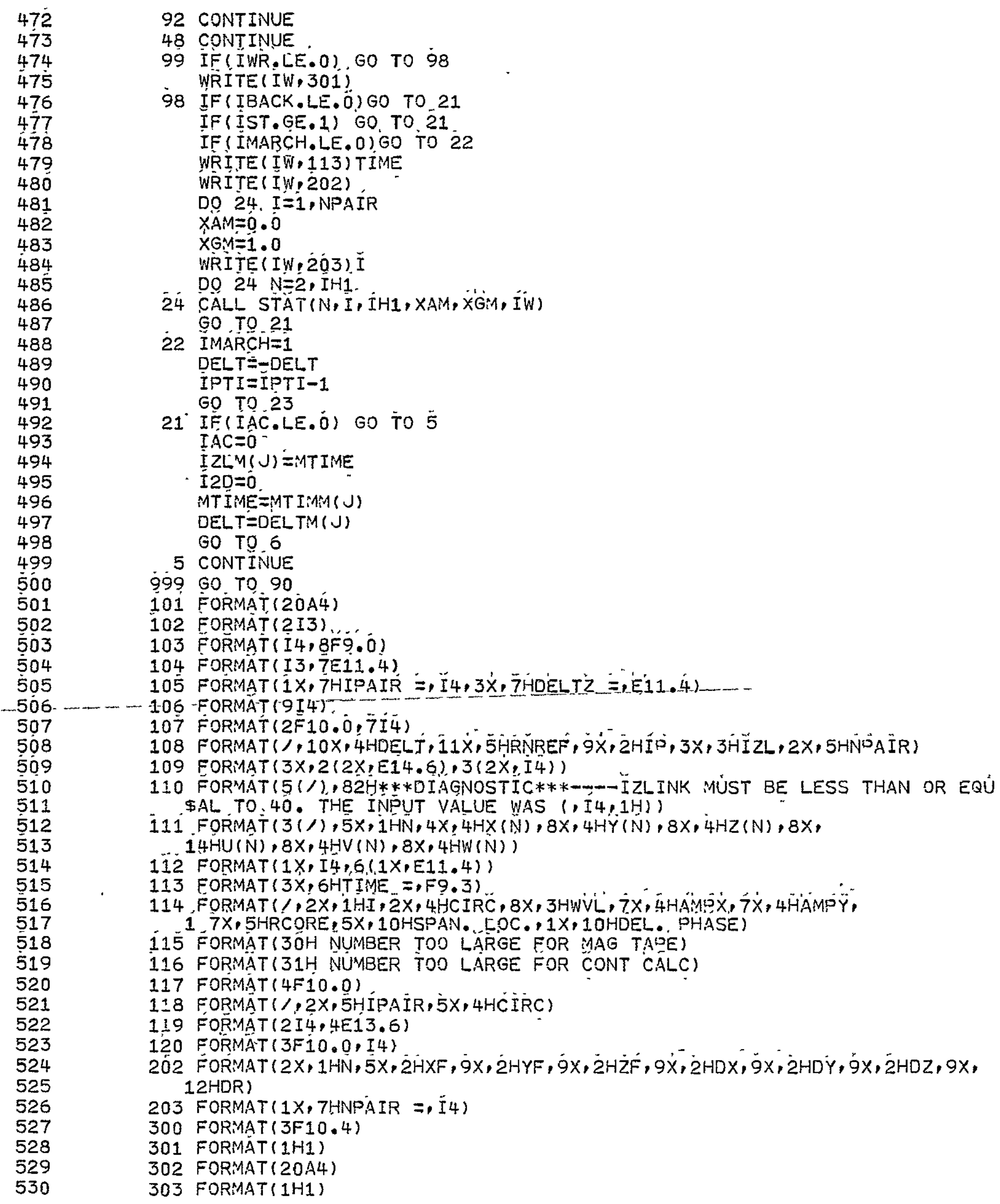

92 CONTINUE

48 CONTINUE.

99 IF (IWR.LE.0), GO TO 98 WRITE (IW, 301)

98 IF (IBACK.LE. O) GO TO 21

IF (IST.GE.1) GO. TO 21

IF (IMABCH.LE.0) GO TO 22

WRITE (IW, 113) TIME

WRITE (IW, 202)

DO 24. I $=1$, NPAIR

XAM $=0.0$

$X G M=1.0$

WRITE ( IW, 203 ) I

DO $24 \mathrm{~N}=2, \mathrm{IH} 1$.

24 CALL STAT $(N, \bar{I}, \dot{I} H 1, X A M, \ddot{X G}: 1, I W W)$

GO TO 21

22 IMARCH $=1$

DELT $=-D E L T$

IPTI $=I P T I-1$

GO TO. 23

$21^{\circ}$ IF (IAC.LE.Ó) GO TO 5

$I A C=0^{-}$

IZLM(J) =NTIME

- $I 2 D=0$.

MTIMEFMTIINM(U)

DELT $=D E L T M(J)$

GO TO 6

5 CONTINUE

999 GO TO 90

101 FORMAT'(20A4)

102 FORMAT (2I3)

103 FORMAT (I $4,8 F 9.0$ )

104 FORMAT (I $3,7 E 11.4)$

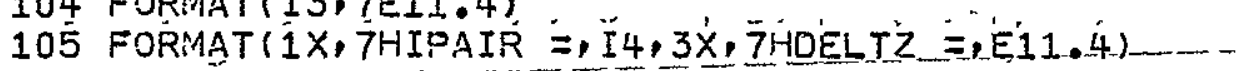

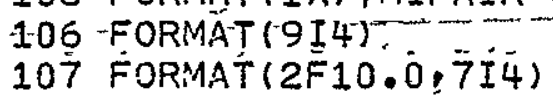

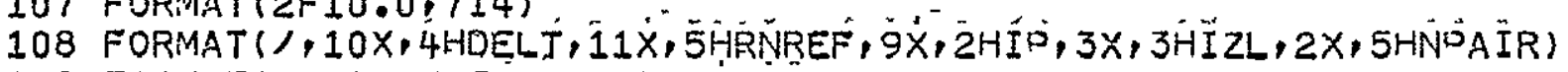

109 EORMAT $(3 X, 2(2 X, E 14,6), 3(2 X, I \overline{1}))$

110 FORMAT $(5(1), 82$ H***DIAGNOSTIC***---DIZINK MUST BE LESS THAN OR EQU \$AL TO 40 . THE INPUT VALUE WAS $($ I I $4,1 H)$

111 FORMAT $(3(), 5 X, 1 H N, 4 X, 4 H X(N), 8 X, 4 H Y(N), 8 X, 4 H Z(N), 8 X$,

$14 H U(N), 8 X, 4 H V(N), 8 X, 4 H W(N))$

112 FORMAT $(1 \times$, I $4,6,(1 \times, E 11.4))$

113 EORMAT (3X, 6HTIME $=, F 9.3)$.

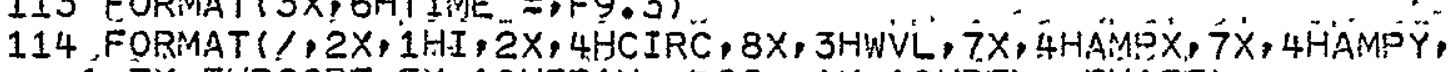

$1,7 X, 5$ HRCORE, $5 X, 10 H$ SPAN. EOC., $1 X, 10$ HDEL. PHASE)

115 FORMAT ( 30 H NUMBER TOO LARGE EOR MAAG TADE)

116 FORMAT (31H NUMBER TOO LARGE FOR CONT CÁLC)

117 FORMAT $(4 F 10.0)$

$1 \pm 8$ FORMAT ( /,2X,5HIPAIR, 5X, 4HCIRC)

119 FORMAT $(2 I 4,4 E 13.6)$

120 FORMAT (3F10,0,I4)

202 FORMAT $(2 X, 1 H N, \overline{5} X, \dot{2} H X F, \dot{9} X, \dot{2} H Y F, \dot{9} X, \dot{2} H \dot{Z} F, 9 \dot{x}, \dot{2} H D X, \dot{9} X, \dot{2} H D Y, \dot{9} X, \dot{2} H D Z, 9 x$, 12HDR)

203 FORMAT $(1 X, 7$ HNPAIR $=$, I 4$)$

300 FOR:MAT (3F10.4)

301 FORMAT (1H1)

302 FORMAT(20A4)

303 FOR:AT (1H1) 
304 FORMAT(2X, $44 \times C G=, E 11,4,6 H \quad Y C G=, E 11.4,9 H \quad$ VDRIFT $=, E 11.4$, 305 FORMAT $(2 X, 5 H X M A C=, F 10.4)$

998 CONTINUE

STOD

END 


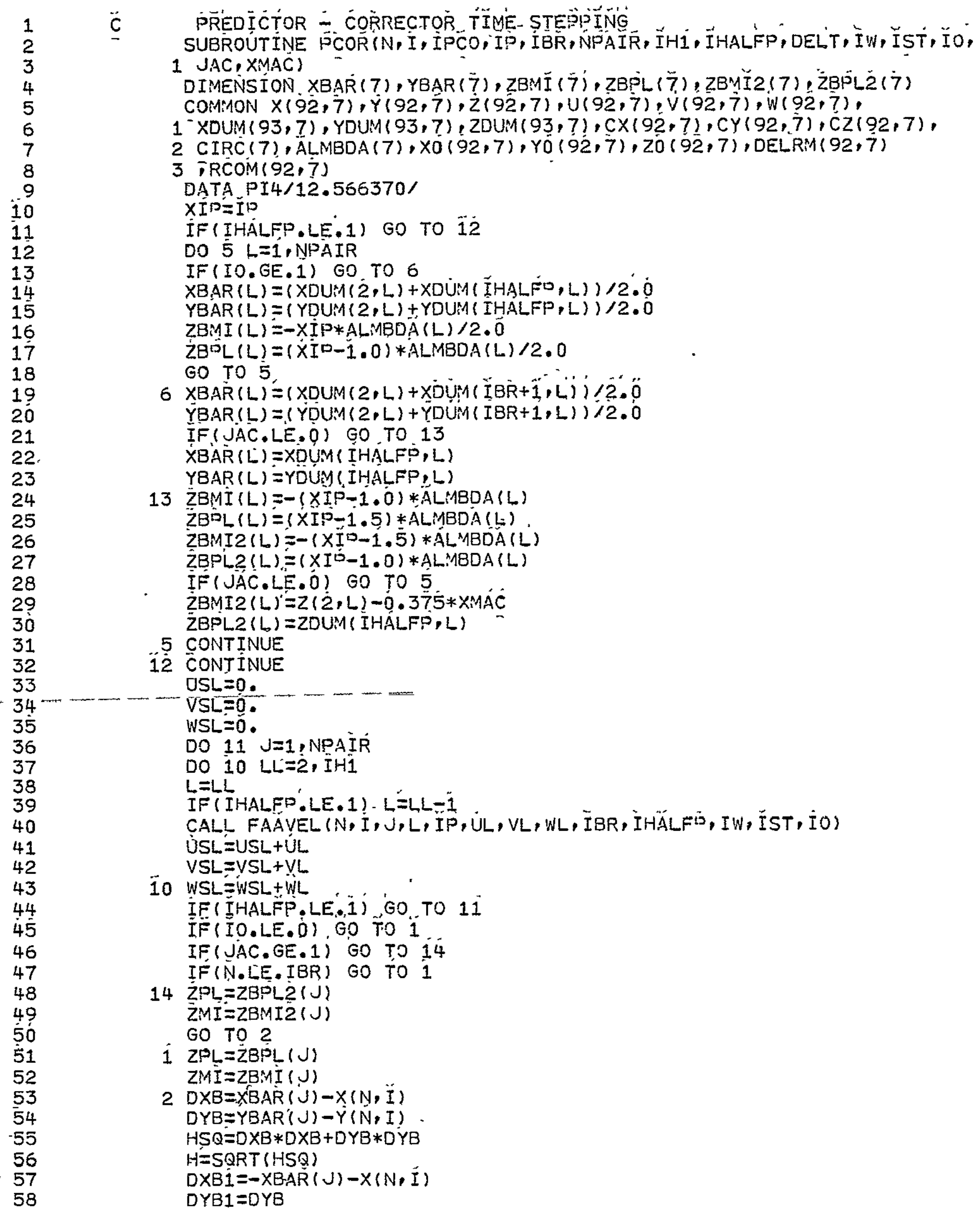




\begin{tabular}{|c|c|c|}
\hline $\begin{array}{l}59 \\
60 \\
61 \\
62 \\
63 \\
64 \\
65 \\
66 \\
67 \\
68 \\
69 \\
70 \\
71 \\
72 \\
73 \\
74 \\
75 \\
76 \\
77 \\
78 \\
79 \\
80 \\
81 \\
82 \\
83 \\
84 \\
85 \\
86 \\
87 \\
88 \\
89 \\
90 \\
91 \\
92 \\
93 \\
94 \\
95 \\
96\end{array}$ & $\begin{array}{l}20 \\
21 \\
11\end{array}$ & 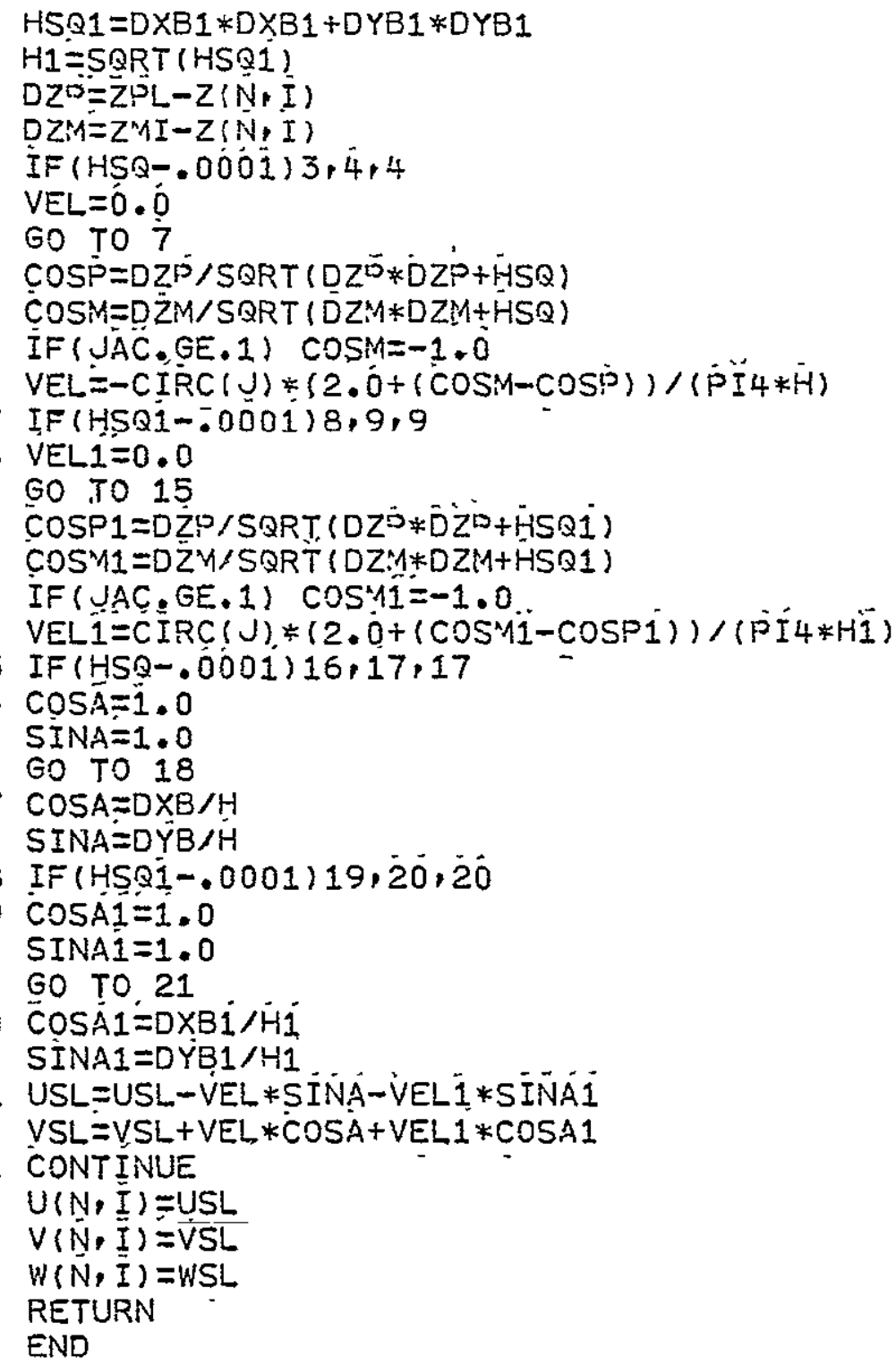 \\
\hline
\end{tabular}




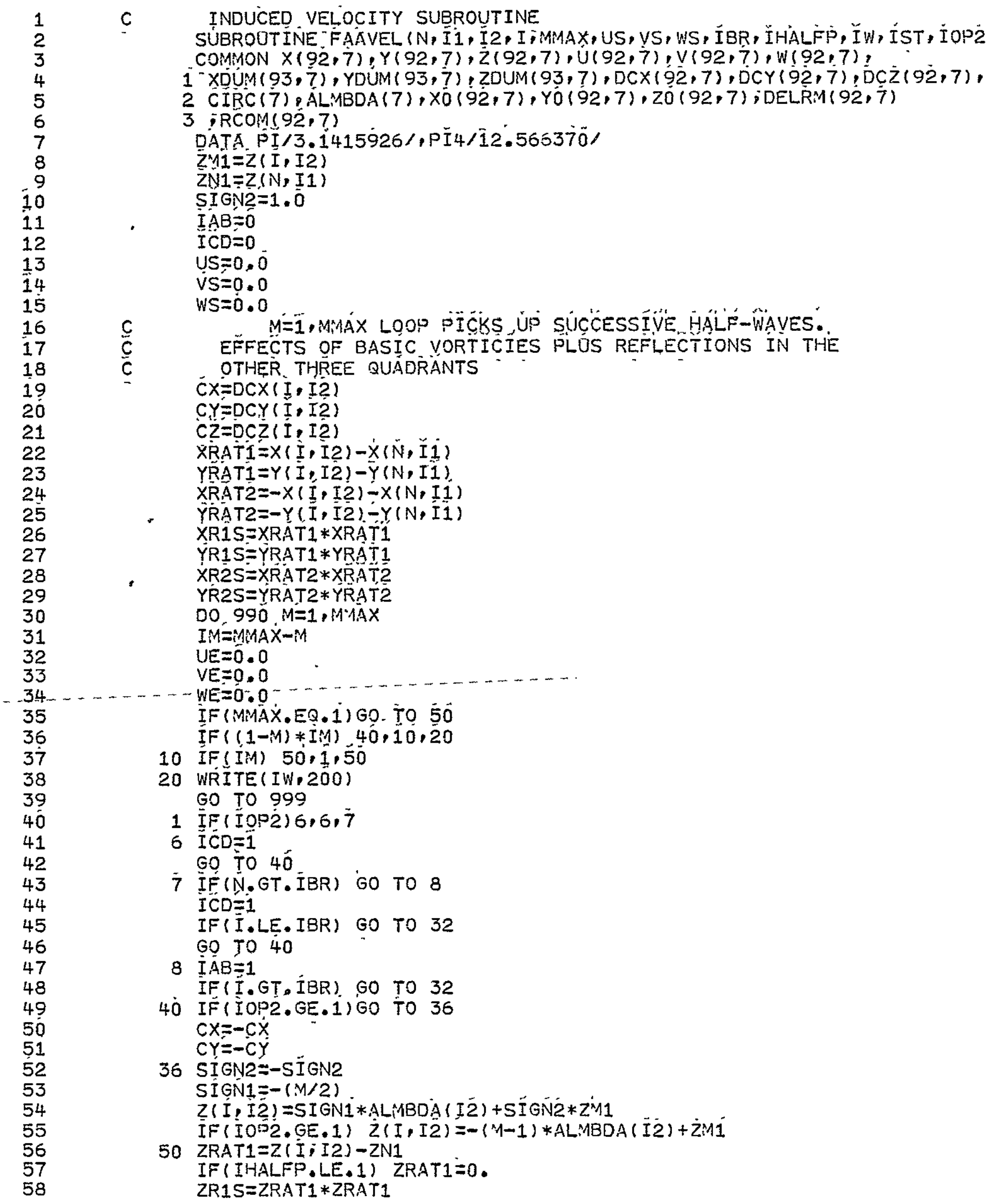
COMMON X $(92,7), Y(92,7), Z(92,7), U(92,7), V(92,7), W(92,7)$,

1-XDUM $(93,7), Y D U M(93,7), Z D U M(93,7), D C X(92,7), D C Y(92,7), D C Z(92,7)$,

2 CIRC (7), ALABBDA (7), XO $(92,7), Y O(92,7), Z O(92,7)$; DELRM $(92,7)$ 3 :RCOM(92,7) DATA PIJ3.1415926/,PI $4 / 12.5653700 /$ $Z M 1=Z(I, I 2)$

$I A B=0$

$I C D=0$

US $=0.0$

$V S=0.0$

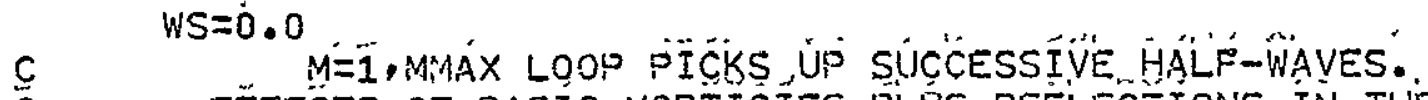

EFFECTS OF BASIC VORTICIES FLÓS REFLECTIONS IN THE

C OTHER THREE QUADRANTS

$\bar{C} X=D C X(1,12)$

$C Y=D C Y Y(I, I 2)$

$C Z=D C Z(I, I 2)$

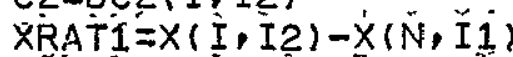

$Y R A T I=Y(I, I 2)-\bar{Y}(N, I 1)$

$X R A T 2=-X(I, I 2)-X(N, I I)$

- YRAT2 $=-Y(I, I 2)-Y(N, I 1)$

XR1S $X X R A T 1 * X R A T 1$

YR1S=YRAT1*YRAT1

$X R 2 S=X R A T 2 * X R A T 2$

YR2S $=$ YRAT2* YRAT2

DO, $990, M=1 \cdot M M A X$

IM $=$ IMMAX-M

$\mathrm{UE}=0.0$

$V E=0.0$

- WE $=0.0$

IF (MMÁX.EQ.i)GO TO 50

$I F((1-M) * I M) \quad 40,10,20$

$10 \operatorname{IF}(I M) 50,1,50$

20 WRITE (IW, 200)

GO TO 999

1 IF (IOP之) $6,6,7$

6 ICD $=1$

GO TO 40

7 IF (N.GT.IBR) GO TO 8

ICD $=1$

IF (I.LE.IBR) GO TO 32

GO TO 40

$8 I A B=1$

IF (I.GT, IBR), GO TO 32

40 IF (IOP2.GE.1)GO TO 36

$C X=-C X$

$C Y=-C Y$

36 SIGN2 $=-S I G N 2$

SIGN1 $=-(M / 2)$

$Z(I, I 2)=S I G N 1 * A L M B D A(I \check{2})+S I G N 2 * Z^{*} 1$

IF (IOD2. GE.1) $Z(I, I Z)=-(M-1) * A L M B D A(I 2)+Z M 1$

50 ZRAT1 $=Z(I ; I 2)-Z \mathrm{~N} 1$

IF (IHALFP.LE.1) ZRAT1 $=0$.

$Z R 1 S=Z R A T 1 * Z R A T 1$ 
59

60

61

62

63

64

65

66

67

68

69

70

71

72

73

74

75

76

77

78

79

80

81

82

83

84

85

86

87

88

89

90

91

92

93

94

95

96

97

98

99

100

101

102

103

104

105

106

107

108

109

110

111

112

113

114

115

116

117

IF (IAB,'GE. I) GO TO 2

RSQA $=X R 1 S+Y R 1 S+Z R I S$

IF (IOP2.LE.0) GO TO 37

$2 Z R A T 2=Z(I, I 2)+(2 * M-2) * A L M B D A(I 2)-Z \bar{N} \bar{I}$

IF (IHALFP. LE.1) ZRÄTZ=0.

GO TO 38

37 ZRAT2 $=-Z(I, I 2)-Z N 1$

38 ZR2S $=Z R A T 2 * Z R A T 2$

IF (I.AB.GE.1) GO TO 3

$R S Q B=X R 2 S+Y R 1 S+Z R 1 S$

IF (IHALFD.LE.1) GO TO 43

IF (ICD.GE.1) GO TO 5

$3 R S Q C=X R 2 S+Y R 1 S+Z R 2 S$

$R S Q D=X R 15+Y R 1 S+Z R 2 S$

43 IF (IAB.GE.1) GO TO $4 \ldots$

5 IF (RSQA-.0001)11,12,12

11 RECIPA $=0.0$

GO:TO 13

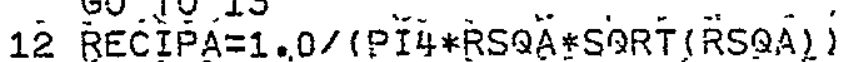

IF (IHALFP.LE.1) RECIPA $=1.0 /(2.0 * P I * R S Q A)$

13 IF (RSQB- .0001$) 14,15,15$

14 RECIPB $=0.0$

GO TO 16

15 RECIPB $=1.0 \%(P+4 * \bar{R} S Q B * S Q R T(\vec{R} S Q B))$

IF (IHALFP, LE. 1) RECIPB $=1.0 /(2.0 * D I * R S Q B)$

16 IF(ICD.GE. I) GO TO 22

IF(IHALFP.LE. I) GO TO 17

4 IF $(R S Q C-.0001) 17,18,18$

17 RECIPC $=0.0$

GO-TO:19.

I8 RECIDC=1.0/(PI $4 * R S Q C * S Q R T(R S Q C))$

19 IF (IHALFP.LE.1) GO TO 23

IF (RSQD $=.0001) 23.21,21$

23 RECIDD $=0.0$

$60^{-} \mathrm{TO} 22$

21 RECIPD $=1.0 /(P I 4 * R S Q D * S Q R T(R S Q D))$

22 IF (IAB.GE.1) GO TO 30

UE $=R E C I P A *(C Z * Y R A T I-C Y * Z R A \ddot{T} I)$

VE $=R E C I P A *(C X * Z R A T 1-C Z * X R A T 1)$

WE =RECIPA* (CY*XRAT1-CX*YRAT1).

UE $=U E F R E C I D B *(C Y * Z R A T 1-C Z * Y R A T 1)$

$V E=V E+R E C I D B *(C X * Z R A T 1+C Z * X R . A T 2)$

$W E=W E+R E C I P B *(-C Y * X \bar{R} A T 2-C X * Y R A T 1)$

IF (ICD.GE. I) $60^{\circ}$ TO 32

IF(IOP2. GE.1) GO TO 30 .

UE $=U E+R E C I P C *(-C \dot{Z} * Y R A T I-C Y * Z R A T T)$

$V E=V E+R E C I P C *(-C X * Z R A T 2+C Z * X R A T 2)$

WE $=W E+R E C I P C *(C Y * X R A T 2+C X * Y R A T 1)$

UE $=U E+R E C I P D *(C Z * Y R A T 1+C Y * Z R A T 2)$

$V E=V E+R E C T P D *(-C X * Z R A T Z=C Z * X R A T 1)$

WE $=W E+R E C I D D *(-C Y * X R A T 1+C X * Y R A T 1)$

GO TO 32

30 IF (M.EQ.1) GO TO 32

UE $=U E+R E C I P C *(-C Z * Y R A T 1+C Y * Z R A ́ T 2)$

$V E=V E+R E C I P C * 1+C X * Z R A T 2+C Z * X R A T 2)$

$W E=W E+R E C I P C *(-C Y * X R A T 2-C X * Y R A T 1)$

UE $=U E+R E C I P D *(C Z * Y R A T 1-C . Y * Z R A T 2)$

$V E=V E+R E C I P D *(+C X * Z R A T 2=C Z * X R . A T 1)$

$W E=W E+R E C I P D *(+C Y * X R A T 1-C X * Y R A T 1)$ 


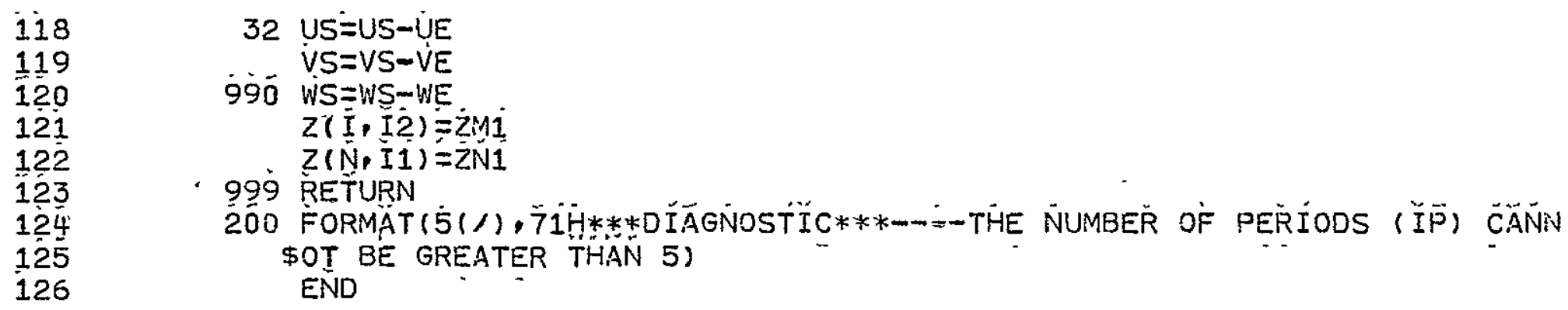

32 US=US-UE VS $=V S-V E$

990 WS=WS-WE

$Z(I, I 2)=Z M i$

$Z(N, I 1)=Z N 1$

- 999 RETURN

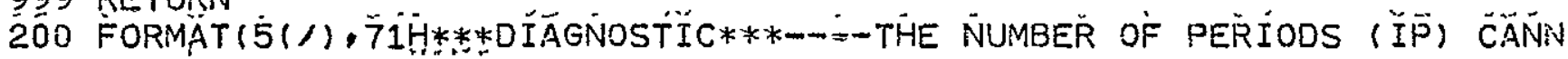
\$OT BE GREATER THAN 5) END 


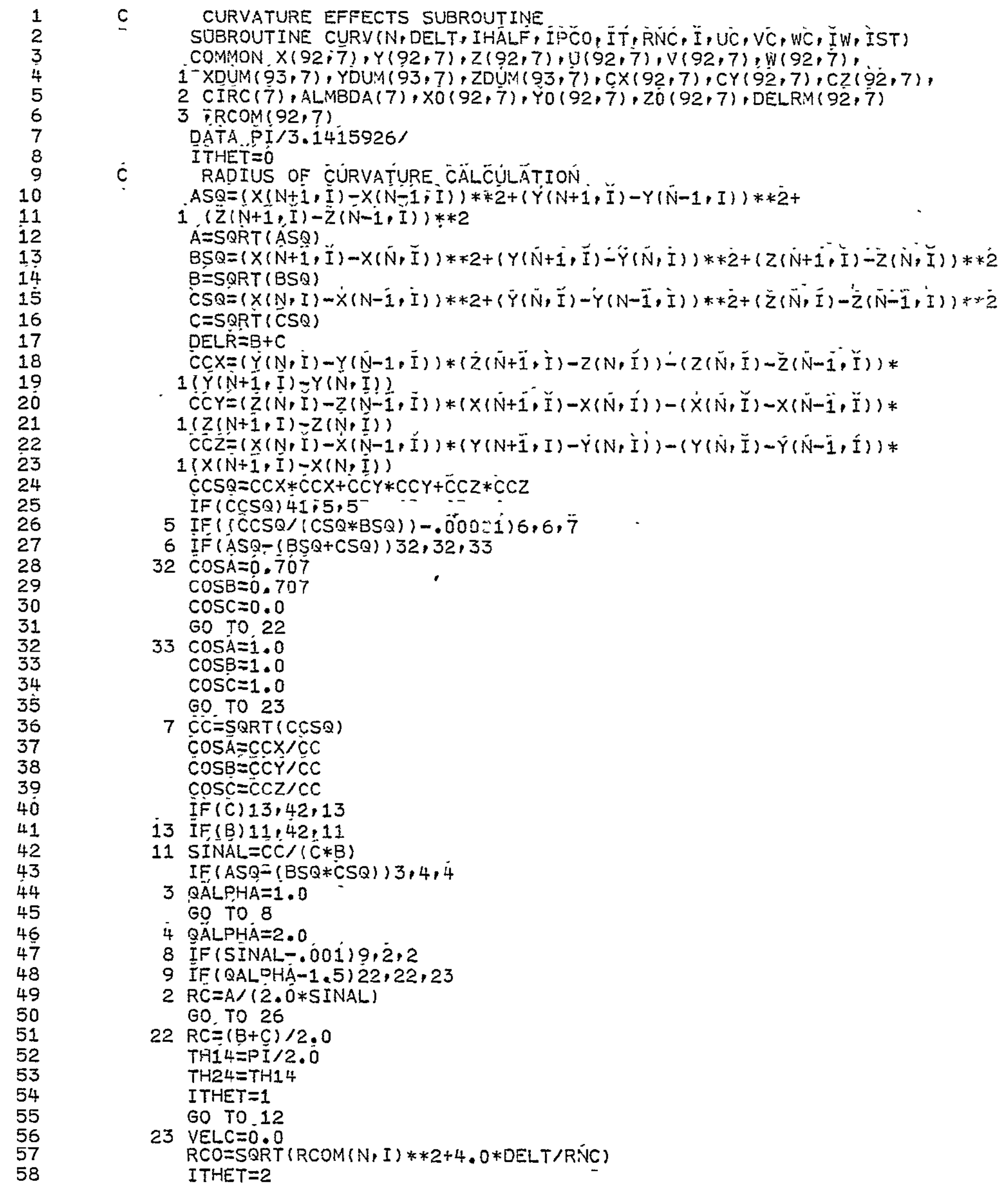




\section{GO TO 12}

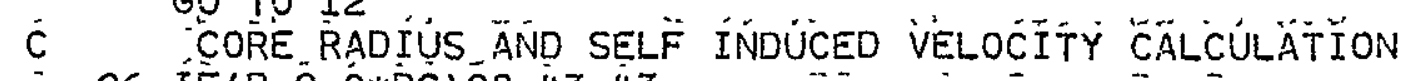

26 IF $(B-2.0 * R C) 28,43,43$

28 IF $(C-2,0 * R C) 27,43,43$

27 TH1 $4=0.25 * \bar{A} T A N(B /(S Q R T(\overline{4} \cdot \ddot{0} * R C * R C-B * B)))$ $T H 24=0.25 * A T A N(C)(S Q R T(4.0 * R C * R C-C * C)))$

12 IF (IT.GT.1) GO TO 34.

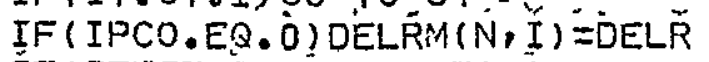

34 IF (ITHET.EQ.2) GO TO 10

$16 \mathrm{D} 2=R \operatorname{COM}(N, I) * * 2 *(D E L R-D E L R M(N) \dot{I})) / D E L R$ DRCO2 $=4.0 * D E L T / R N C-D 2$ RCO2=RCOM $(N, I) * * 2+D R C O 2$

IF (RCO2) 44, 44,29

$29 \dot{R} C O=S Q R T(R C O 2)$

IF (RC) $45,45,30$

30 DV $1=A L O G(8.0 * R C / R C O)-0.558$

IF (ITHET) $18.18: 19^{-}$

19 Dิ $2=0.0$

GO TO, 20

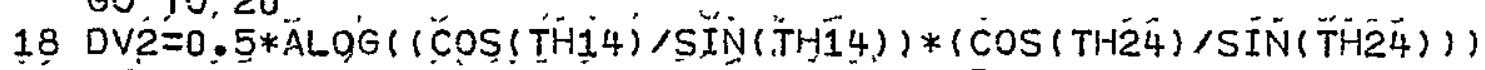

20 VELC $C I R C(I) *(D V I-D V Z) /(4.0 * D I * R C)$

10 UC $=V E L C * C O S A$

$V C=V E L C * \operatorname{COSB}$

$W C=V E L C * C O S \bar{C}$

IF (IPCO.GE. I) GO TO $\overrightarrow{1}$

$D E L R M(N, I)=D E L R$

$\operatorname{RCOM}(N, I)=\bar{R} C O$

GO TO 1

41 WRITE (IW, 100)

GO TO 46

42 WRITE (IW, 101)

GO TO 46

43 WRITE (IW, 102$)$

GO, TO 46

44 WRITE (IW, 103)

GO TO 46

45 WRITE $(I N, 104)$

46 IST $=1$

1 CONTINUE

RETURN

100 FORMAT (15H ABS, (CXB) .LT.Ö.).

101 FORMAT (23H ADJ. POINTS COINCIDENT)

102 FORMAT (24H RAD. OF CURV. TOO SMALL)

103 FORMAT (12H RCORE. LE.O.).

104 FORMAT (19H RAD. OF CURV.LE.Ö.) END 
1

2

C COMPÄISON OE GEOMETRYY ÄFTER BKWD. MÁRCH WITH INIT. GEOMETRY SUBROUTINE STAT (N, IFIH1, XAY + XGM, IW) COMANON $X(92,7), Y(92,7), Z(92,7), U(92,7), V(92,7), W(92,7)$, $1^{-}$XDUM $(93,7)$, YDUM $(93,7), Z D U M(93,7), C X(92,7), C Y(92,7), C Z(92,7)$, $2 \operatorname{CIRC}(7), \ddot{A L} M B D A(7), X 0(92,7), Y 0(92,7), Z 0(92,7), \operatorname{DELRM}(92,7)$ 3 iRCOM $(92,7)$ $D X=X(N, I)-X O(N, I)$ $D Y=\bar{Y}(\bar{N}, T)-\bar{Y} O(N, I)$ $D Z=Z ̈(N, I)-Z O N(N, I)$ $D R=S Q R T(D \dot{X} * D X+D \dot{Y} * D Y+D \dot{Z} * D \bar{Z})$ $X N \bar{P}=\mathrm{N}$

$X A M=X A M+D R$ $X G M=X G M * D R$ $\mathrm{NP}=\mathrm{N}-1$ WRITE $(6,200) N P, X(N, \ddot{I}), Y(N) \dot{I}), Z(N, I), D X, D Y, D Z, D R$ IF (N.LT.IHI) GO TO 25 $X A M E=X A M / X N P^{-}$ $X G M E=\left(X G^{M}\right) * *\left(\overline{1} . / X N N^{\prime}\right)$ 25 CONTINUE WRITE $(6,201) X A M E, X G$ :YE RETURN

200 FORMAT (I $3, \overline{7} E 11.4$ )

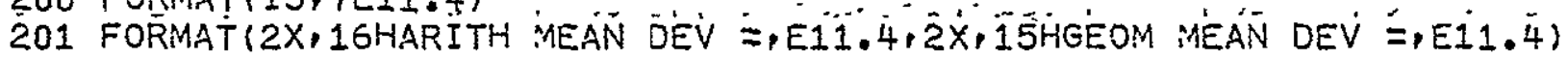
END

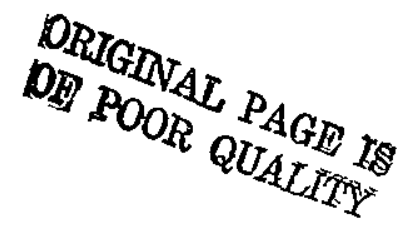




\section{FIGURES}

PRECEDING PAGE BLANK NOT FILMED 


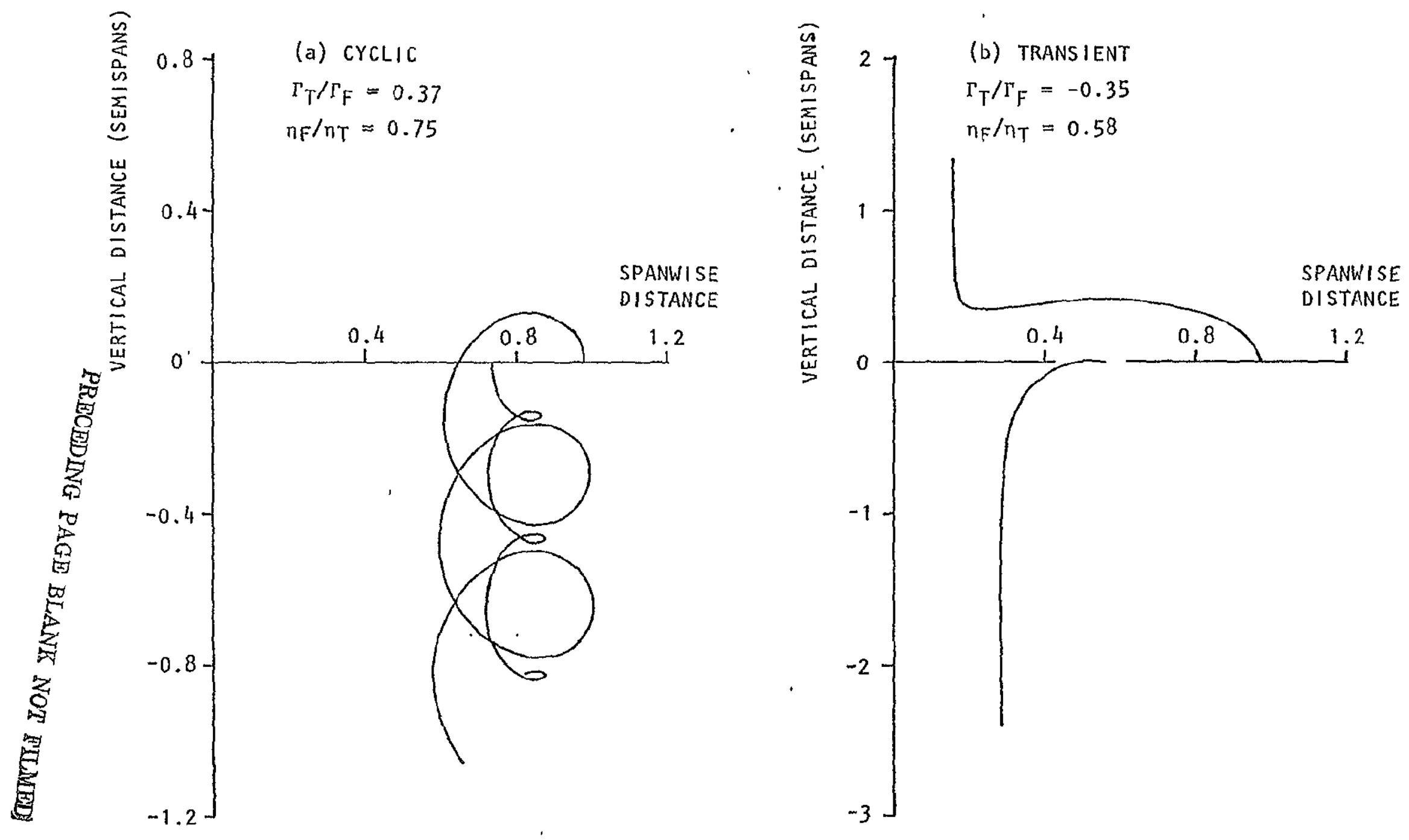

NOTE: DISTANCES NORMALIZED ON WING SEMI-SPAN 


\section{DEFINITION}

LOOPING OR CONVERGENCE OCCURS AT THE POINT WHERE THE LOCAL VERTICAL VELOCITY $=10$ TIMES THE DRIFT VELOCITY OF THE SYSTEM

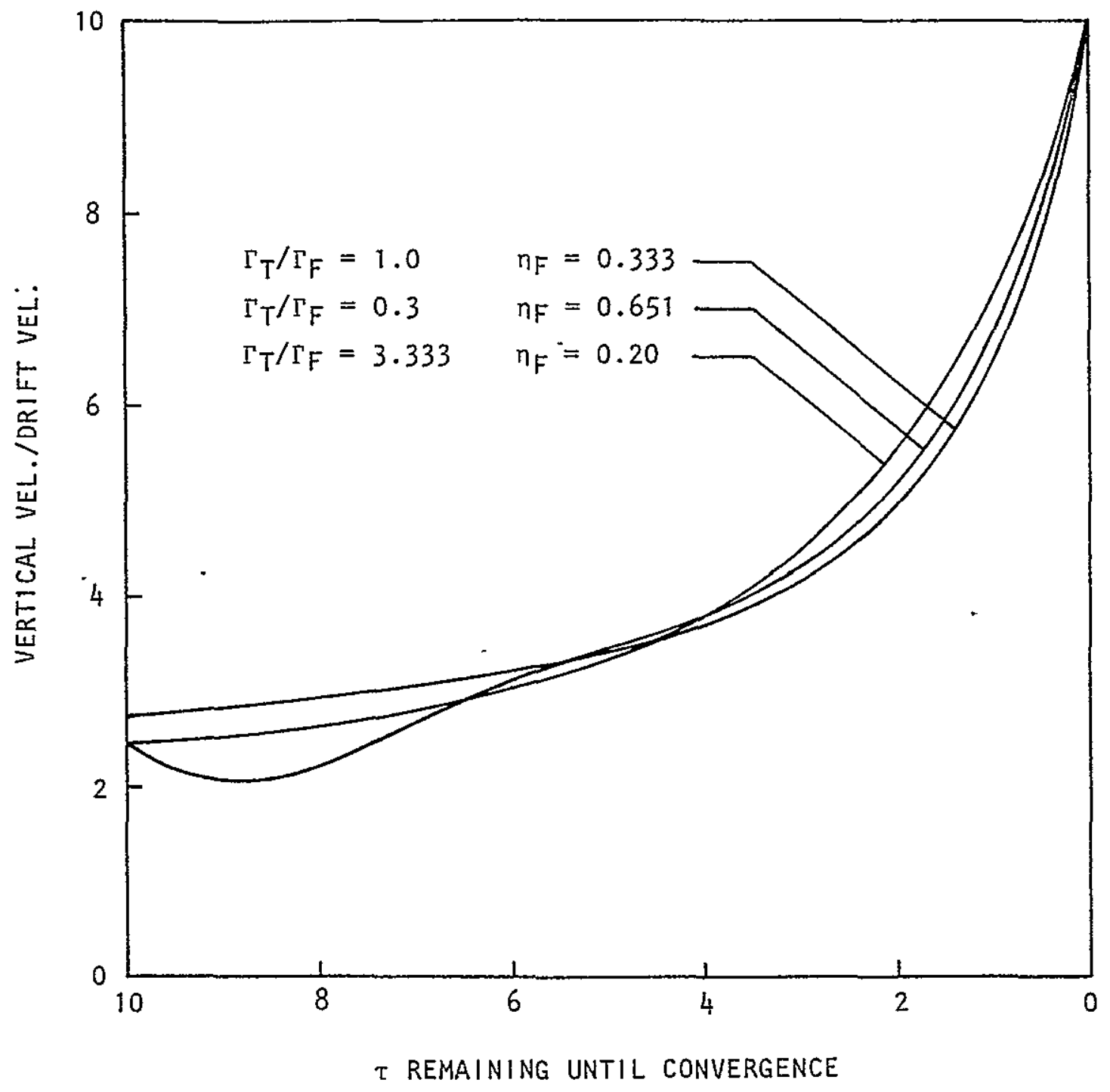

Figure 2.2 Definition of the Onset of Convergence 


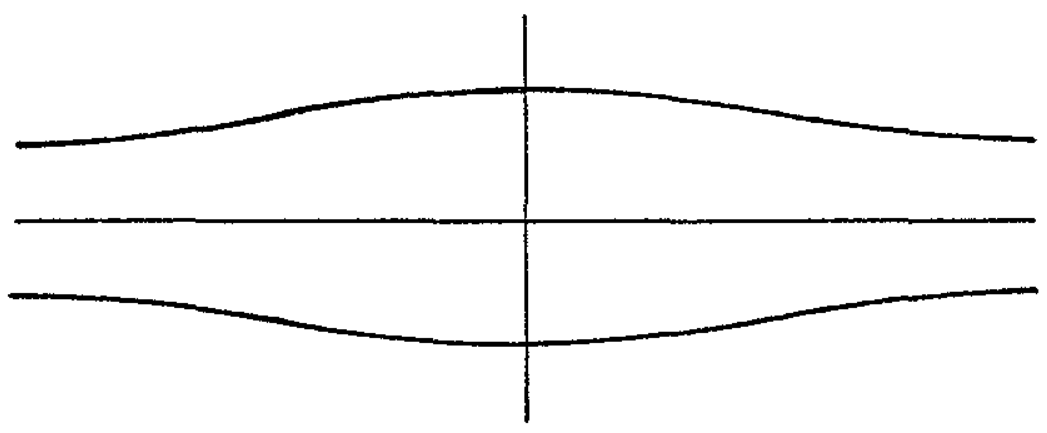

(a) INITIAL CONDITIONS

$$
\begin{aligned}
& \Gamma=0.127 U_{\infty} b \\
& \lambda=5.0 b \\
& a_{x}=a_{y}=0.125 b
\end{aligned}
$$

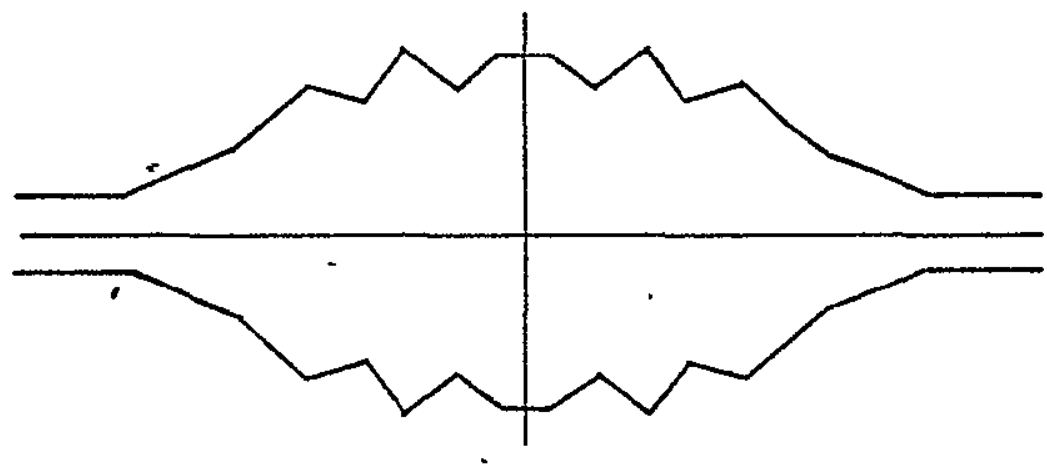

(b) CALCULATION FROM REF. 2 10 POINTS/HALF WAVE CORE UNSPECIFIED TIME $=5.33 \mathrm{~b}^{2} / \Gamma$

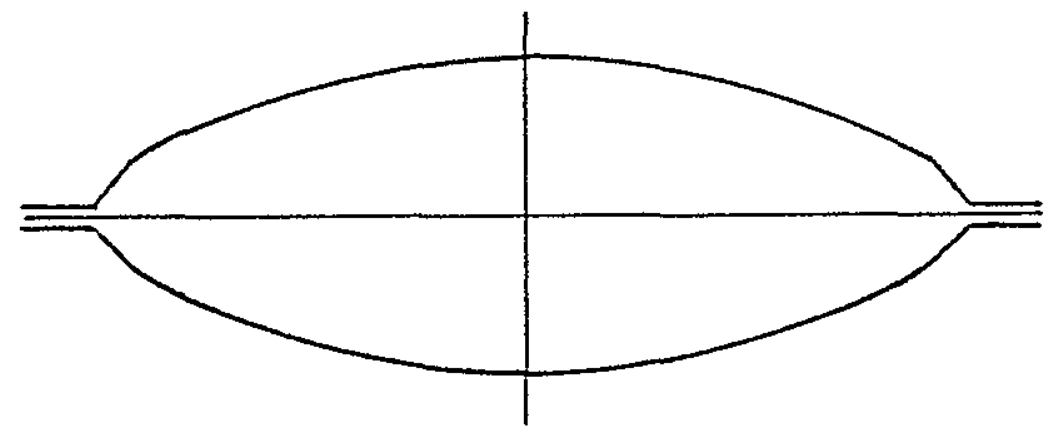

(c) PRESENT CALCULATION 10 POINTS/HALF WAVE CORE RADIUS $=.098 \mathrm{~b}$ TIME $=5.33 \mathrm{~b}^{2} / \mathrm{T}$

Figure 3.1 Previous and Present Calculations of the Distortion of a Vortex Pair 


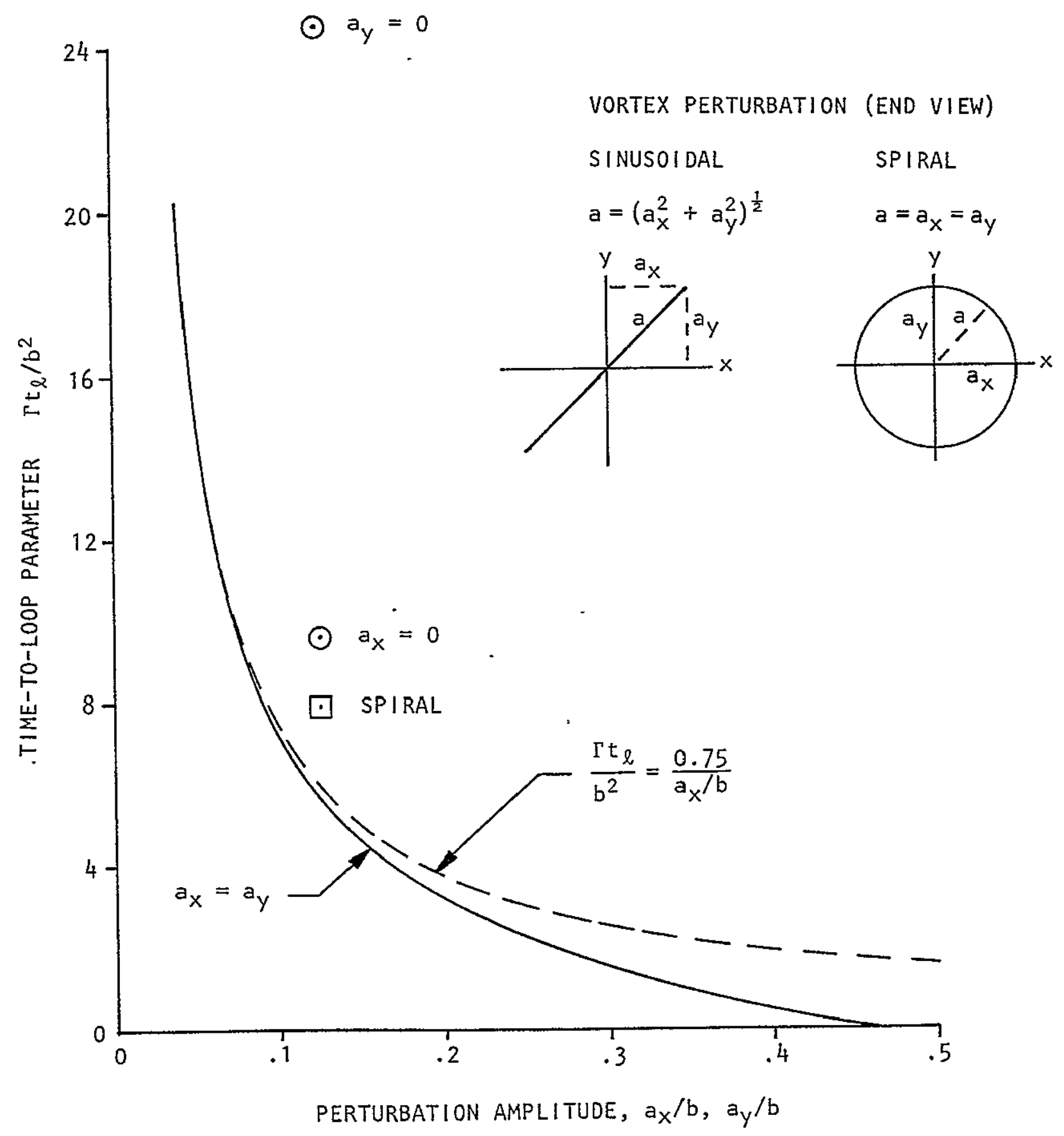

Figure 3.2 Dependence of Time-to-Loop upon Initial Perturbation Amplitude, $\lambda=5.0, \delta / b=.098$ 


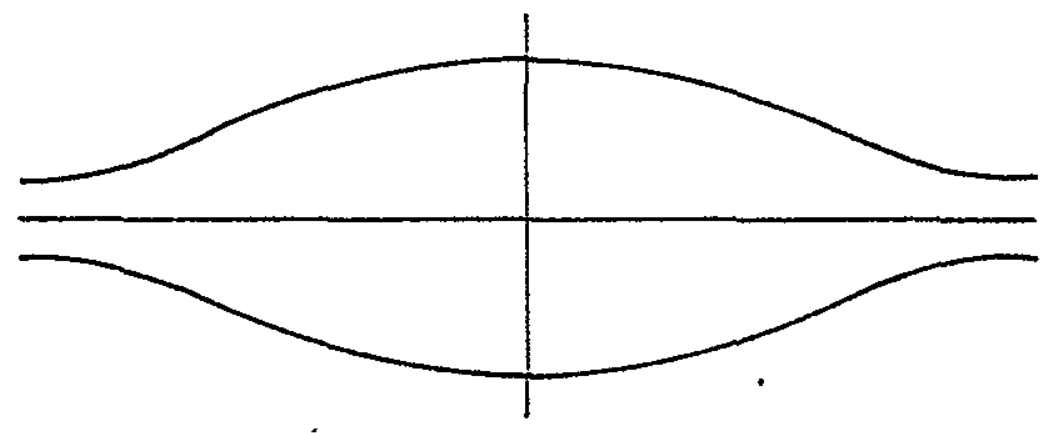

(a) NO CURVATURE EFFECTS

$\Gamma=0.127 \mathrm{U} \mathrm{b}$

$\lambda=5.0 \mathrm{~b}$

$a_{x}=a_{y}=0.125 b$

CORE UNSPECIFIED

TIME $=5.33 \mathrm{~b}^{2} / \mathrm{T}$
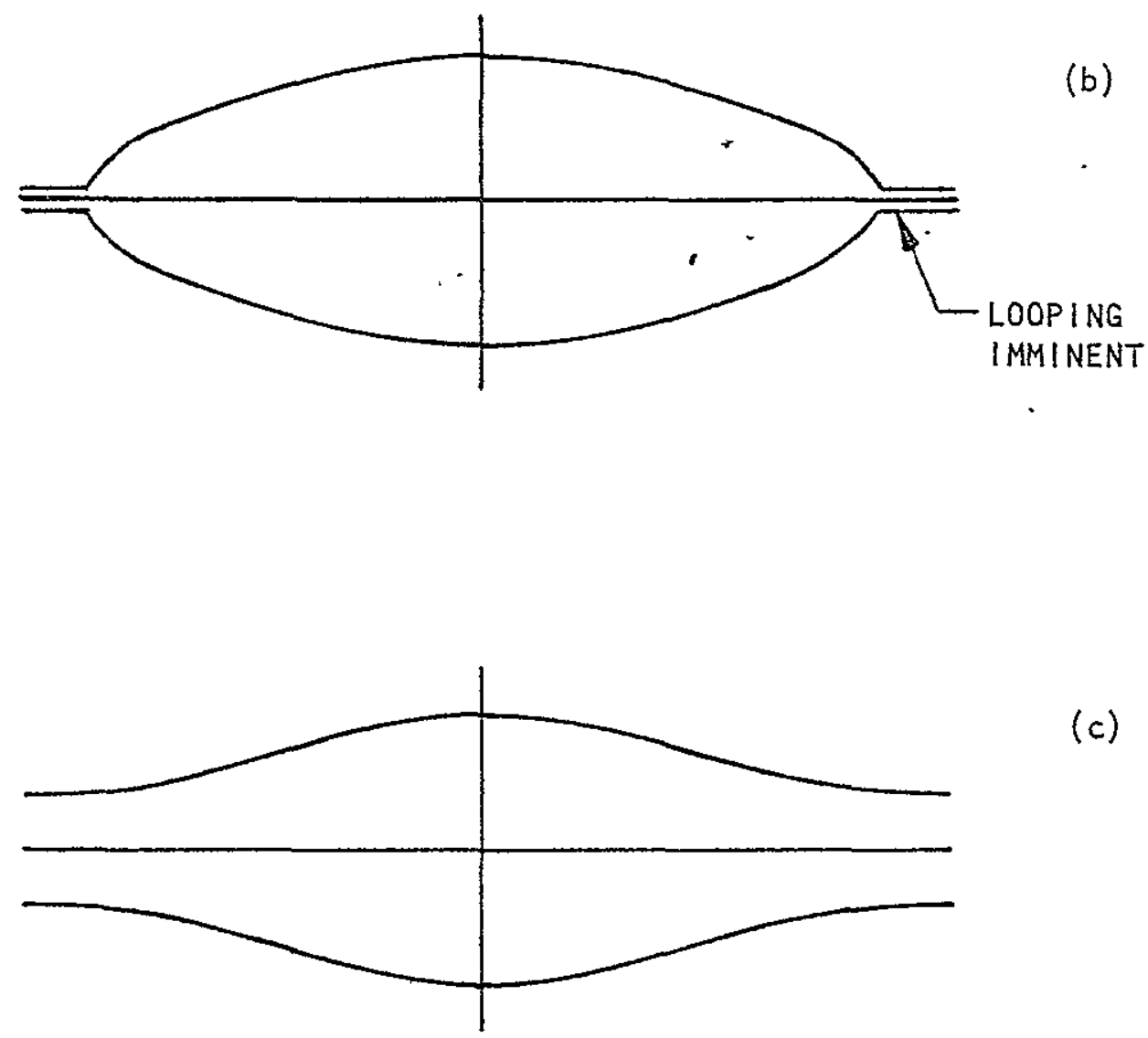

(c) CORE RADIUS $=.382 \mathrm{~b}$

TIME $=5.33 \mathrm{~b}^{2} / \mathrm{T}$

Figure 3.3 Effect of Vortex Core Radius on the Distortion of a Vortex Pair 


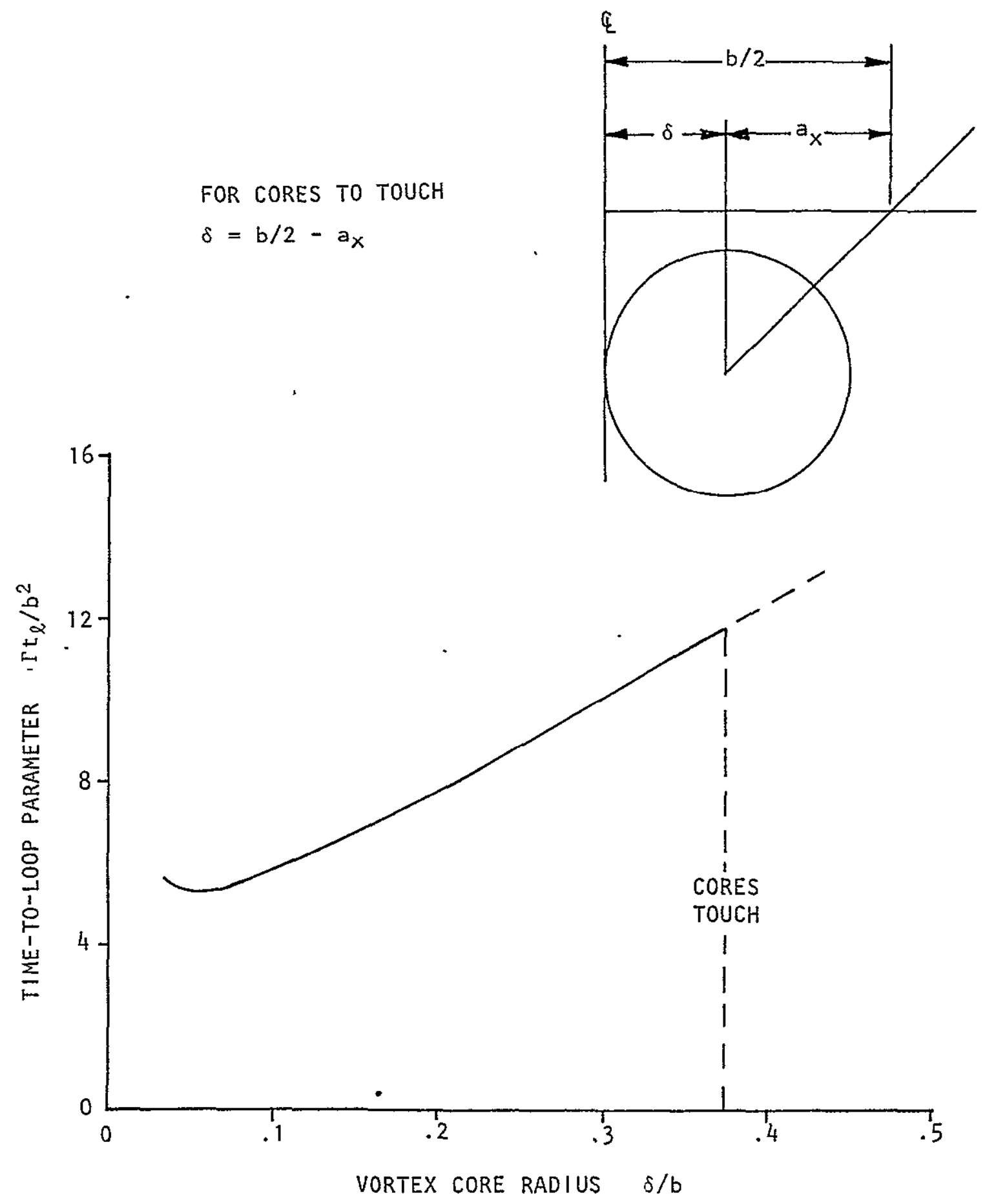

Figure 3.4 Dependence of Time-to-Loop Upon Vortex Core Radius, $a_{x} / b=a_{y} / b=0.125, \lambda=5.0$ 


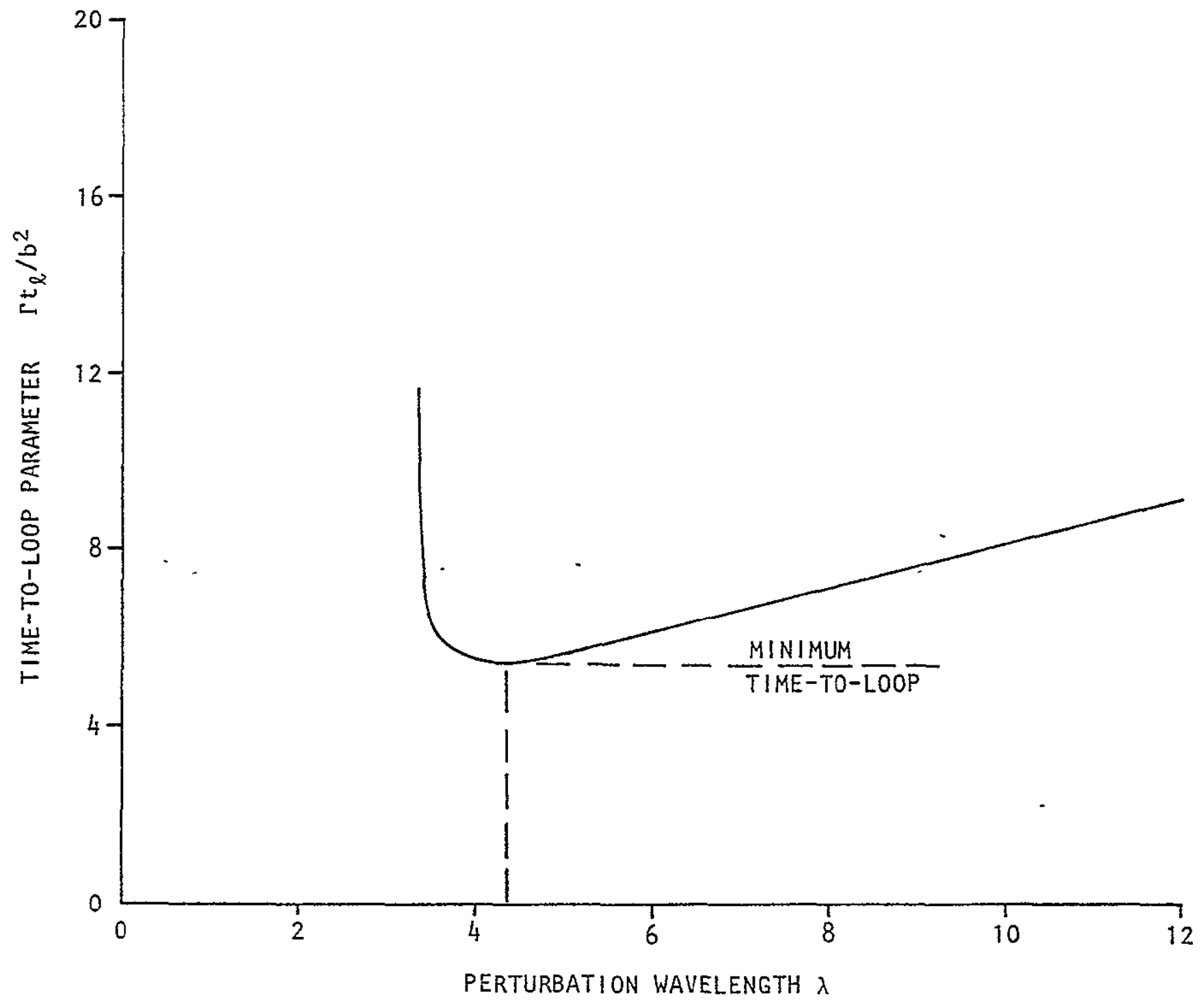

Figure 3.5 Dependence of Time-to-Loop Upon Perturbation Wavelength, $a_{x} / b=a_{y} / b=.125, \delta / b=.098$ 


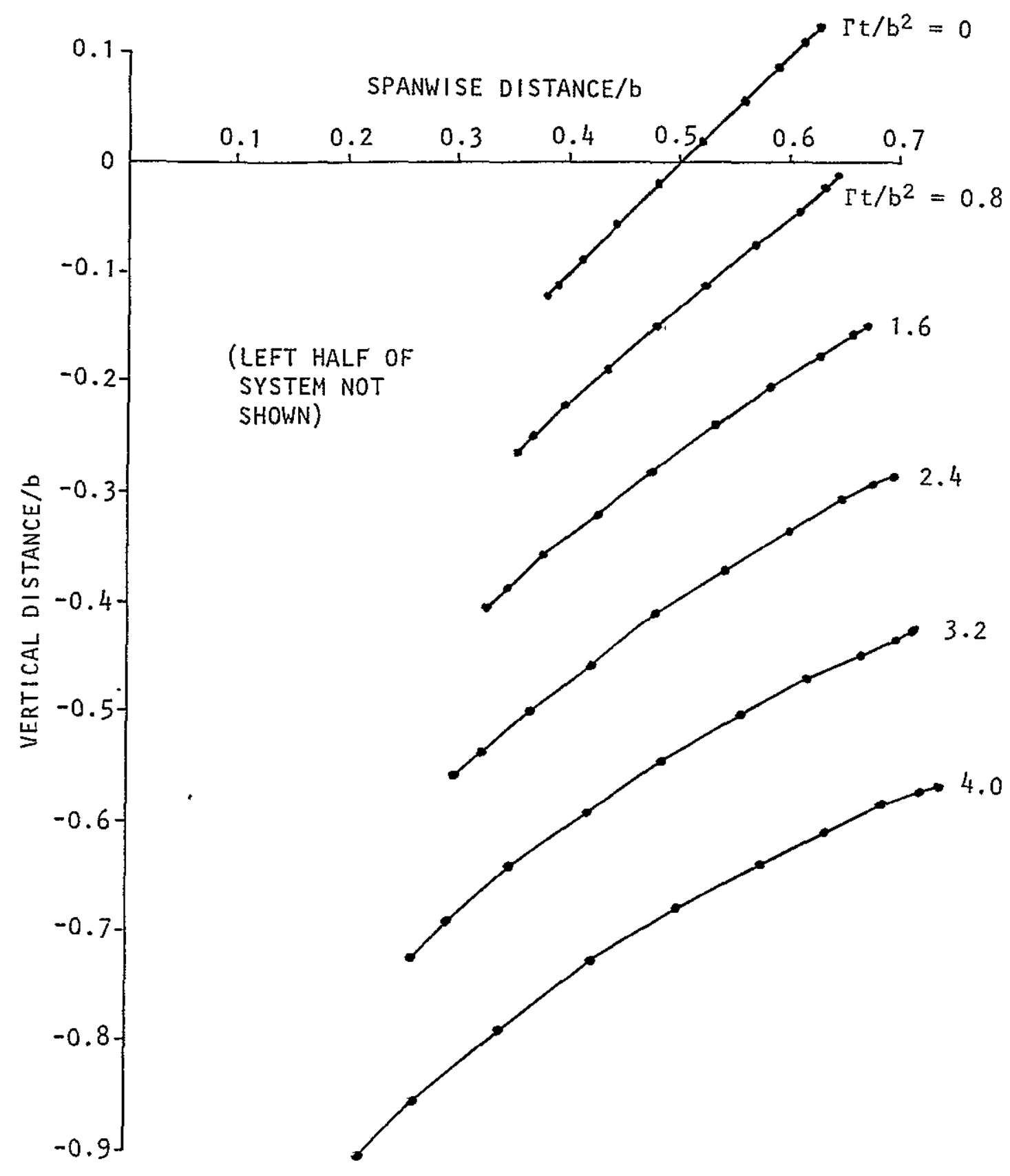

Figure 3.6 End-View of Vortex-Pair Distortion Toward Looping, $\lambda=5.0, \delta / b=0.098$ 


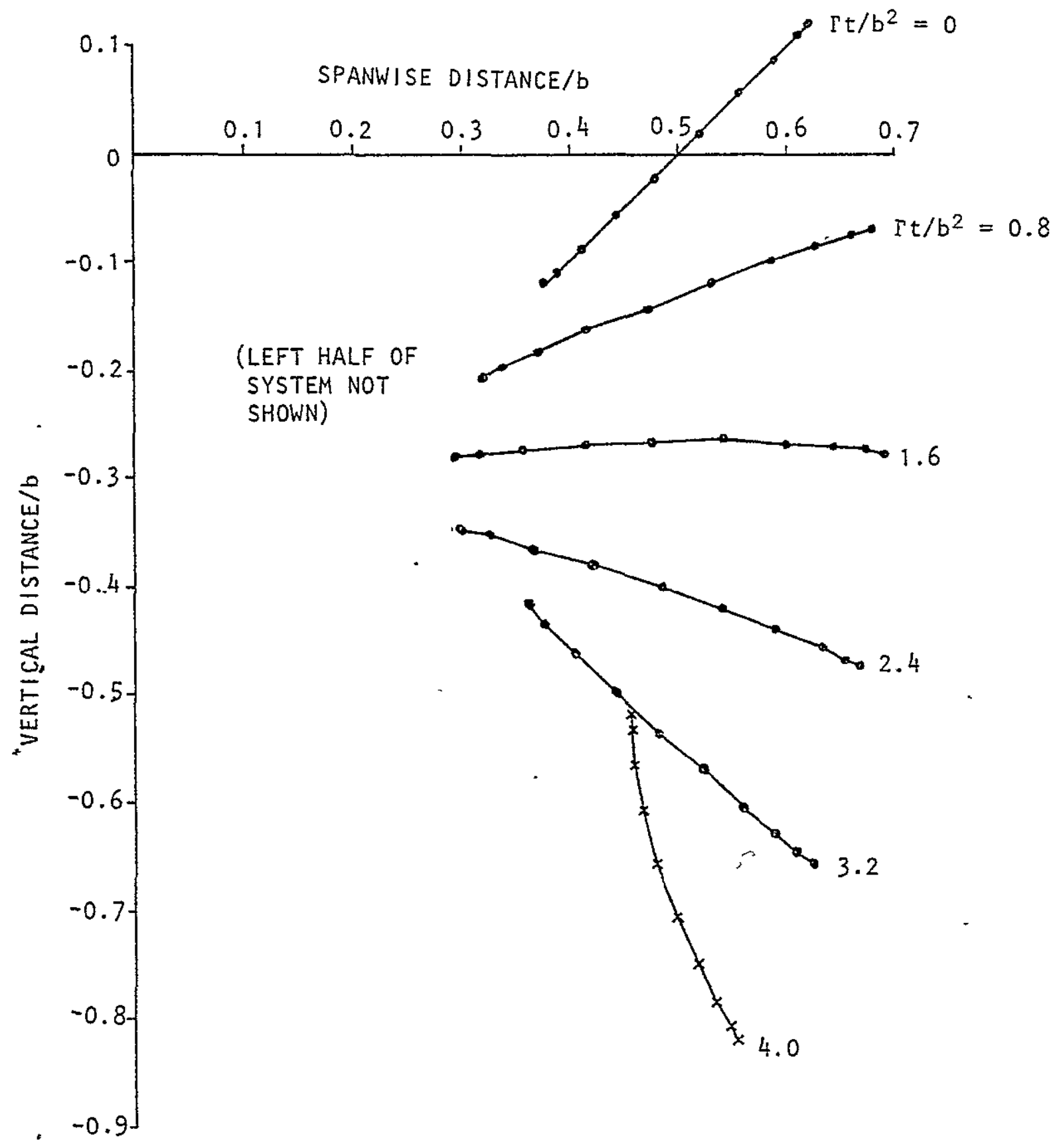

Figure 3.7 End View of Vortex Pair Distortion at Sub-Looping Wavelength, $\lambda=2.5, \delta / b=.048$ 


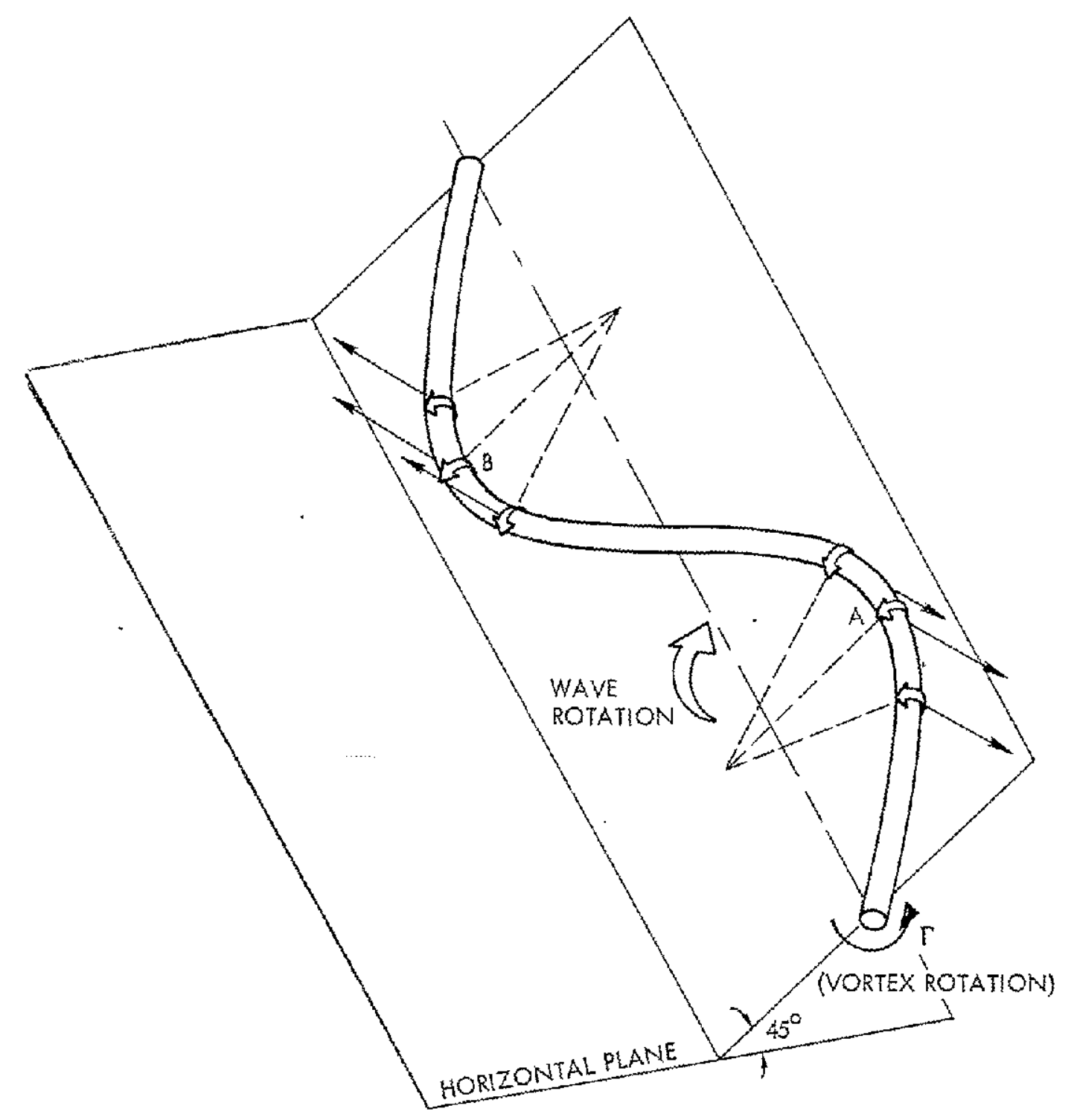

Figure 3.8(a) Mechanism for Opposingusense Vortex and Wave Rotations - Geometry 


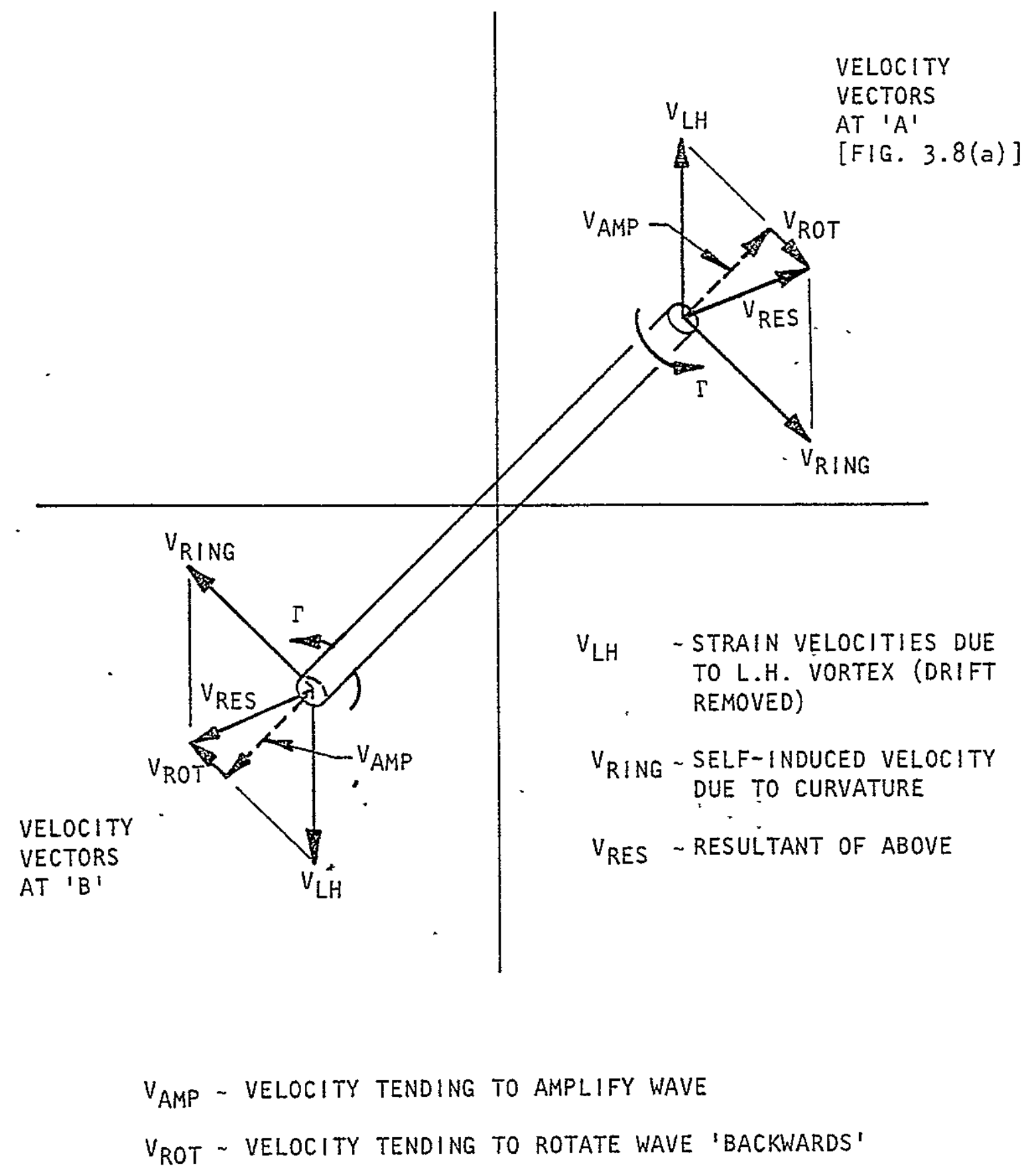

Figure 3.8(b) Mechanism for Opposing-Sense Vortex and Wave Rotations - Velocities 


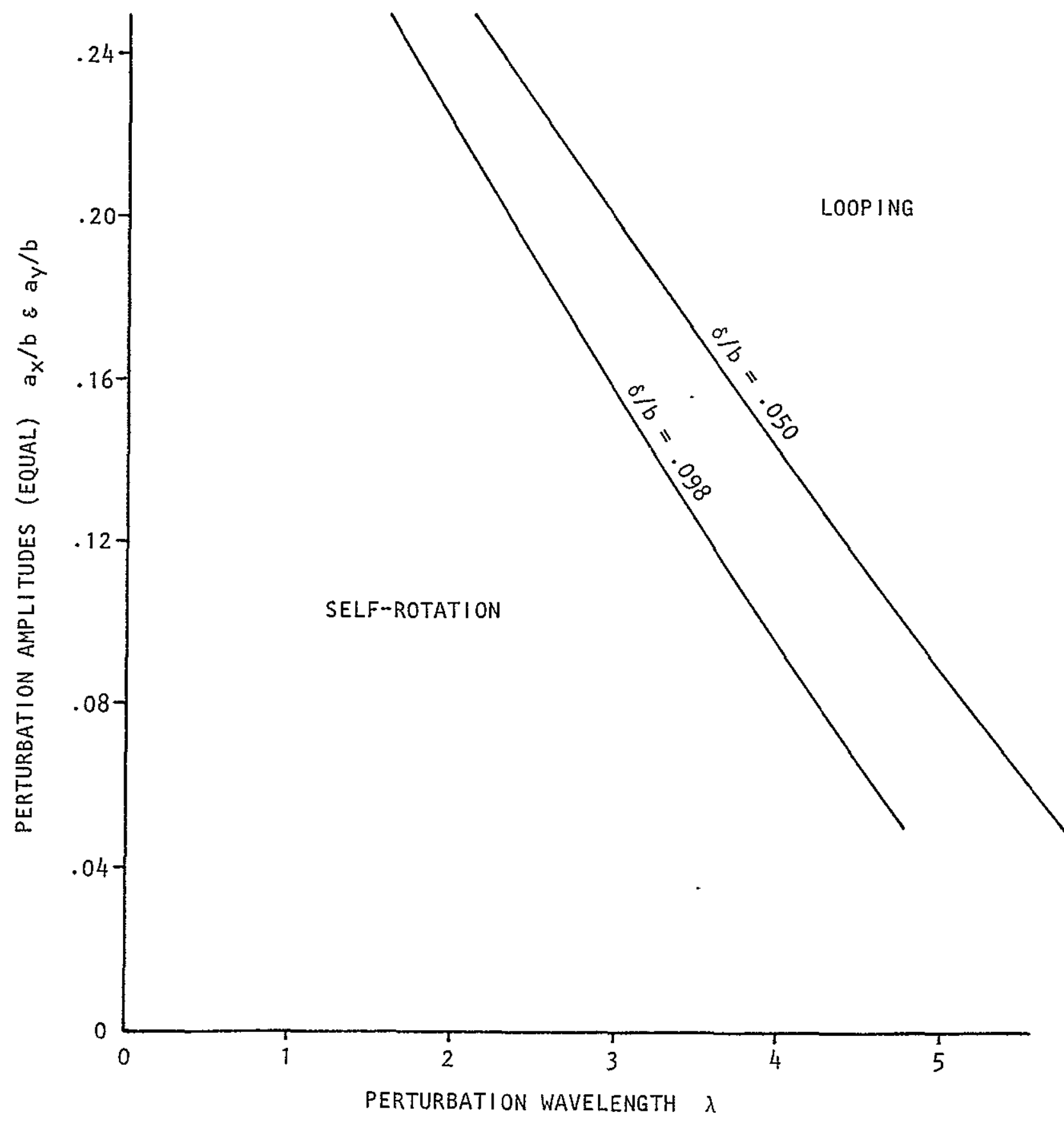

Figure 3.9 Lower Limit Wavelengths for Vortex Looping to Occur 


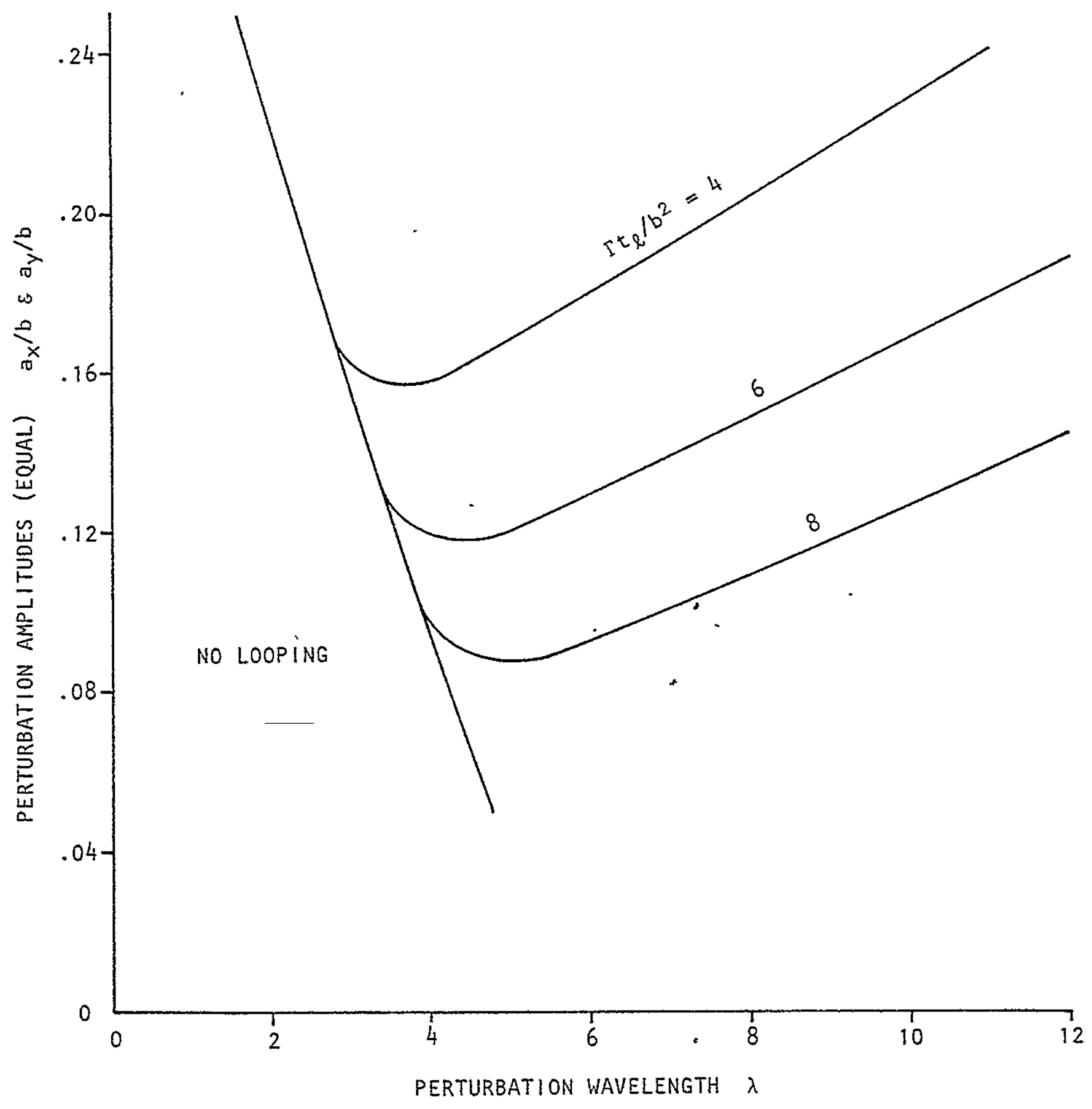

Figure 3.10 Time-to-Loop as a Function of Perturbation Wavelength and Initial Amplitude, $\delta / b=.098$ 


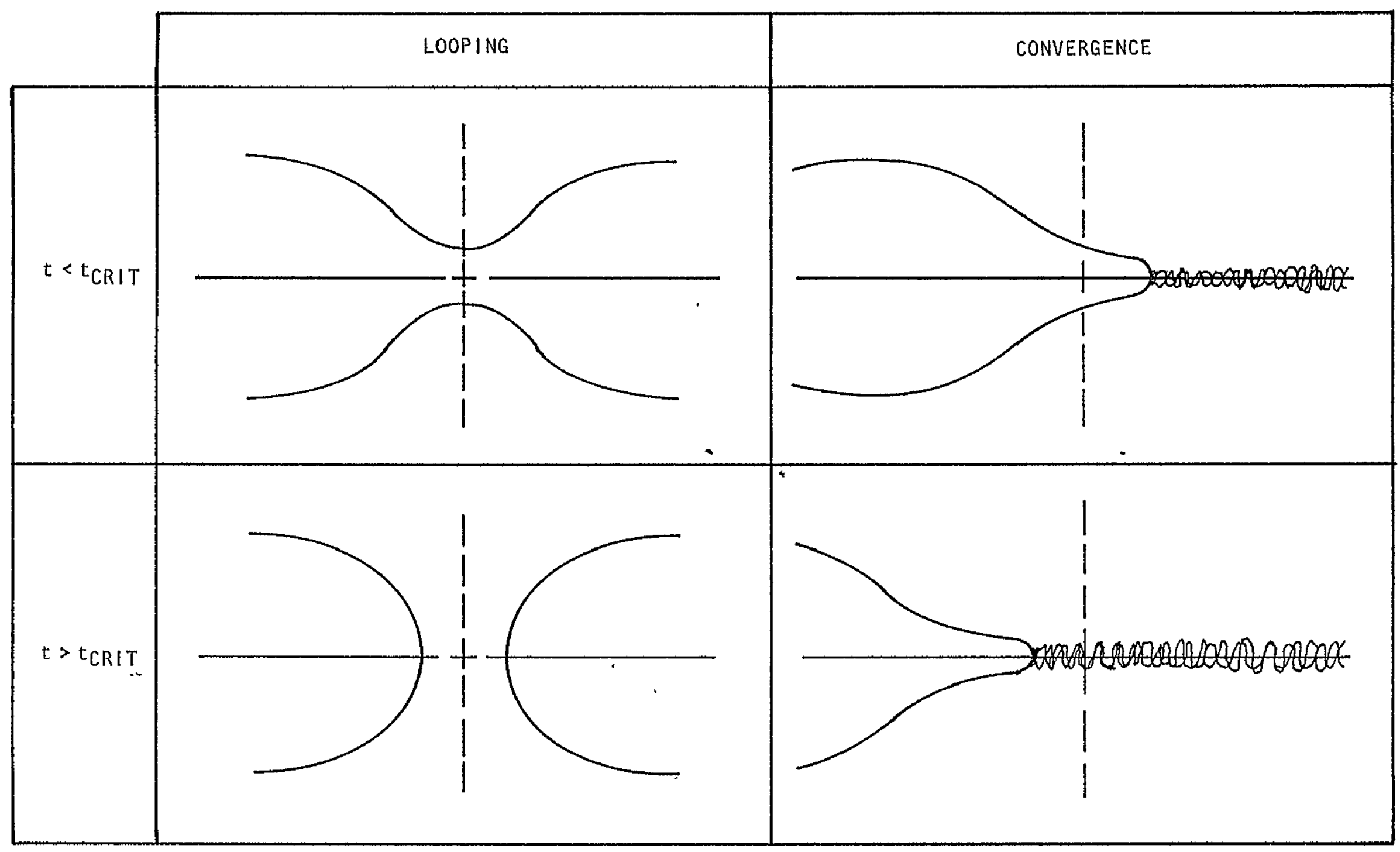

Figure 4.1 The Distinction Between Vortex "Looping" and "Convergence" 


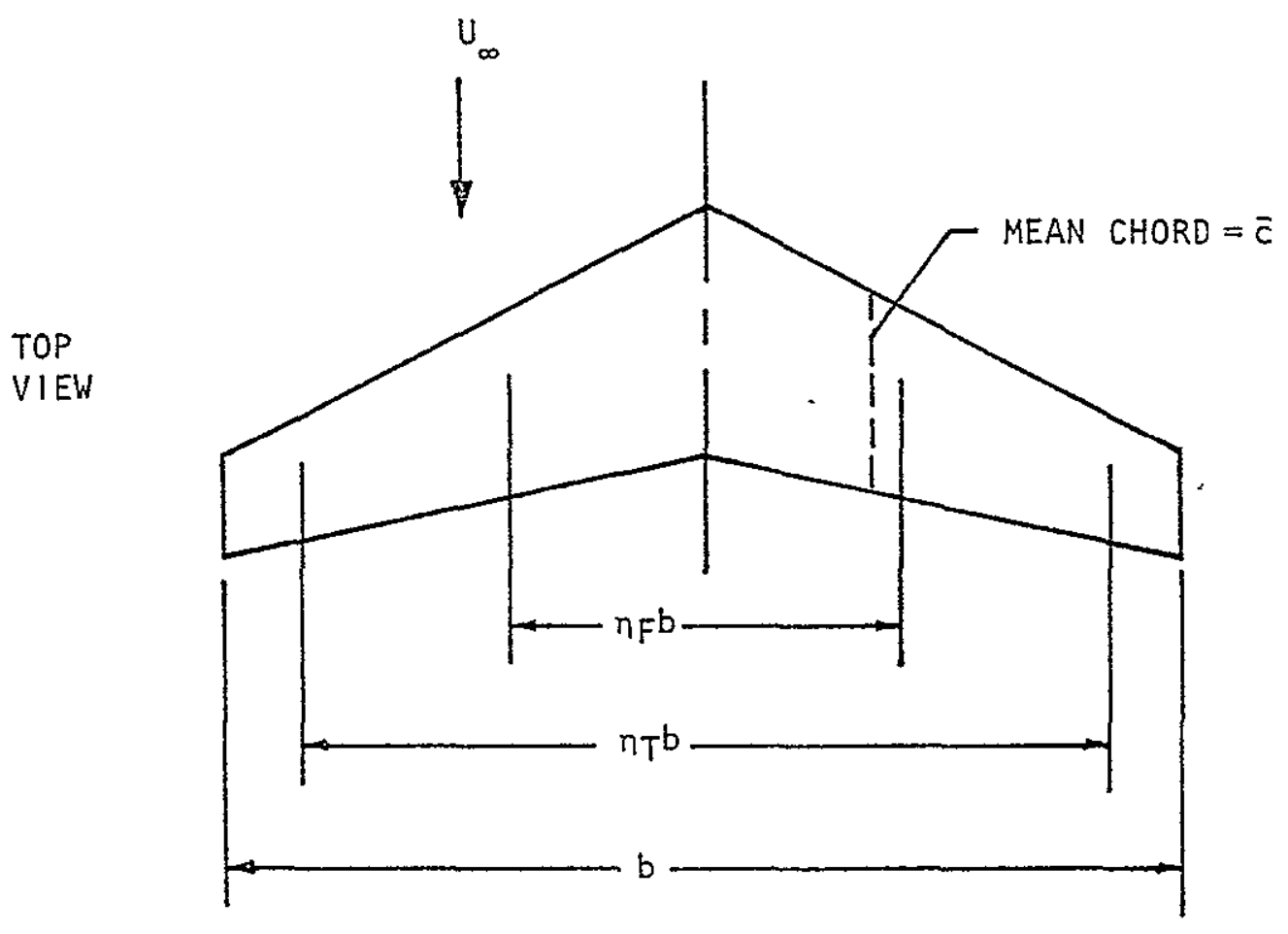

AFT

VIEW

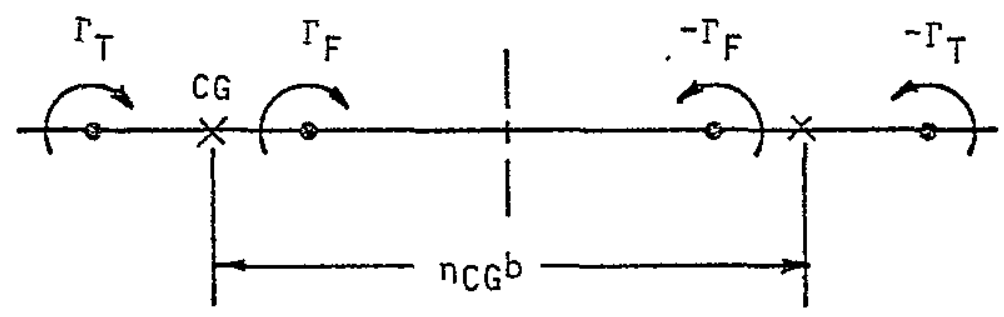

WHERE

$$
n_{C G}\left(\Gamma_{F}+\Gamma_{T}\right)=n_{F} \Gamma_{F}+n_{T} \Gamma_{T}
$$

Figure 4.2 Kinematic Variables for Twin Pair Studies - 


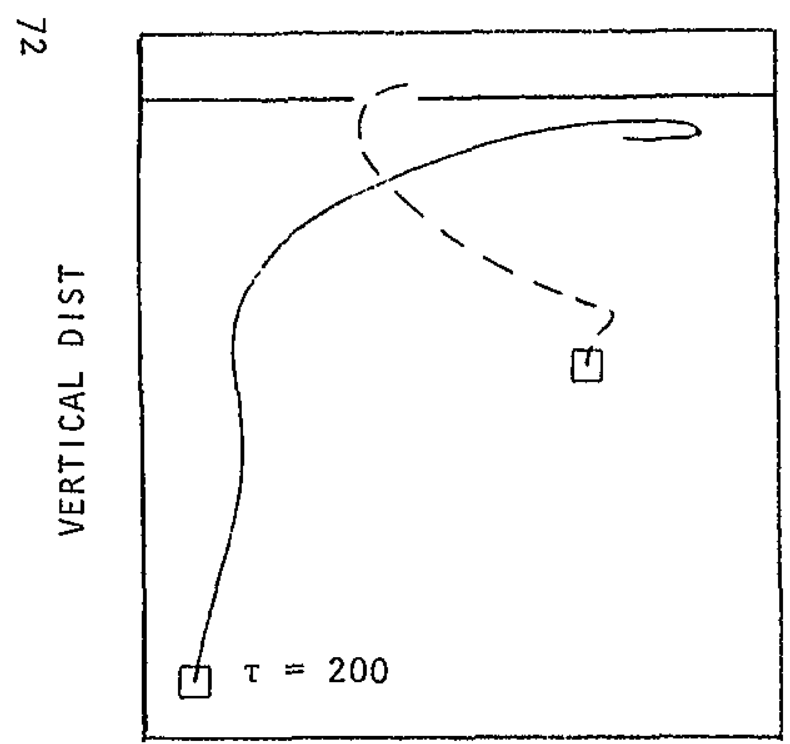

SPANWISE DIST

(a) $n_{F}=0.2$

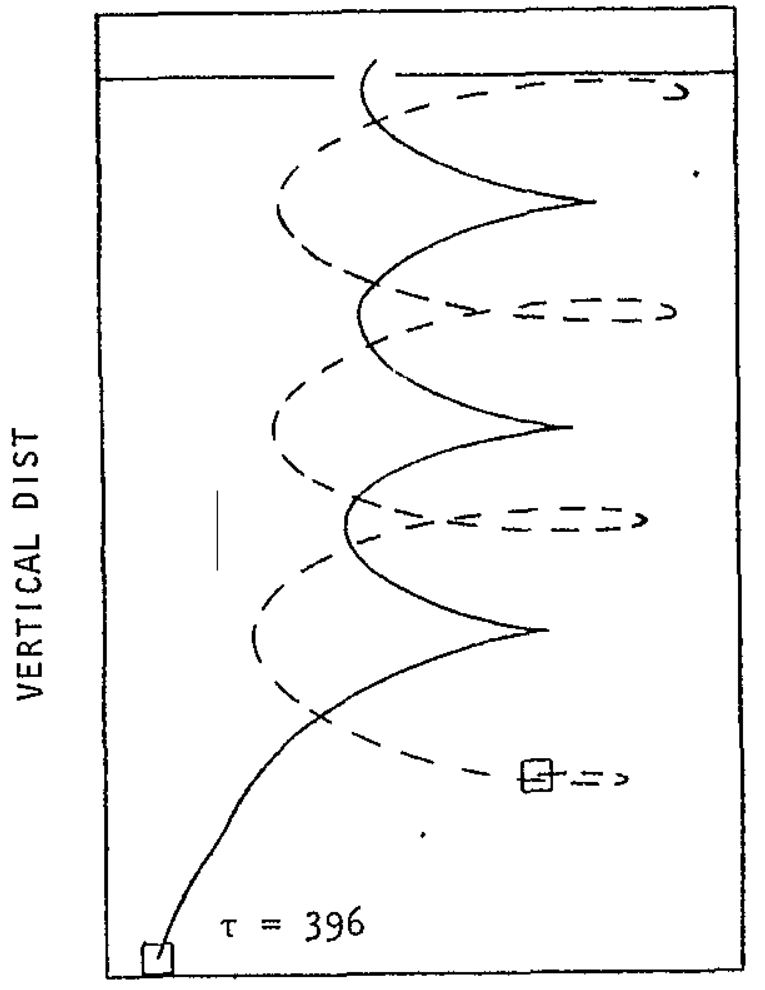

SPANWISE DIST

(b) $n_{F}=0.333$

NOTE: VERTICAL SCALE COMPRESSED BY A FACTOR OF 5 .

Figure 4.3 Cross-Sectional Trace for the Convergence Point on Twin Vortex Systems, as a Function of $n_{F}\left(\Gamma_{T} / \Gamma_{F}=0.60, n_{T}=1.0\right)$

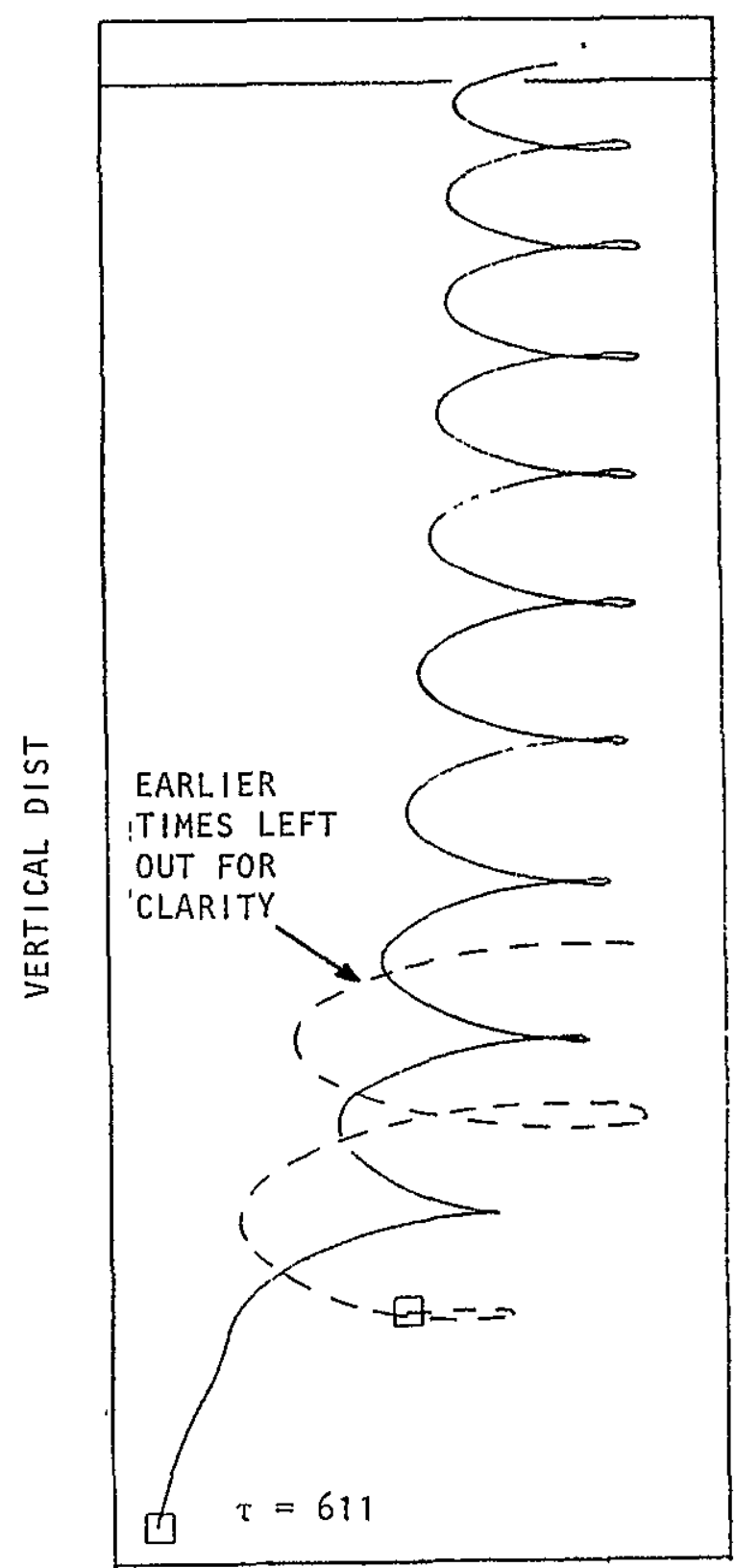

SPANWISE DIST

(c) $n_{F}=0.556$ 


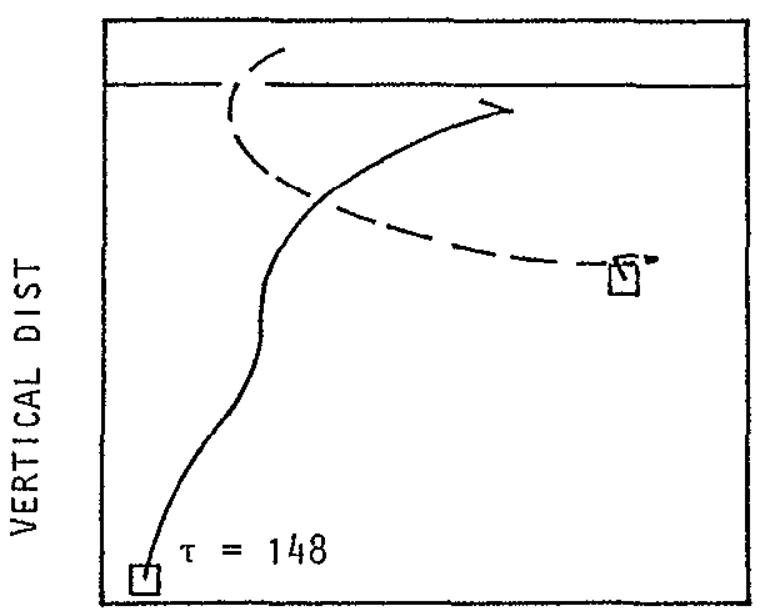

SPANWISE DIST

(a) $\Gamma_{\mathrm{T}} / \Gamma_{\mathrm{F}}=0.6$

NOTE: VERTICAL SCALE COMPRESSED BY A FACTOR OF 5.

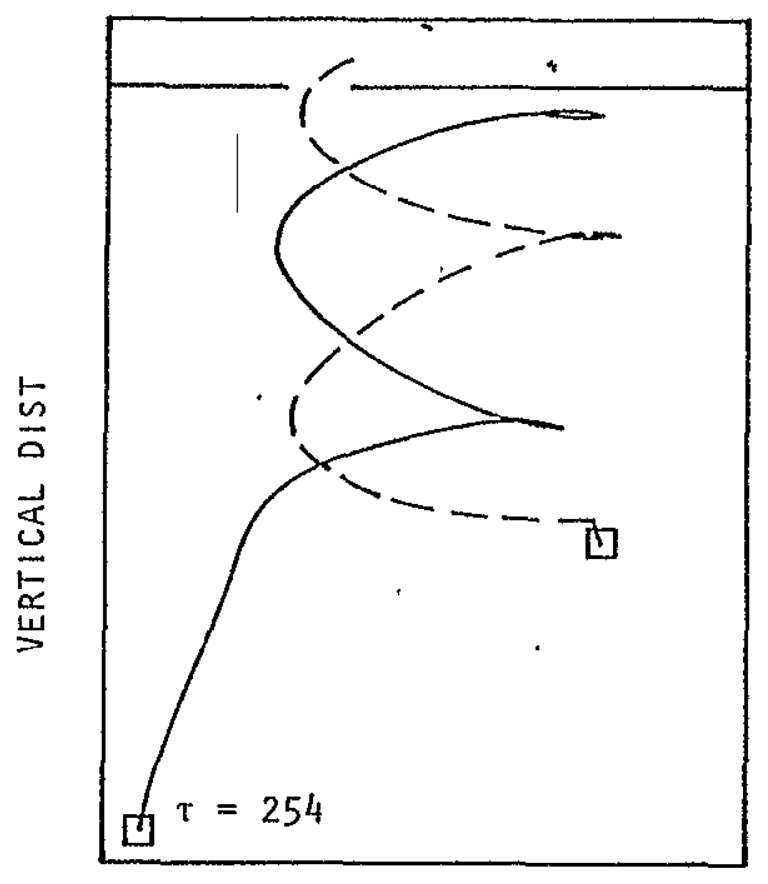

SPANWISE DIST

(b) $\Gamma_{T} / \Gamma_{F}=1.0$

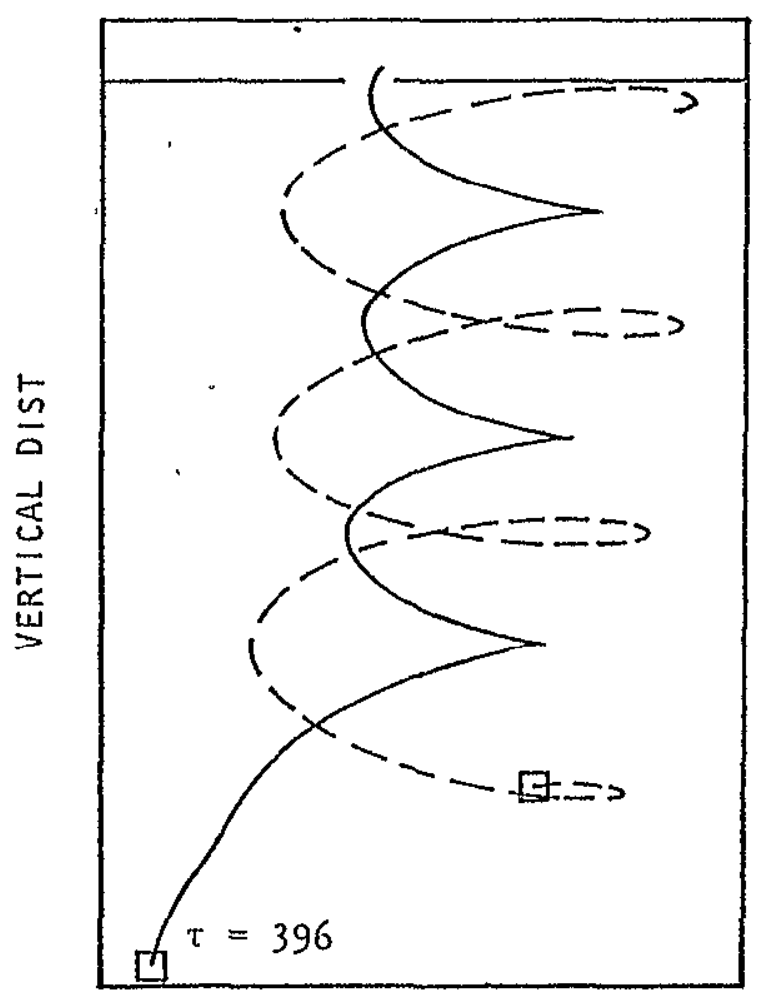

SPANWISE DIST

(c) $\Gamma_{T} / \Gamma_{F}=1.667$

Figure 4.4 Cross-sectional Trace for the Convergence Point on Twin Vorlex systems as a Function of $\Gamma_{T} / \Gamma_{F}\left(n_{F}=0.333, n_{T}=1.0\right)$ 
POSITION

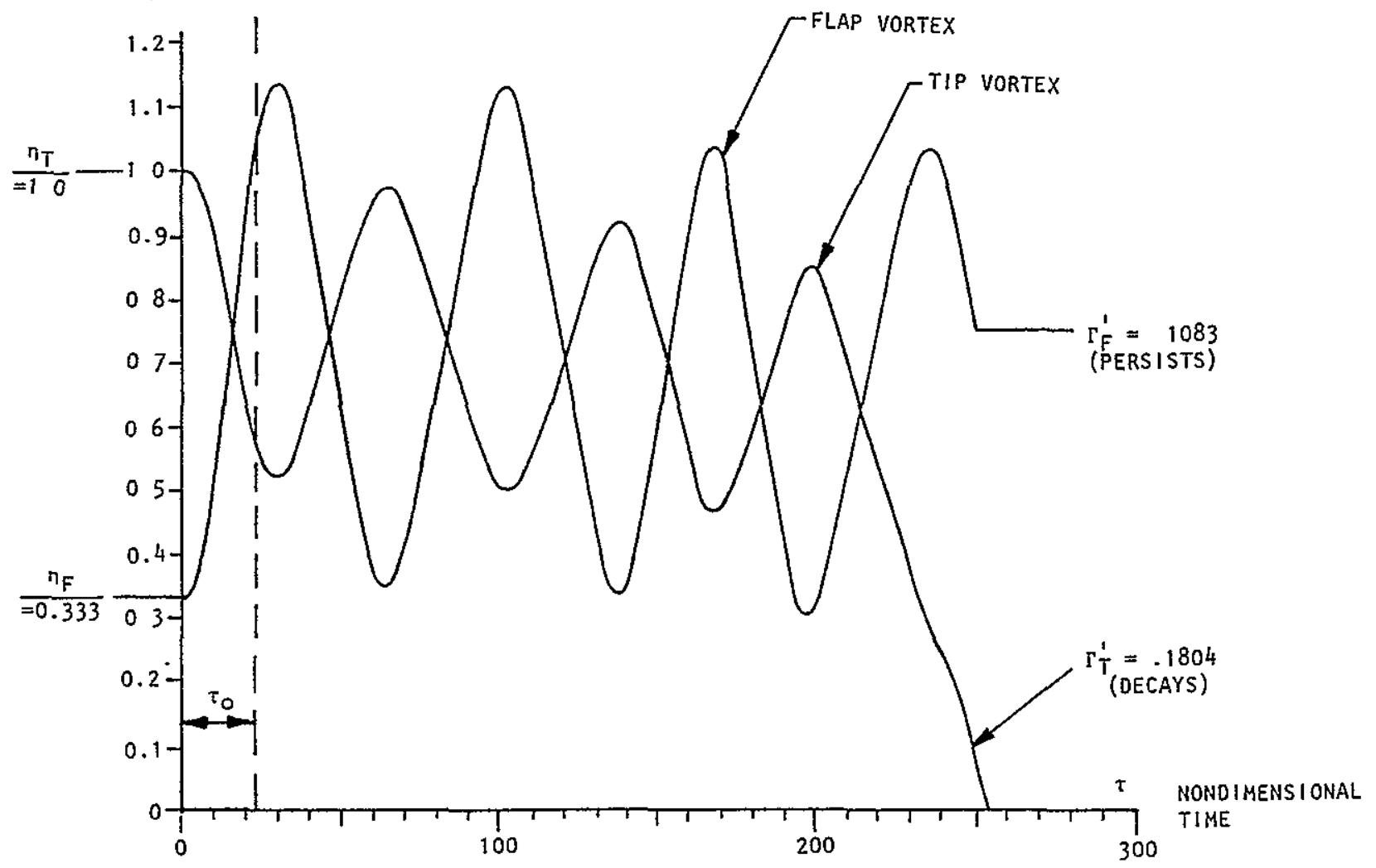

SPANWISE

POSITION

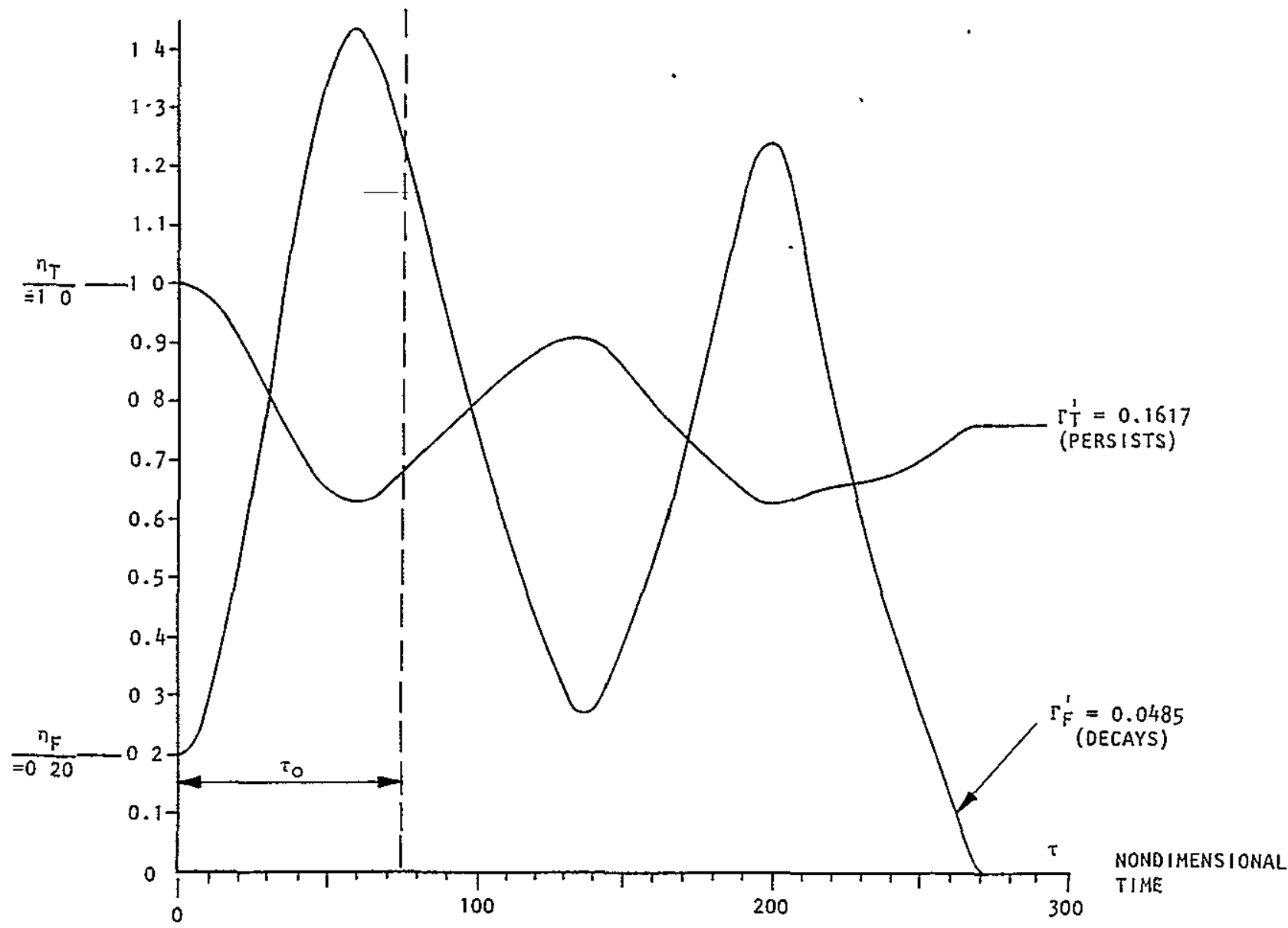

Figure 4.5 Examples of Tip- and Flap-Vortex Convergence 


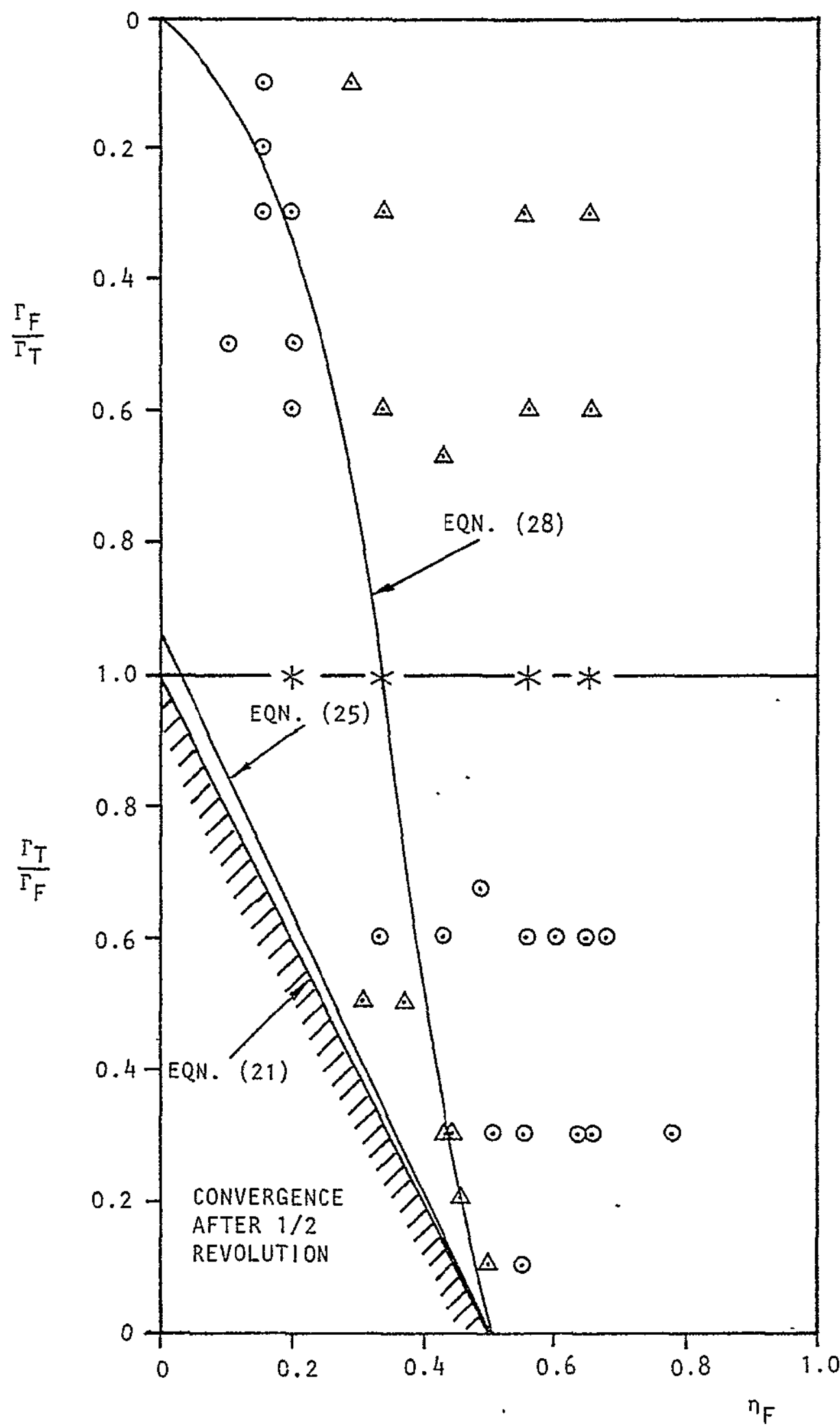

$\odot$ FLAP CONVERD

$\triangle$ TIP CONVERG

* EQUAL CHANCB

Figure 4.6 Twin-Pair Parametric Study: Identification of the Convergent Vortex 


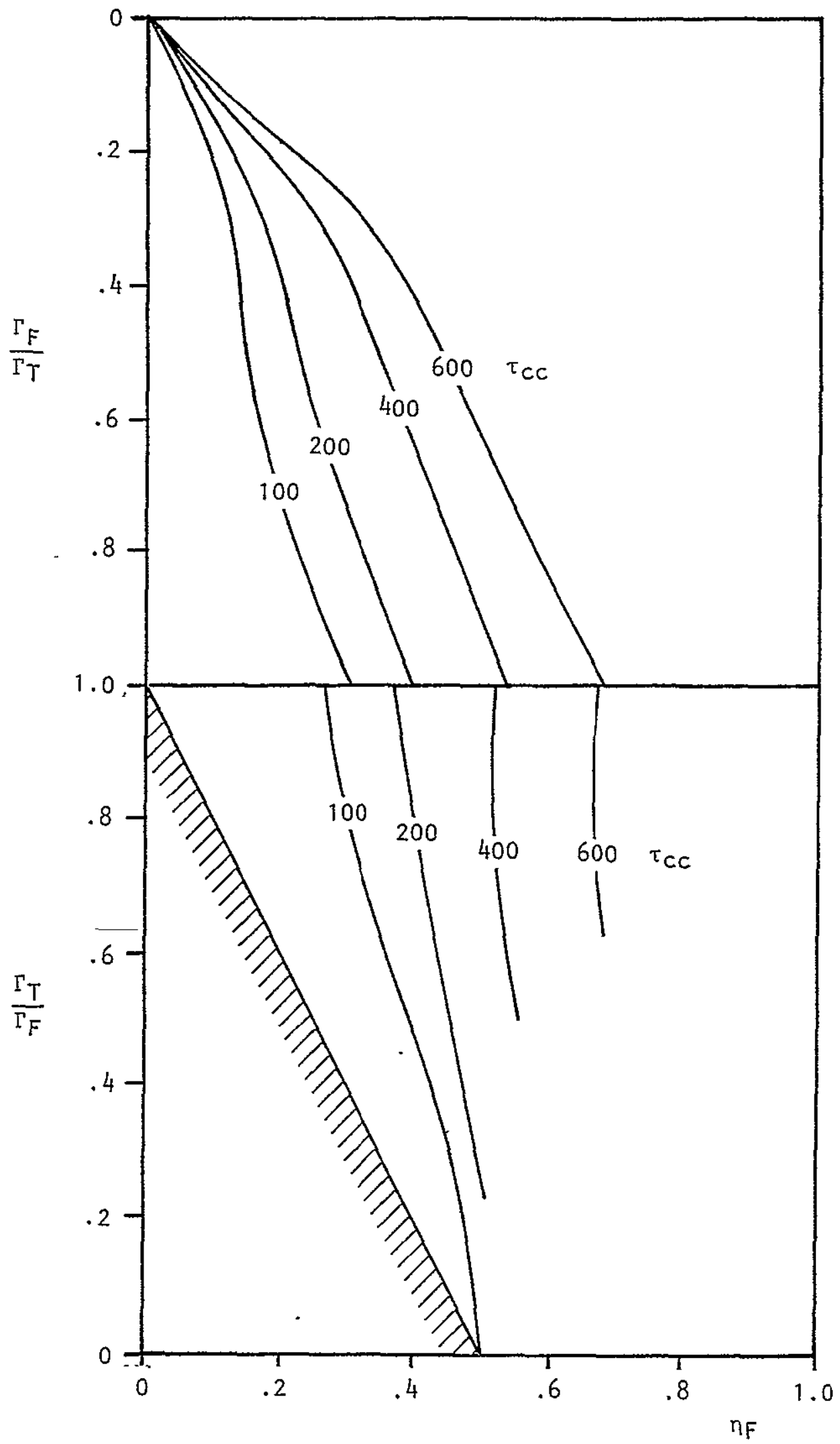

Figure 4.7a Twin Pair Parametric Study: Time-to-Converge $\left(C_{L} / A R=.1714, \lambda=5.375, \delta / b=.098\right)$ 


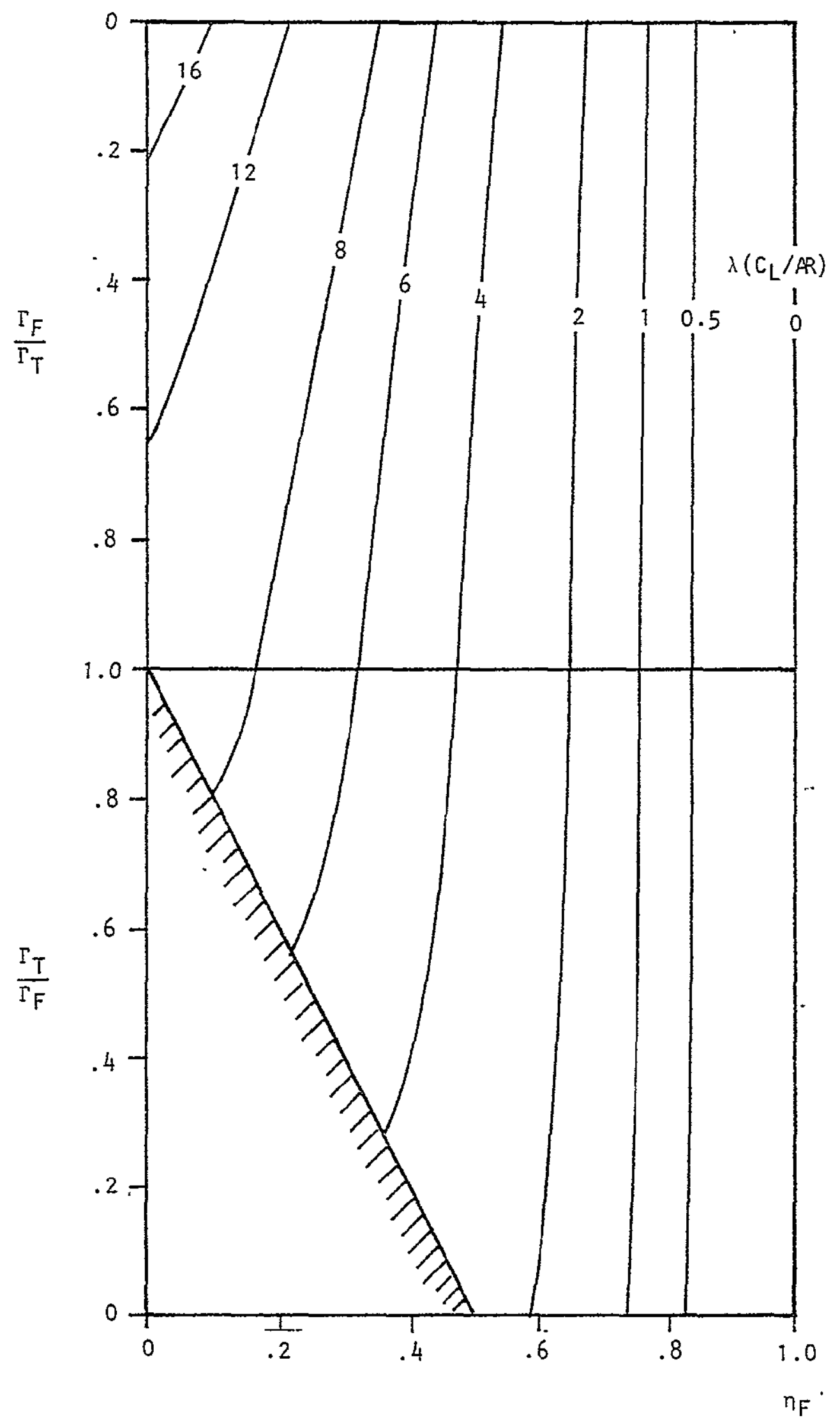

Figure 4.7b Twin Pair Parametric Study: Wavelength/Loading Parameter 


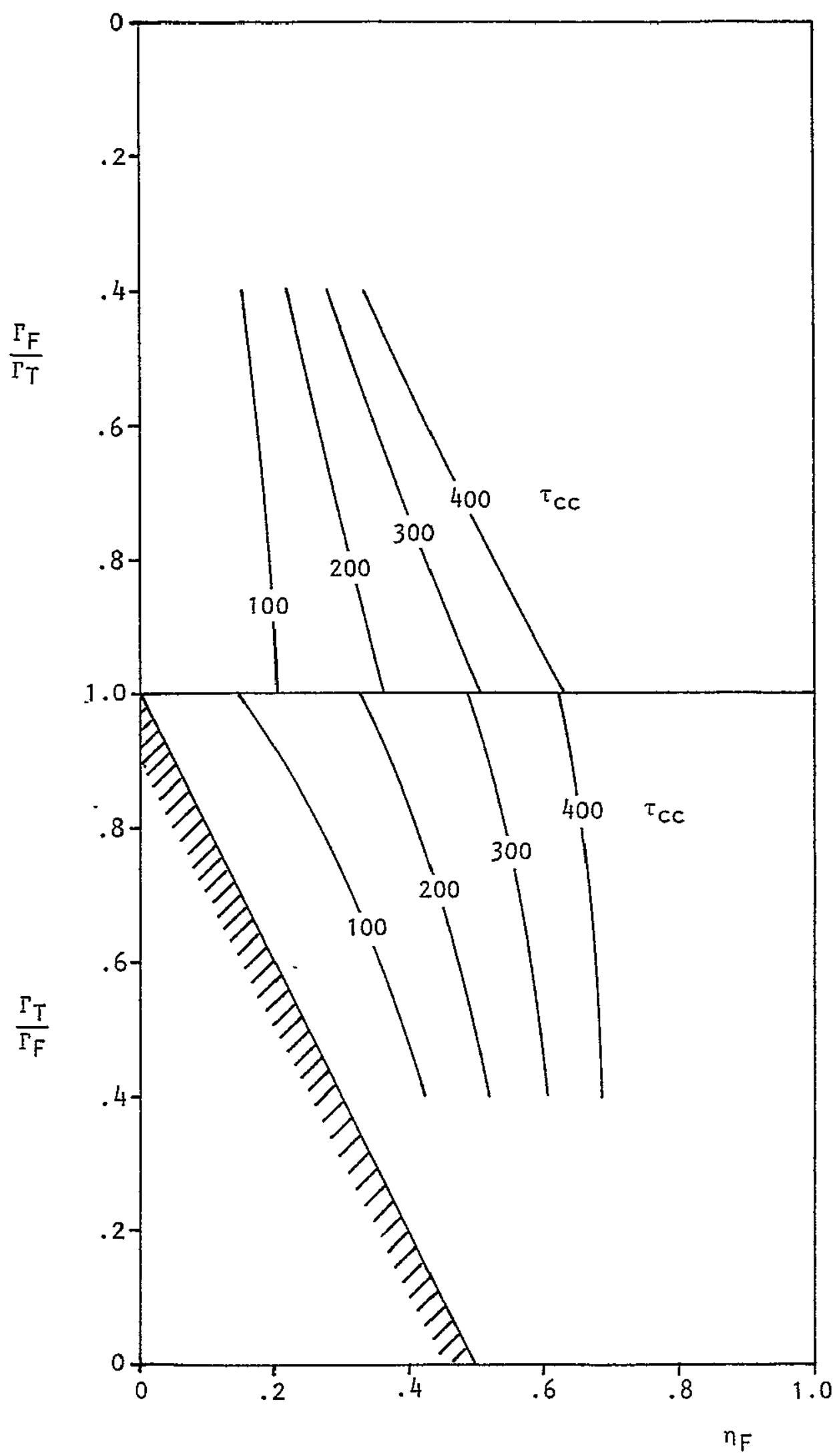

Figure 4.8 Twin Pair Parametric Study: Time-to-Converge $\left(C_{L} / A R=0.25\right.$, Correct $\left.\lambda, \delta / b=0.98\right)$ 


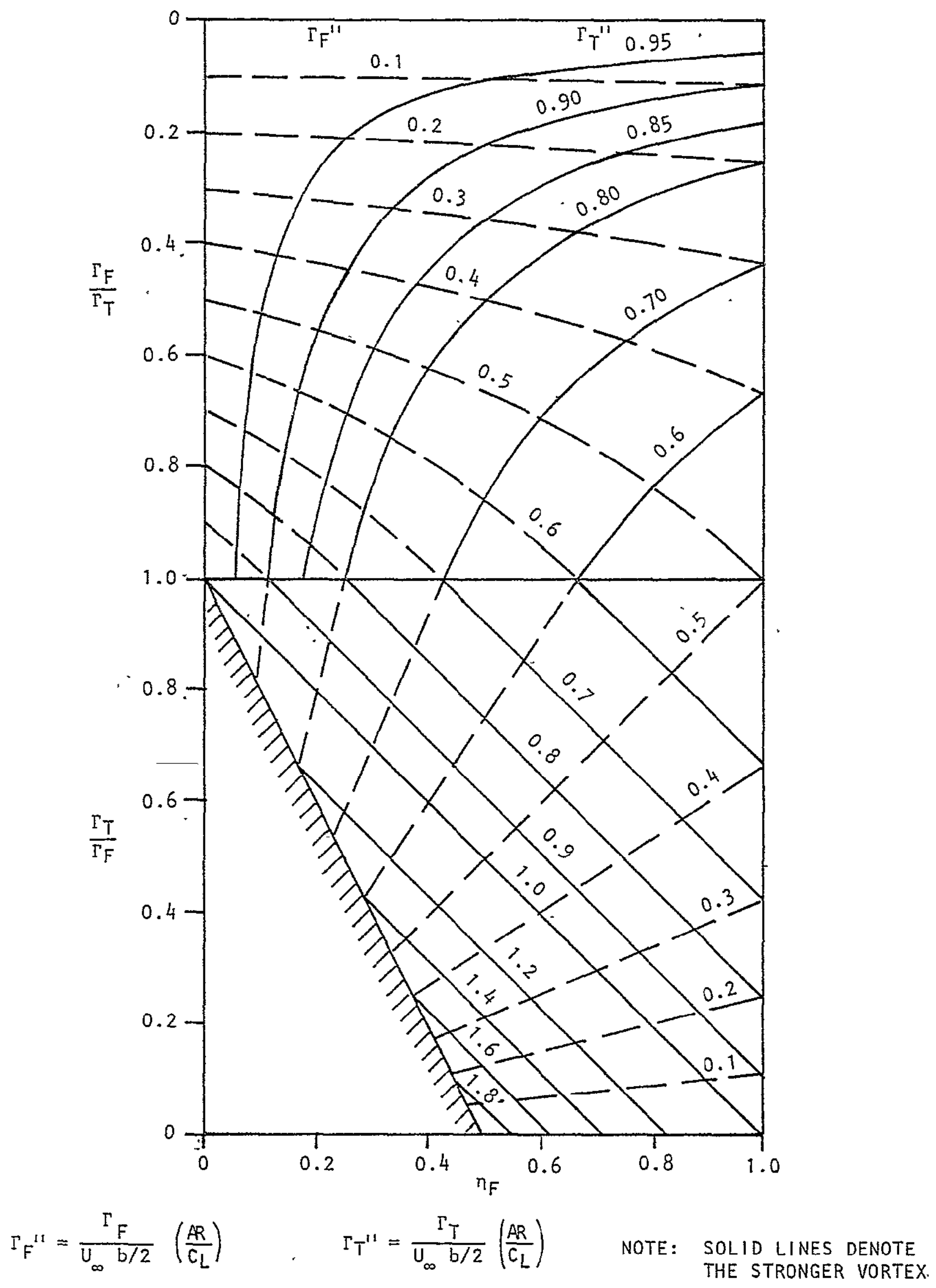

Figure 4.9 Strength of the Vortex Remaining Aftèr Convergence 


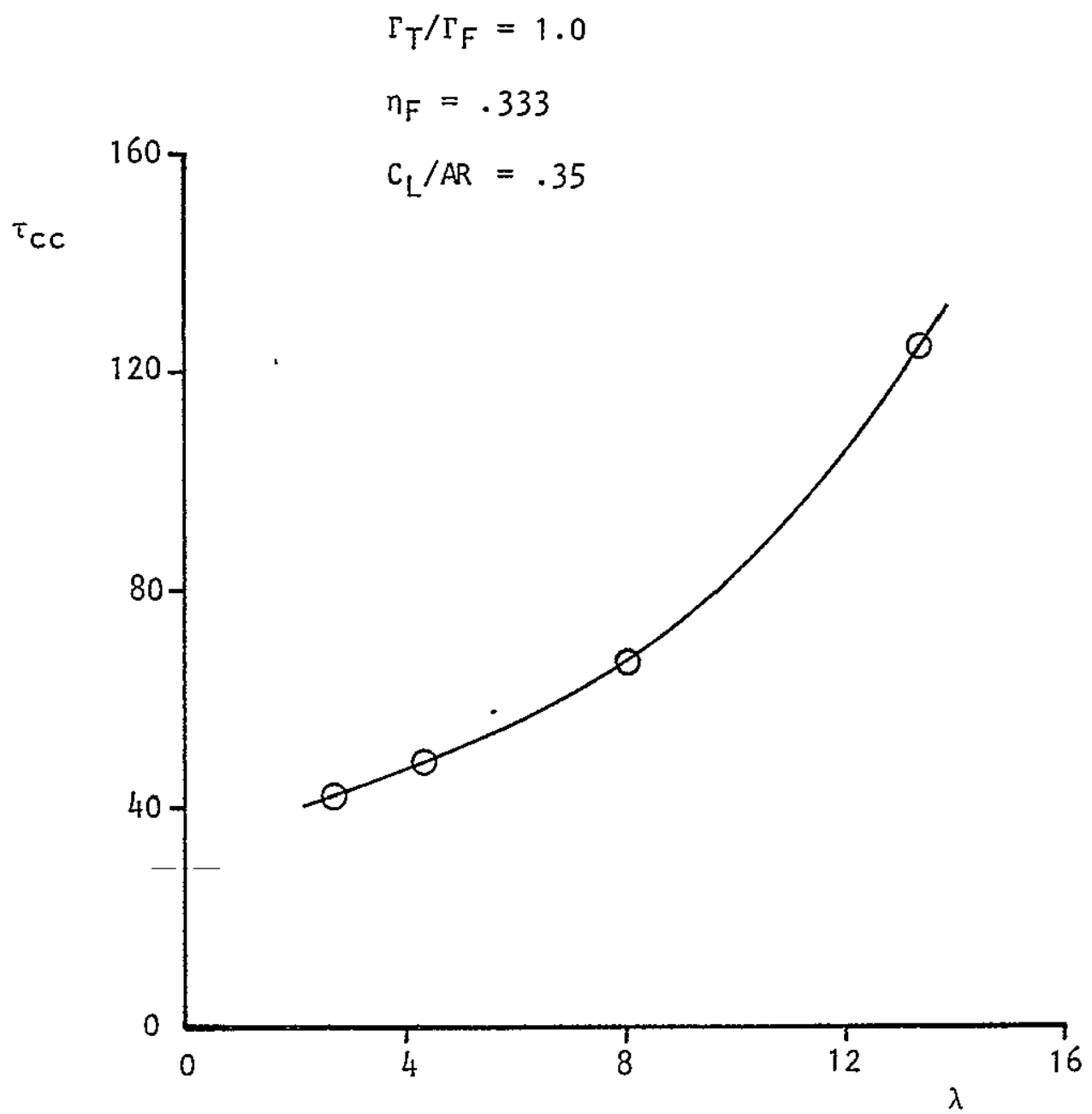

Figure 4.10 Effect of Spiral Wavelength on Time-to-Converge 


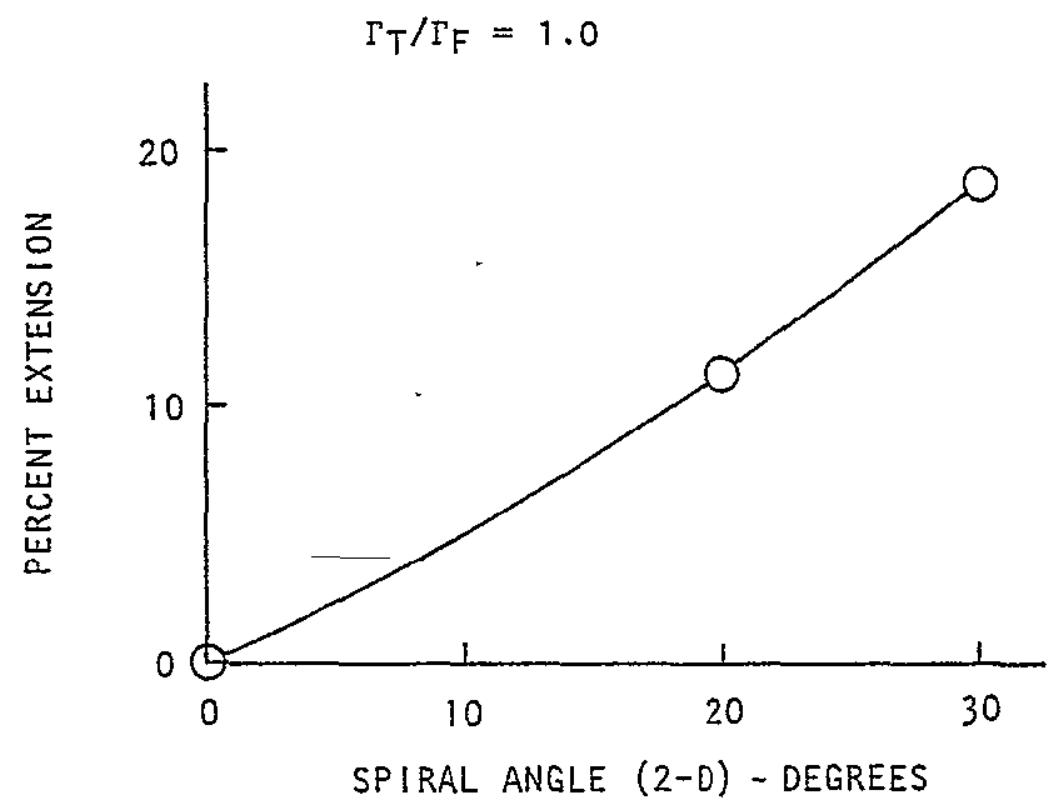

Figure 4.11 Three Dimensional Extension of Twin Spiral Waves 


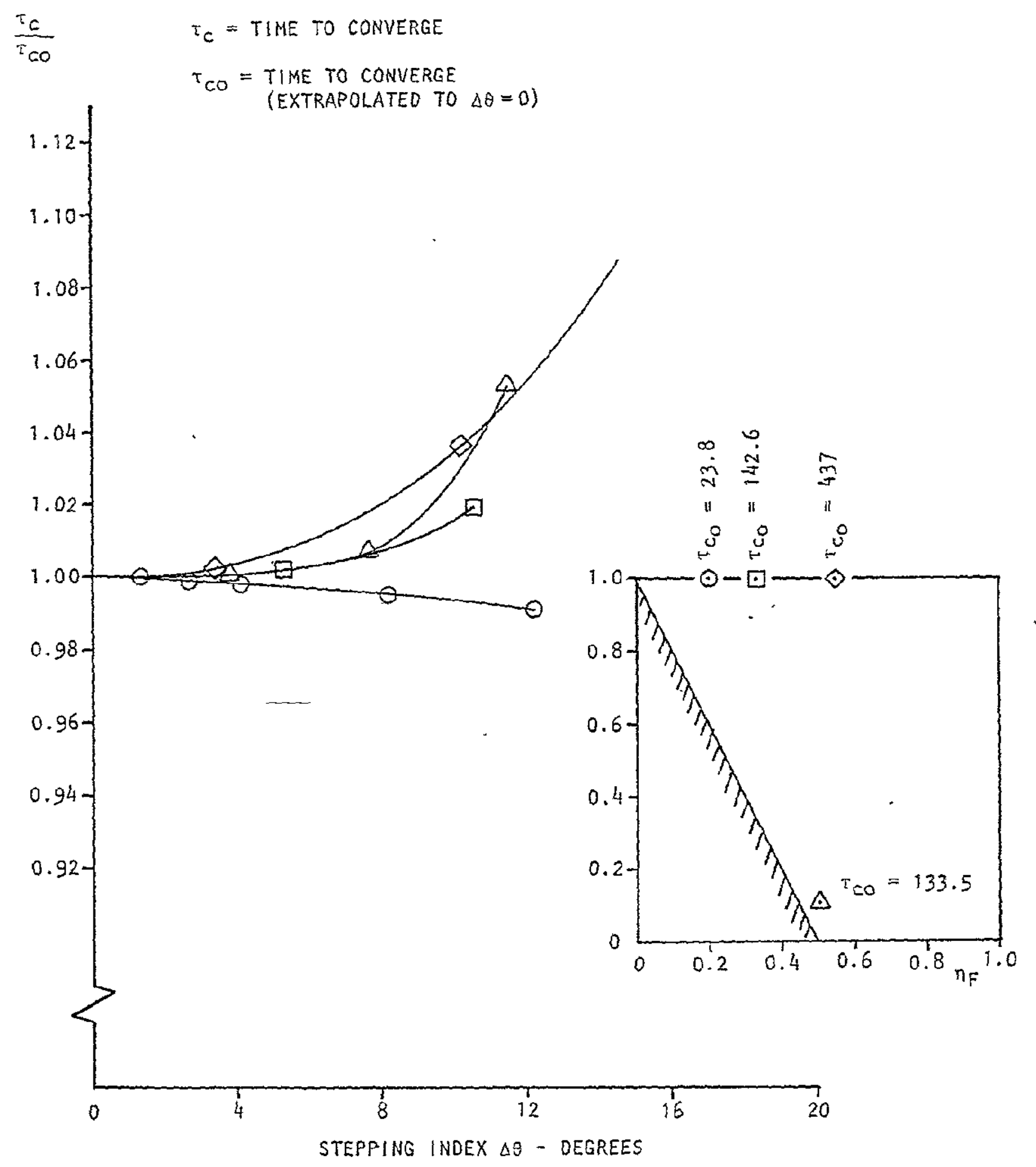

Figure 4.13 Effect of Integration Step-Size on Time-to-Converge

$$
C_{L} / A R=.1714 \quad \lambda=5.375
$$


$D_{50}=$ DIST. BETWEEN VORTICES AT $\tau=50$ (2-D CALC.)

$D_{0}=$ DIST. BETWEEN VORTICES AT $\tau=0$

NOTE: EFFECTS OF REFLECTION ACROSS CENTER PLANE NOT INCLUDED

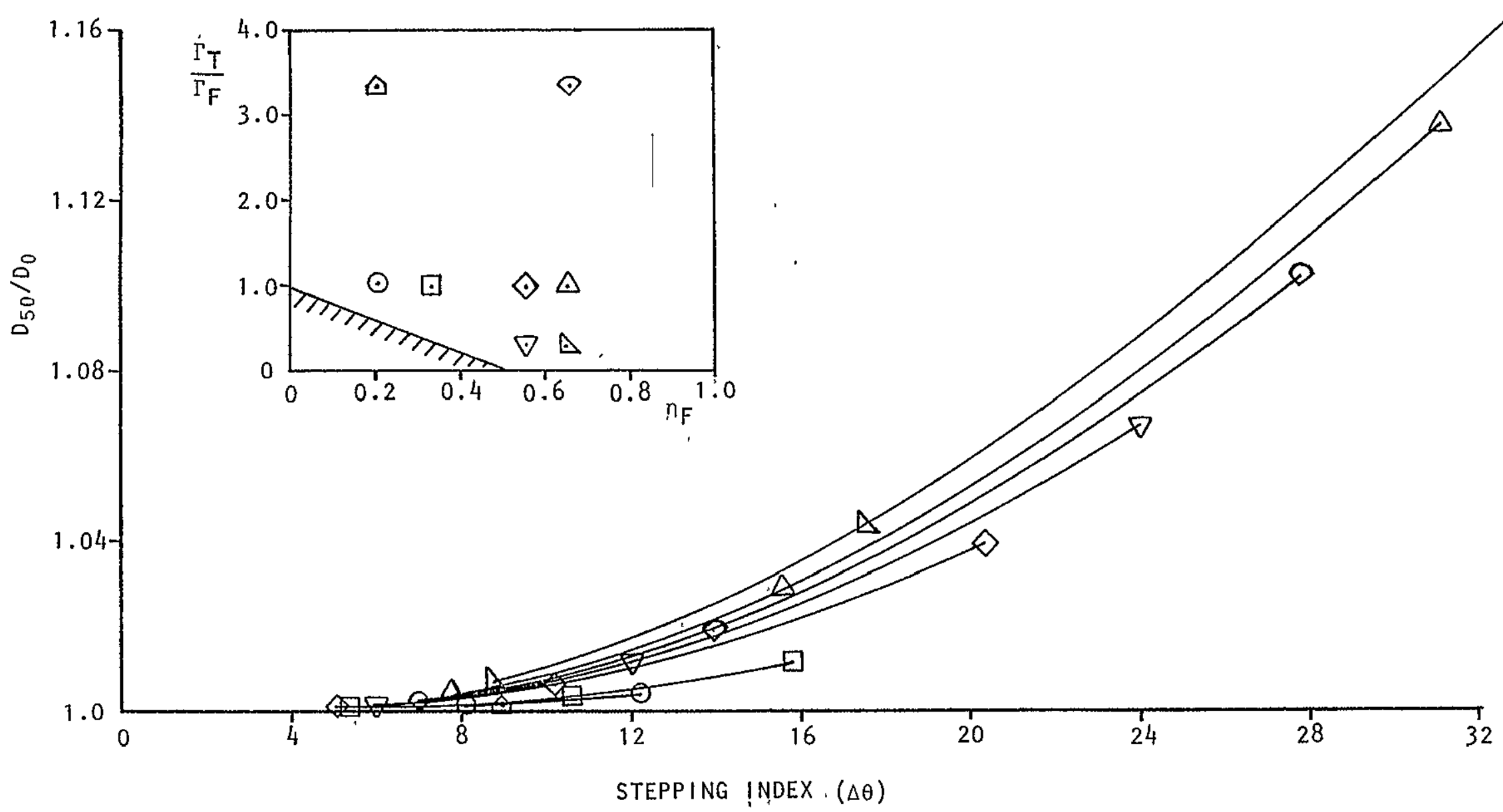

Figure 4.14 Effect of Integration Step-Size on Vortex Spacing 

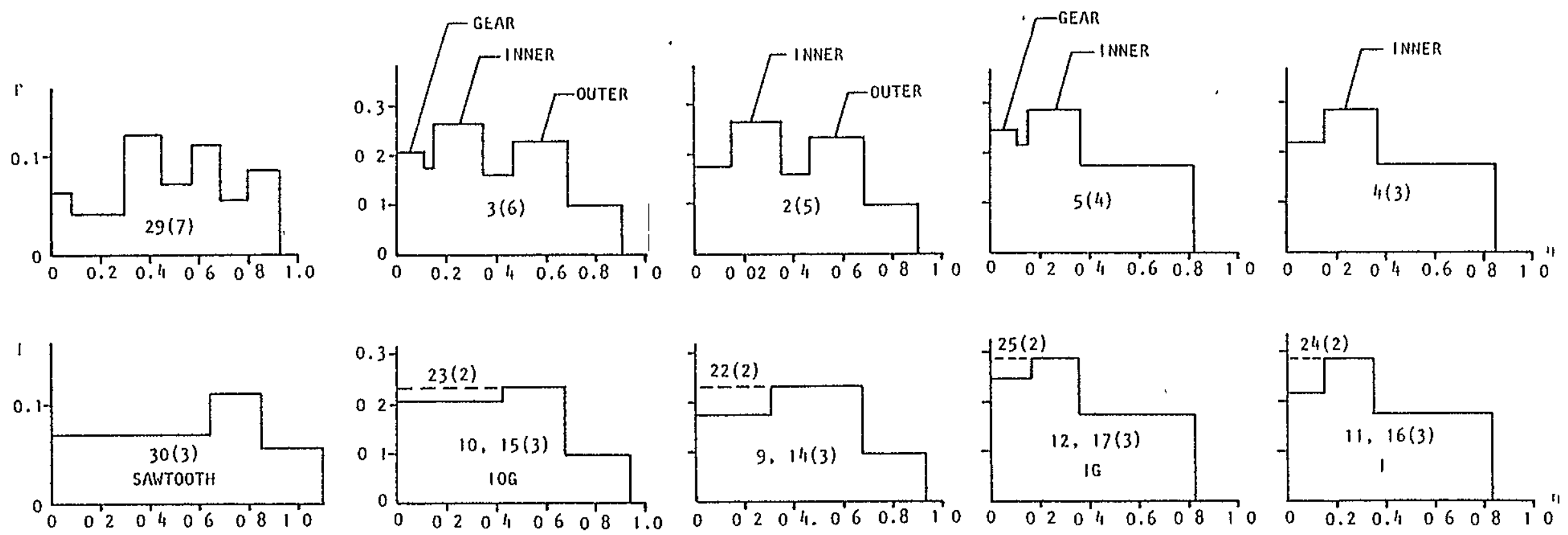

(a) CV9go, SAUTOOTH

(b) B7'17, FULL CONFIGURATION

(c) B717, INNER-PLUS-OUTER

(d) 8747 , INNER-PLUS-GEAR

(c) B747, INNER ONLY

RUNS $2,3,4,5,29$ 2-0 INITIALIZATION RUNS $9,10,11,12,24,3-D$ RELAXATION, A/C FIXED RUNS $14,15,16,173$ PAIRS, SPACE FIIXED RUNSS $22,23,24,25$ PAIRS, SPACE FIXED FIGURES IN PARENTIESES SHOW NUMBER OF PAIRS

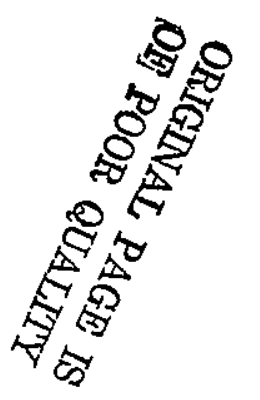
FIGURES WITHOUT PARENTHESES SHOW RUN NUMBCR

Figure 5.1 Span Load and Run Definition for Multiple-Pair Cases 


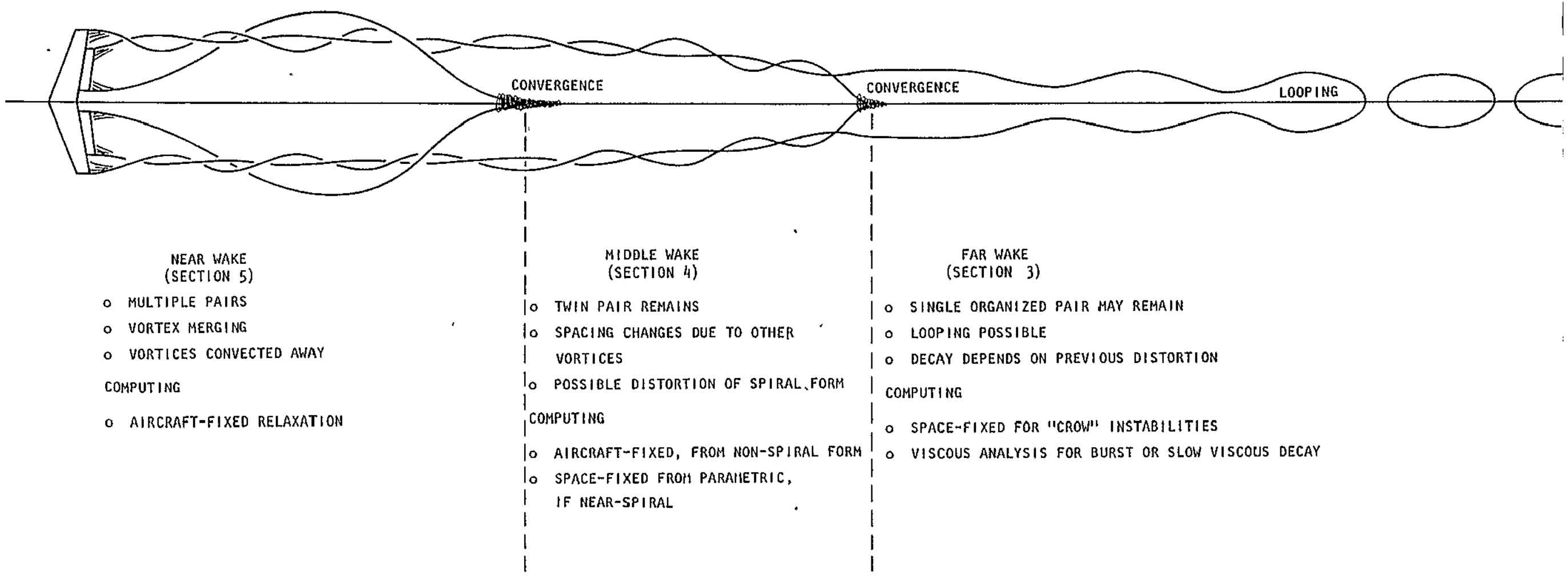

NOTE. IN THE ABOVE SKETCH THE AXIAL DIMENSION IS FORESHORTENED AND THE NUMBER OF WAVES IS REDUCED.

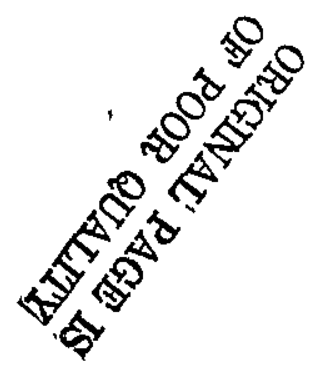

Figure 5.2 Calculation Regions for a Multiple-Pair Vortex Wake 

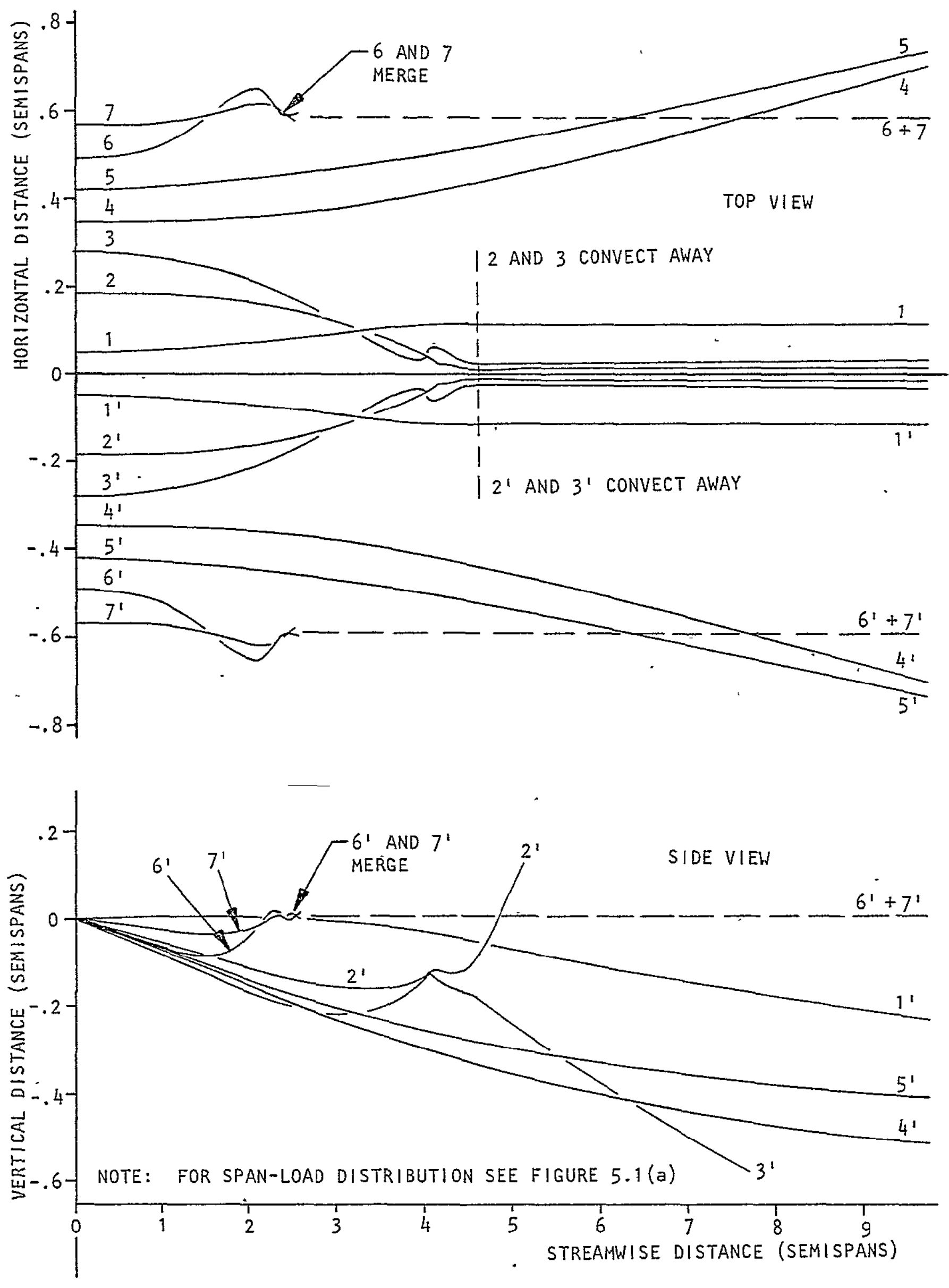

Figure 5.3 Simplification of the Near-Wake 


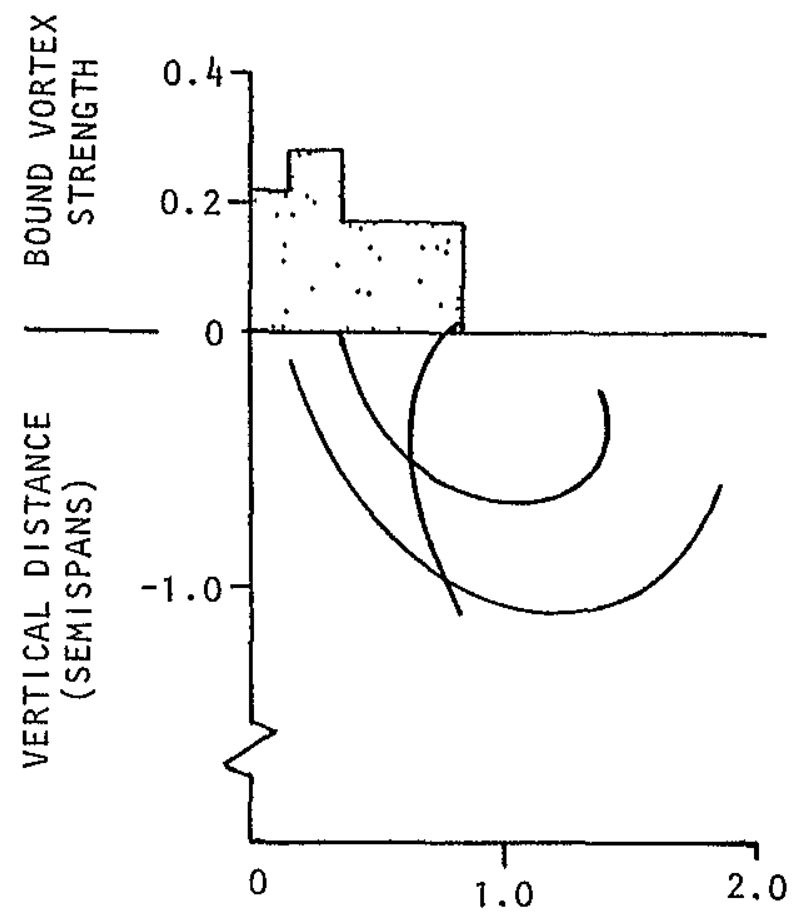

(a) "STANDARD" 2-D METHOD

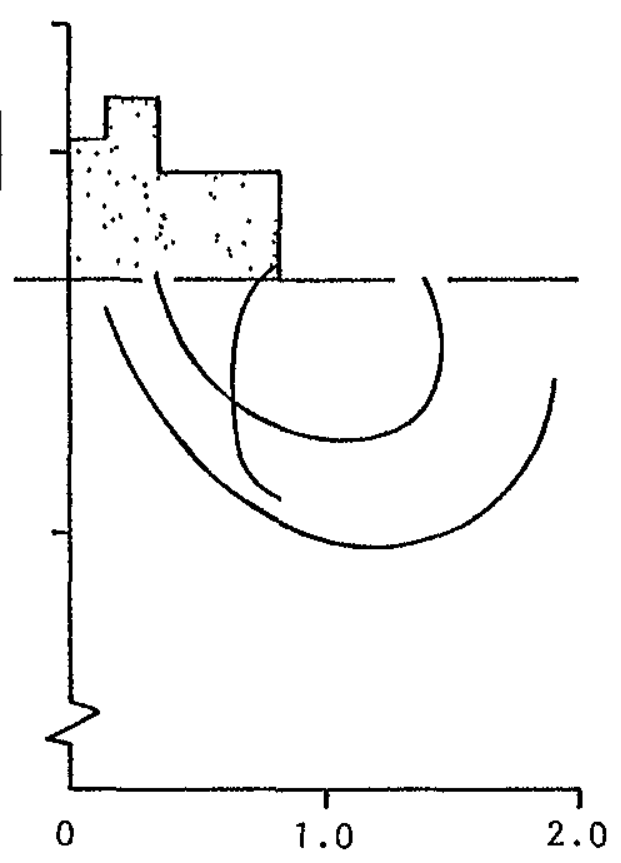

(b) HACKETT AND EVANS METHOD (REF. 9)

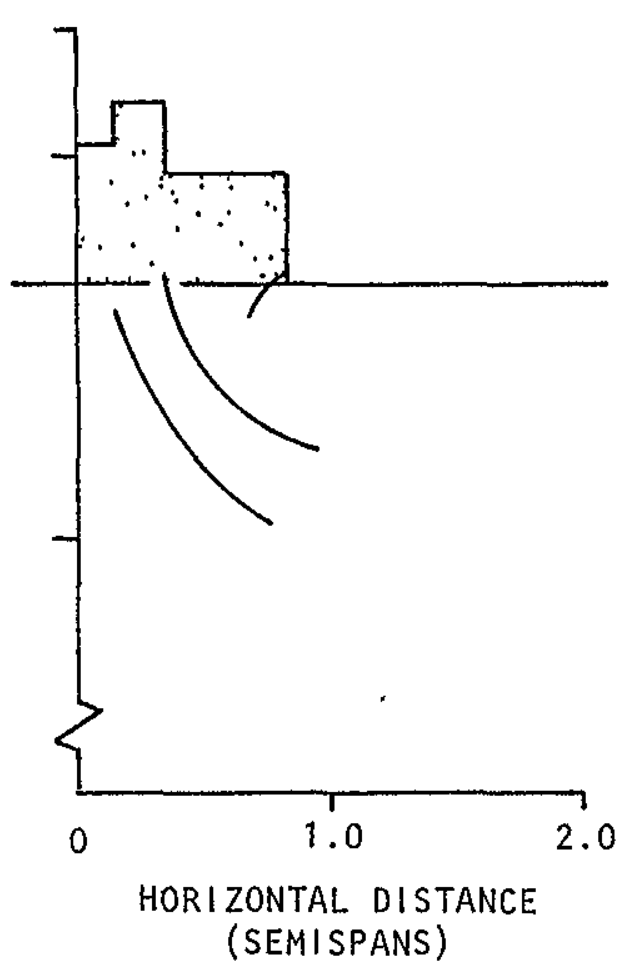

(c) 3-D RELAXATION

Figure 5.4 Calculation of the Initial Wake - Aircraft Fixed 

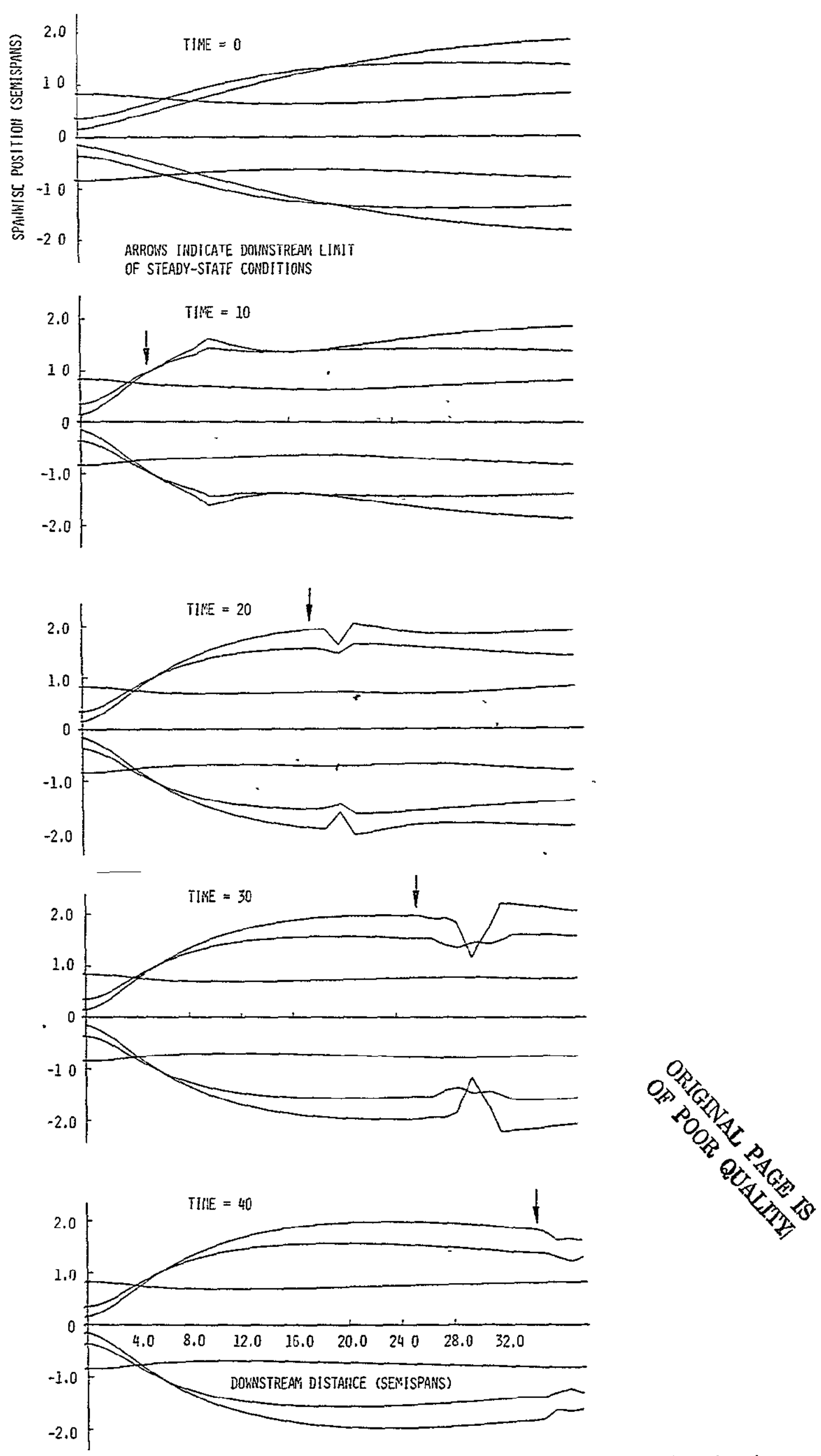

Figure 5.5 Three-Dimensional Relaxation to a Steady-State - Aircraft Fixed 


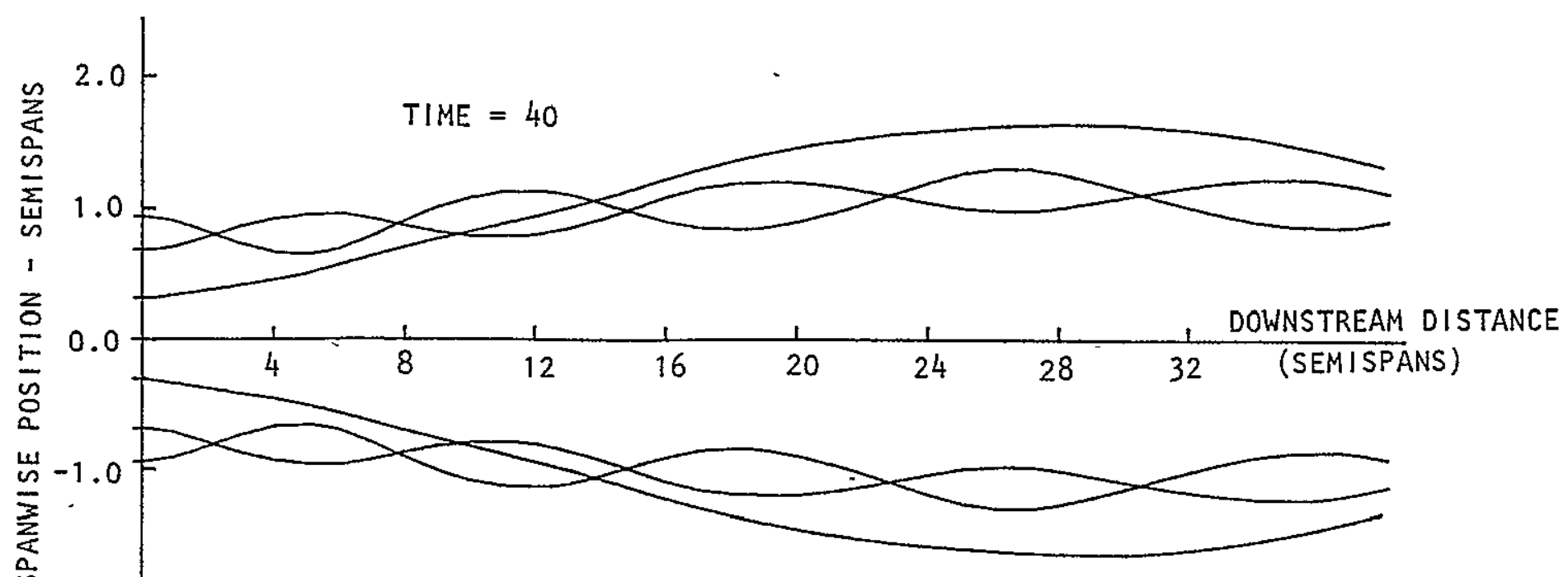

(a) Near-Cyclic Configuration

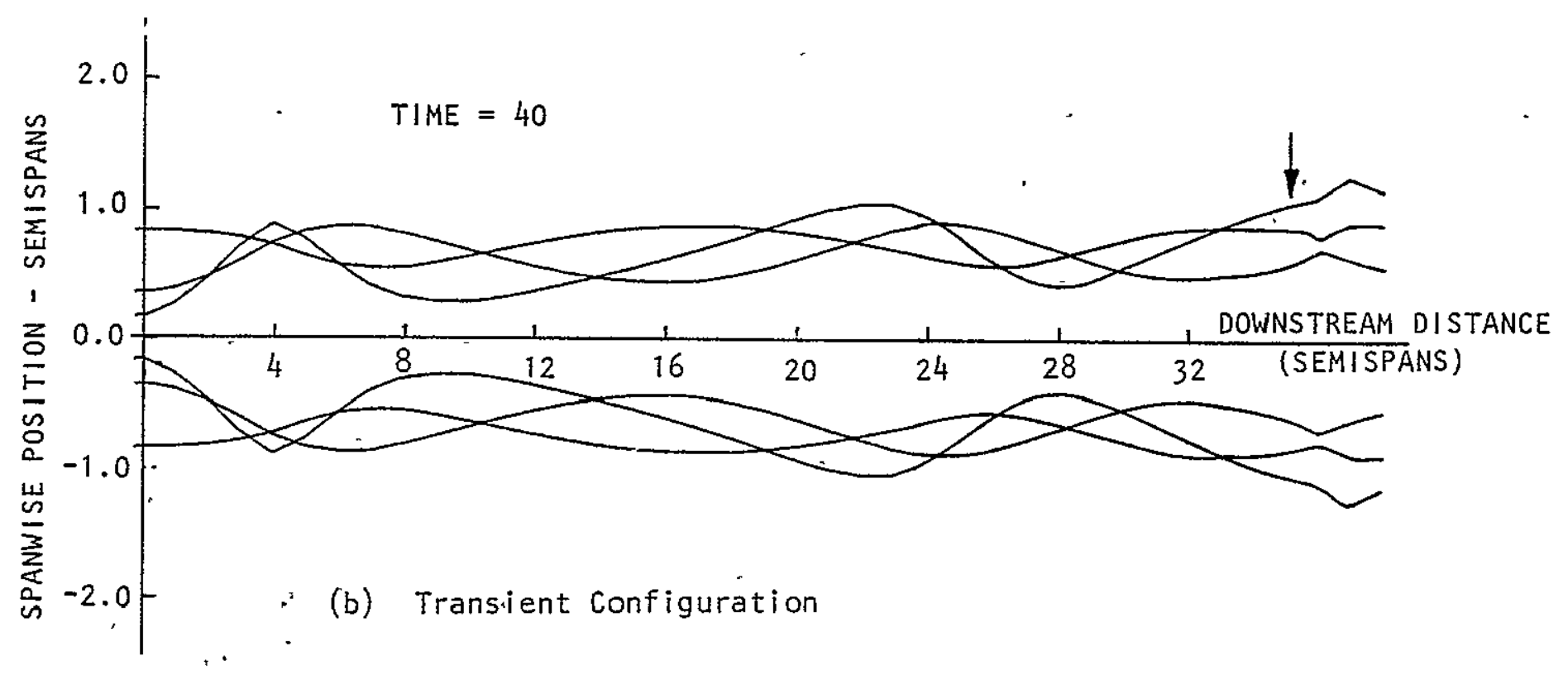

Figure 5.6 Near-Cyclic and Transient Cases 

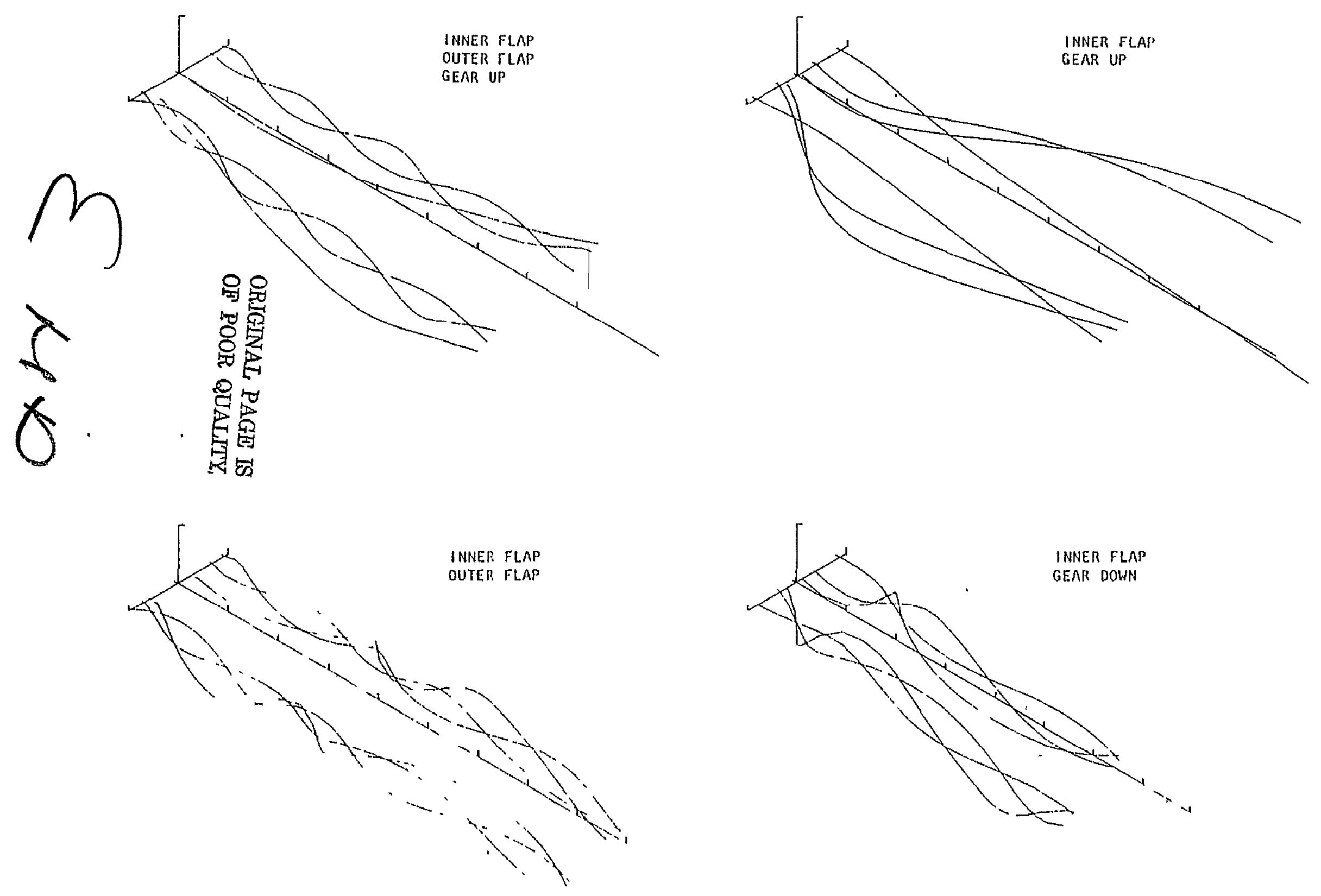

NOTC. "TICKS ON AXES RLPRESENT ONE SEMISPAN VERTICALLY AND SPANWISE AND ABOUT SIX SEMISPANS AXIALLYY 